\title{
HANDICAP IN STROKE SURVIVORS
}

by

\section{Philippa J. Clarke}

\author{
A thesis submitted in conformity with the requirements \\ for the degree of Master of Science \\ Graduate Department of Community Health \\ University of Toronto
}

C Copyright by Philippa Janet Clarke (1996) 
Acquisitions and Bibliographic Services

395 Wellington Street Ottawa ON K1A ONA Canada
Bibliothèque nationale du Canada

Acquisitions et services bibliographiques

395. ne Wollington

Ontawa ON K1A ONA

Canada
The author has granted a nonexclusive licence allowing the National Library of Canada to reproduce, loan, distribute or sell copies of this thesis in microform, paper or electronic formats.

The author retains ownership of the copyright in this thesis. Neither the thesis nor substantial extracts from it may be printed or otherwise reproduced without the author's permission.
L'auteur a accordé une licence non exclusive permettant à la Bibliothèque nationale du Canada de reproduire, prêter, distribuer ou vendre des copies de cette thèse sous la forme de microfiche/film, de reproduction sur papier ou sur format électronique.

L'auteur conserve la propriété du droit d'auteur qui protège cette thèse. $\mathrm{Ni}$ la thèse ni des extraits substantiels de celle-ci ne doivent être imprimés ou autrement reproduits sans son autorisation. 


\title{
HANDICAP IN STROKE SURVIVORS \\ Master of Science \\ 1996 \\ Philippa Janet Clarke \\ Graduate Department of Community Health \\ University of Toronto
}

\begin{abstract}
As the world's population ages and as acute disease and infections decline in prevalence, chronic conditions, such as stroke, have become predominant concerns. While stroke research has focused on outcomes such as mortality and morbidity, there is also a need to understand the impact of stroke on the meaningful lives of stroke survivors. Using the International Classification of Impairments, Disabilities and Handicaps (World Health Organization, 1980) as a framework, the purpose of this thesis was to investigate handicap in stroke survivors at three months and at one year after onset. The results indicate that stroke-related impairments and disabilities significantly compromise the meaningful lives of stroke survivors. Individuals who experienced motor disability and depression throughout the recovery period reported greater handicap than those who did not. Cognitive disability was a hindrance, but only at one year poststroke. Other factors, such as living arrangements, marital status, the use of rehabilitation therapy and a history of previous stroke were also influential. The findings indicate that an individual's life activities are threatened following stroke, and suggest possible resources that may be beneficial in moderating its impact.
\end{abstract}




\section{ACKNOWLEDGEMENTS}

The preparation of a Master's thesis takes a lot of energy and hard work, and although I feel that I have written this thesis in blood, I would surely not have survived this process without the help and encouragement of a number of individuals and organizations. I would first like to thank my thesis supervisor, Dr. J. Ivan Williams, for expertly, yet tenderly, guiding me through my "Baptism by Fire", such that I have emerged with only a few minor singes. The members of my thesis committee, Dr. Sandra Black and Dr. Elizabeth Badley, were invaluable in the sharing of their time, knowledge and insight. The generous financial support of the National Health Research and Development Program in the form of a M.Sc. Fellowship is gratefully acknowledged, as is the Heart and Stroke Foundation of Ontario, which funded the larger study upon which this work is based. The staff and resources at the Institute for Clinical Evaluative Sciences in Ontario and the Cognitive Neurology Laboratory at Sunnybrook Health Science Centre provided valued support and fellowship. I also thank Dr. John W. Norris for guiding me in the right direction in the first place. Additionally, I am ultimately indebted to the many stroke survivors who gave of their time and energy in this project. Finally, this project would not have been possible without the dedicated and thorough work of my colleague and friend, Joanne Lawrence, who meticulously collected and collated the data for this project.

On a more personal front, I consider myself blessed and fortunate to have a wonderful support network that has been invaluable to me throughout this thesis. I am grateful to my family and to Ninett Wasserman for their support and encouragement. Finally, to my mother, Aileen Clarke, and to my husband, Avi Wasserman, I am, as ever, sustained by your love, wisdom and inspiration. 


\section{TABLE OF CONTENTS}

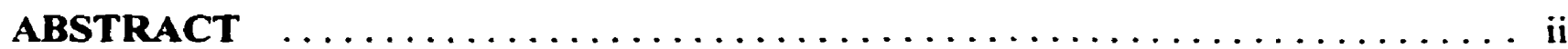

ACKNOWLEDGEMENTS $\ldots \ldots \ldots \ldots \ldots \ldots \ldots \ldots \ldots \ldots \ldots \ldots \ldots \ldots$ iii

TABLE OF CONTENTS $\ldots \ldots \ldots \ldots \ldots \ldots \ldots \ldots \ldots \ldots \ldots \ldots \ldots \ldots \ldots \ldots$ iv

INTRODUCTION $\ldots \ldots \ldots \ldots \ldots \ldots \ldots \ldots \ldots \ldots \ldots \ldots \ldots \ldots \ldots \ldots \ldots$

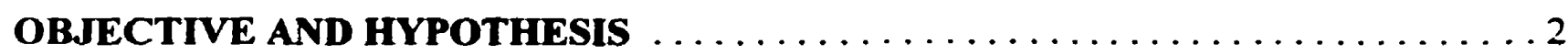

BACKGROUND AND RATIONALE $\ldots \ldots \ldots \ldots \ldots \ldots \ldots \ldots \ldots \ldots \ldots \ldots \ldots \ldots$

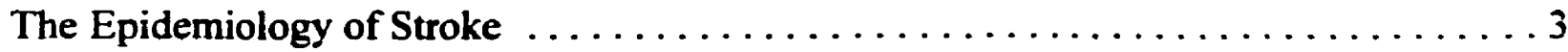

International Classification of Impairments, Disabilities and Handicaps $\ldots \ldots \ldots \ldots 6$

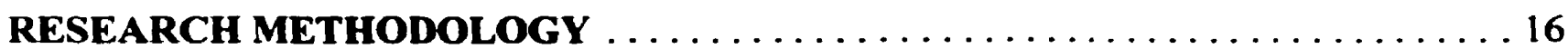

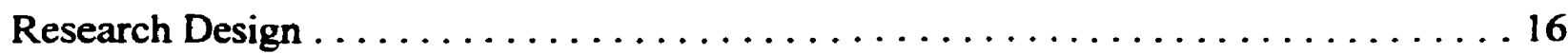

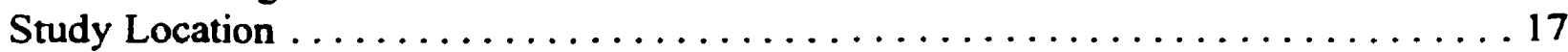

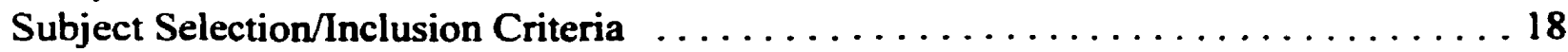

Instruments Used in the Collection of Information $\ldots \ldots \ldots \ldots \ldots \ldots \ldots \ldots \ldots$

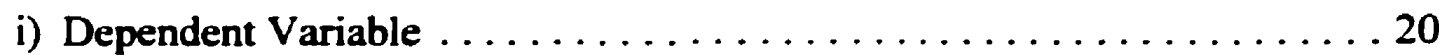

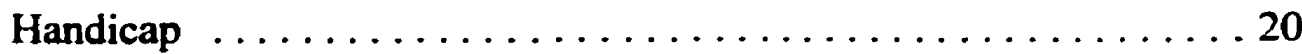

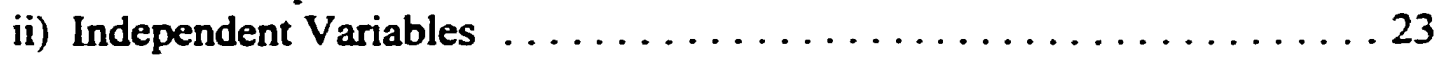

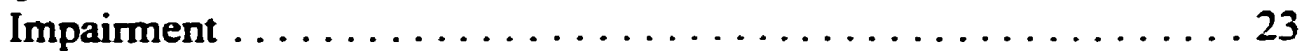

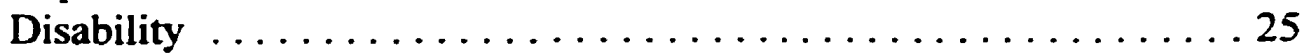

Environmental Factors . . . . . . . . . . . . . . 28

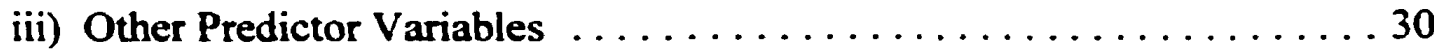

Procedures Used in the Collection of Information . . . . . . . . . . . . . . 34

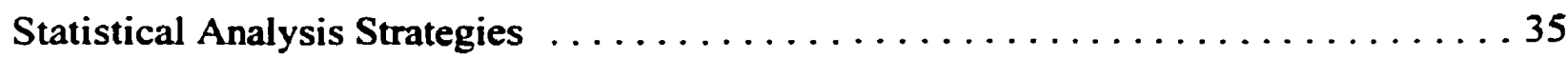

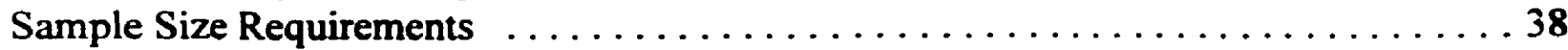

Ethical Considerations .................................... 39

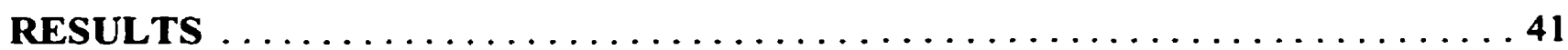

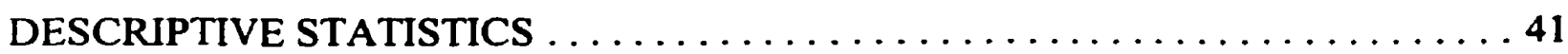

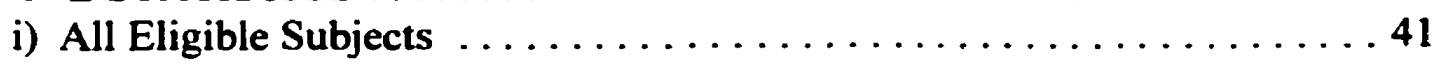

ii) Study Participants: Subjects Assessed For Handicap $\ldots \ldots \ldots \ldots \ldots 43$

Reasons for Non-Participation . . . . . . . . . . . . . . 43 


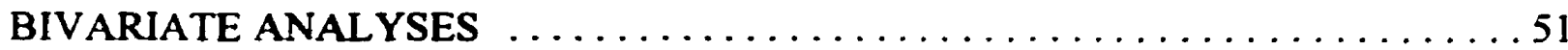

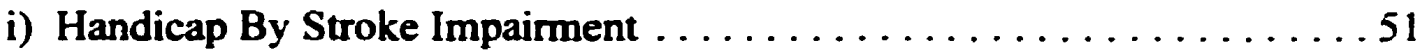

ii) Handicap by Stroke Disability . . . . . . . . . . . . . 53

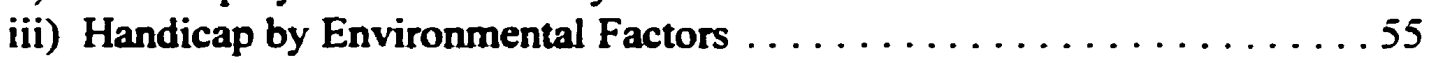

iv) Handicap by Pre-Stroke Disability $\ldots \ldots \ldots \ldots \ldots \ldots \ldots \ldots 7$

v) Handicap by Other Comorbid Conditions $\ldots \ldots \ldots \ldots \ldots \ldots \ldots$

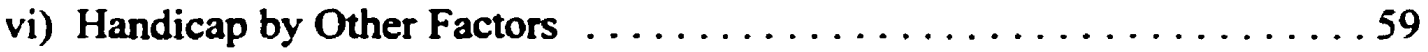

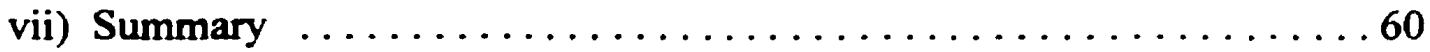

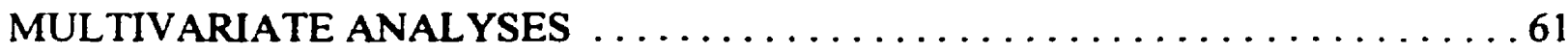

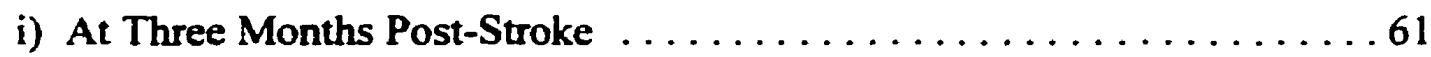

ii) At One Year Post Stroke .................. 67

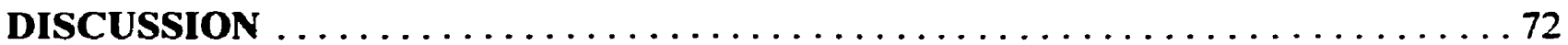

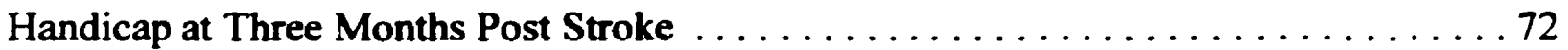

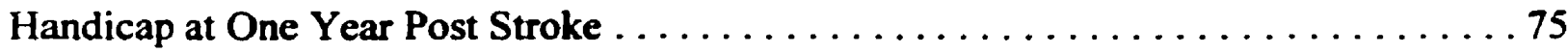

Summary . . . . . . . . . . . . . . . . . . . . . . . 79

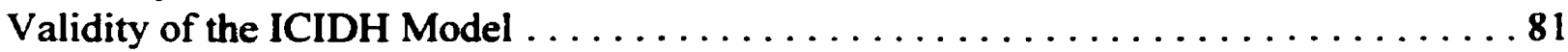

Limitations . . . . . . . . . . . . . . . . .

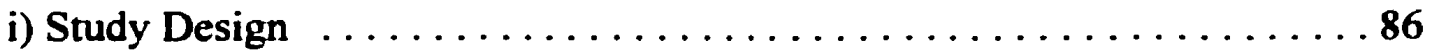

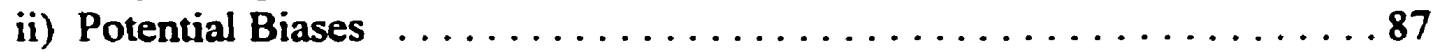

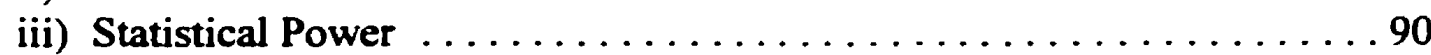

iv) Other Limitations $\ldots \ldots \ldots \ldots \ldots \ldots \ldots \ldots \ldots \ldots$

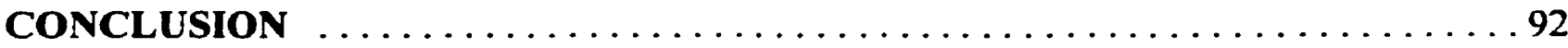

RECOMMENDATIONS FOR FUTURE RESEARCH $\ldots \ldots \ldots \ldots \ldots \ldots \ldots \ldots$

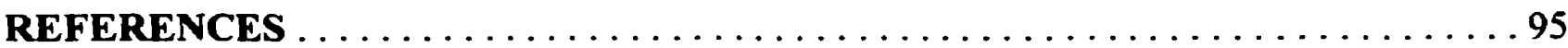




\section{LIST OF TABLES}

Table 1

Table 2

Table 3

Table 4

Table 5

Table 6

Table 7

Table 8

Table 9

Table 10

Table 11

Table 12

Table 13

Table 14
Average RNL Scores ( \pm s.d.) By Level of Impairment 52

Average RNL Scores ( \pm s.d.) By Depression $\ldots \ldots \ldots \ldots \ldots \ldots \ldots \ldots$

Average RNL Scores ( \pm s.d.) By Level of Disability 54

Average RNL Scores ( \pm s.d.) by Use of Rehabilitation Therapy . . . . . . . 56

Average RNL Scores ( \pm s.d.) By Level of Pre-Stroke Disability

Simple Linear Regression Between

Pre-Stroke Quality of Life and Post-Stroke Handicap .

Average RNL scores ( \pm s.d.) by Age Group $\ldots \ldots \ldots \ldots \ldots$

Multi-Way Analysis of Variance

Motor Ability and Cognitive Ability in Association with 3 Month RNL . . 62

Multi-Way Analysis of Variance

Motor Ability, Cognitive Ability and Depression

in Association with 3 Month RNL

Multi-Way Analysis of Variance

The Association of Motor Ability and Depression with 3 Month RNL

Multi-Way Analysis of Variance

Final Model Predicting 3 Month RNL

Average RNL Scores ( \pm s.d.)

by Rehabilitation Therapy and Motor Ability

Multi-Way Analysis of Variance

Motor Ability, Cognitive Ability and Depression

in Association with 1 Year RNL

Multi-Way Analysis of Variance

Motor Ability, Cognitive Ability, Depression, Stroke History,

Sex and Marital Status in Association with 1 Year RNL 69 
Table 15 Interaction Effect of Marital Status and Sex

Average RNL ( \pm s.d.) Scores by Marital Status and Sex

\section{LIST OF FIGURES}

Figure 1 Impairments, Disabilities and Handicaps as Outlined by the ICIDH $\ldots \ldots 7$

Figure $2 \quad$ The Handicaps Creation Process $\ldots \ldots \ldots \ldots \ldots \ldots \ldots \ldots \ldots \ldots \ldots$

Figure $3 \quad$ Reasons for No Handicap Assessment $\ldots \ldots \ldots \ldots \ldots \ldots \ldots \ldots$

Figure $4 \quad$ Effect of Disability on Handicap

According to the Use of Rehabilitation Therapy at Three Months. . . . . . . 82

Figure 5 Effect of Marital Status on Handicap

According to the Sex of the Subject at One Year . . . . . . . . . . 83

\section{LIST OF APPENDICES}

Appendix $1 \quad$ Description of Clinical Study $\ldots \ldots \ldots \ldots \ldots \ldots \ldots \ldots \ldots \ldots \ldots$

Appendix 2 Reintegration to Normal Living Index $\ldots \ldots \ldots \ldots \ldots \ldots \ldots \ldots$

Appendix $3 \quad$ Adams' Hemispheric Stroke Scale $\ldots \ldots \ldots \ldots \ldots \ldots \ldots \ldots \ldots \ldots \ldots$

Appendix $4 \quad$ Zung Self-rating Depression Scale $\ldots \ldots \ldots \ldots \ldots \ldots \ldots \ldots \ldots$

Appendix $5 \quad$ Functional Independence Measure $\ldots \ldots \ldots \ldots \ldots \ldots \ldots \ldots \ldots$

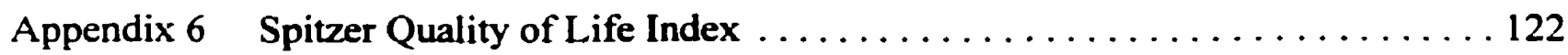

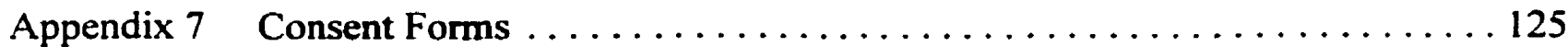

Appendix 8 Demographic Profile of All Eligible Subjects on Admission $\ldots \ldots \ldots$

Appendix 9 A Comparison of Participants and Non-participants $\ldots \ldots \ldots \ldots \ldots$ 
Ethics Approval

Permissions to Reproduce Copyright Material 


\section{INTRODUCTION}

The faces of medicine and health research have been changing over the past two decades in response to the need for a new perspective to replace the traditional biomedical model (Engel. 1977). As our population ages and as acute disease and infections decline in prevalence. chronic disease and disability are becoming predominant concerns. In response, there is a growing demand for more relevant health indicators, such as quality of life, to supplement the standard mortality and morbidity statistics.

Stroke is a disorder that bears witness to these demographic and medical changes. As stroke mortality rates decline and as the population ages, more and more people are living with the aftermath of a stroke, rather than immediately dying from it. There is, therefore, a pressing need to understand the impact of stroke on the lives of the growing number of individuals it affects. No longer is it sufficient to simply document survival and neurological impairment in stroke survivors. One must address the issues of return to valued activities in the home or community and the quality of life following onset of disease and its management.

Investigations seeking answers to these questions will facilitate a greater awareness of the social and personal impact of chronic illness such as stroke. The International Classification of Impairments, Disabilities and Handicaps (World Health Organization, 1980) was developed for just such a purpose, and this thesis makes use of this nomenclature, in particular handicap, to gain a better understanding of the origins and manifestations of the psychosocial sequelae of stroke. Only with this understanding can we possibly attempt to better the lives of the growing number of individuals in our society today who suffer from chronic disease and disability. 


\section{OBJECTIVE AND HYPOTHESIS}

The objective of this thesis is to investigate handicap in stroke survivors using the framework of the World Health Organization's International Classification of Impaiments Disabilities and Handicaps (ICIDH) (World Health Organization, 1980). Handicap is defined as "a disadvantage for a given individual that limits or prevents the fulfilment of a role that is normal (depending on age, sex, social and cultural factors) for that individual"(World Health Organization, 1980). The purpose of this thesis is to determine the extent to which stroke induced impairments and disabilities precipitate handicap following stroke. It is hypothesized that individuals with more extensive impairments and disabilities will experience greater handicap, although it is recognized that not all of the variance in handicap will be explained by these factors. 


\section{BACKGROUND AND RATIONALE}

\section{The Epidemiology of Stroke}

Approximately 50,000 Canadians suffer a stroke each year (Heart and Stroke Foundation of Ontario, 1994; Petrasovits \& Nair, 1994), with incidence rates estimated to range between 1.4 to 2.3 per 1,000 individuals (Bonita \& Beaglehole, 1995; Gordon, 1993). Stroke is more common in those people with hypertension, diabetes and cardiac dysrhythmias; smoking, obesity and inactivity also elevate the risk. But age is the biggest risk factor. Individuals over the age of 75 have been found to be at five times the risk for stroke than those between the ages of 55 and 64 (Oxfordshire Community Stroke Project, 1983). Stroke is, therefore, most common in Canadian seniors, with incidence rates doubling for each decade of life after the age of 55 (Gordon, 1993). As the Canadian population ages, more and more people will be in these higher age groups, increasing the number of individuals at risk for stroke. By the year 2036, Canadians over the age of 65 will make up twice as much of the population as they do now, constituting one quarter of the total population (Desjardin, 1992); half of this group will be over 75 years of age.

While stroke is currently the third most common cause of death in the industrialized world after heart disease and cancer (Bonita, 1992), mortality rates have been declining up to a rate of about 7\% per year (Bonita \& Beaglehole, 1995; Bonita, 1992; Wolf et al., 1992). Canada now has one of the lowest rates of stroke mortality in the world (Bonita, 1992). The reasons for this decline are uncertain, but may be due to an increased likelihood of survival (Shahar et al., 1995), or to the decline in stroke incidence as a result of improved detection and treatment of hypertension (Bonita \& Beaglehole, 1993). Nevertheless, these declining mortality trends coupled with the simultaneous growth in the aging population have created a pool of stroke survivors with varying degrees of physical and cognitive disability. Therefore, even though the incidence of stroke may have been declining, the prevalence of stroke has remained constant (Millikan, McDowell \& Easton, 1987b). Prevalence estimates range from 6-8 per 1,000 for those over 25 years of age, and can be has high as 54 per 1,000 for those over the age of 65 (Gordon, 1993). In fact, it is projected that with the increase in 
the number of elderly people, stroke incidence will actually increase by $22 \%$ by the year 2016 (La Rosa et al., 1993).

Stroke accounts for one of the greatest number of days of hospital care in Canada, with 1.5 million days of hospital stay for males and 1.9 million days for females reported between 1989 and 1990 (Statistics Canada, 1992), almost half of which were used in Ontario. Health Canada anticipates that by the year $\mathbf{2 0 0 0}$ the number of hospital days due to stroke in Canadian seniors will almost double from their current level (Desmeules, Huang, \& Mao, 1993). In elderly women stroke will be responsible for the greatest number of days of hospital stay, after accidental falls and ill-defined symptoms. It is estimated that both the direct and indirect costs of stroke in Canada reach $\$ 1.5$ billion per year (Veloso, 1994).

Stroke occurs as a result of a disruption of blood supply to the brain, causing impaired bodily function as brain cells in the affected parts of the brain die from lack of oxygen. There are two main types of stroke: cerebral infarction and cerebral hemorrhage, both resulting from a disruption in cerebral blood flow, but by two different means. In a cerebral infarction, the blood supply to the brain is restricted by a blockage in one of the cerebral arteries, while cerebral hemorrhage occurs when the blood supply is restricted because of a burst artery. Infarction is the most common of the two, responsible for about $90 \%$ of all completed strokes (Chambers, Norris, Shurvel, \& Hachinski, 1987), and the arterial blockage is usually caused by diseased blood vessels (atherosclerosis) or a diseased heart. Atherosclerosis occurs from fat deposits in the arterial walls that start to develop in childhood and eventually accumulate at various rates to significantly compromise cerebral circulation in later life. These fatty deposits grow through scarring and clot formation, and may either cause infarction by restricting the blood flow in vessels leading to the brain, or by releasing clot material into the blood stream where it eventually blocks a smaller arterial passage. Similarly, a diseased heart may cause an infarction if cerebral blood flow is reduced by cardiac failure, or if cardiogenic clots are released into the blood stream.

Most of the damage caused by a hemorrhage comes not from the lost blood supply from the burst artery (since they tend to occur in the small arteries deep in the brain), but from the resulting cell death as brain tissue is confined by the pooling blood. Hypertension is the 
suspected cause of most of these ruptures, as it is thought to weaken the arterial structure. Hemorrhages are more likely to be fatal than infarctions (Chambers, Norris. Shurvel, \& Hachinski, 1987), with $100 \%$ mortality in larger bleeds (over $3 \mathrm{~cm}$ in diameter).

Unfortunately, current medical practice has little in the way of effective treatment for a completed stroke (Ebrahim, 1990). Once an event has occurred, little can be done to reverse the damage. and survivors must live with the resulting consequences of vessel damage. Because the brain is such a dynamic and complex organ, the body systems that are compromised by a stroke depend on the areas of the brain that are affected. The brain is divided into two hemispheres (left and right) and the brain stem (supplied by the vertebrobasilar arteries). Each hemisphere has four lobes (frontal, temporal, parietal and occipital), each responsible for a different aspect of human function. If the damage occurs in the area between the frontal and parietal lobes of the left hemisphere for instance, then motor and sensory problems occur on the right side of the body. If the stroke occurs in the occipital lobe, then visual impairments such as blindness usually occur, while language is affected by lesions in the tempo-parietal regions. Strokes in the vertebrobasilar system often result in dizziness and nausea.

Therefore, the disabling effects of stroke may be devastating and can encompass many facets of everyday life. Depending on the affected area of the brain, an individual who experiences a stroke may suddenly be left with paralysis or weakness in the upper and/or lower extremities, incontinence, visual impairment, loss of sensation in or awareness of one side of the body, difficulty in swallowing and difficulty in understanding what is said and in communicating with others. Individuals may also become emotionally labile after a stroke, with sudden outbursts of crying or rage for no apparent reason. Personality changes may also occur: individuals with strokes in the left hemisphere of the brain may adopt a slow, cautious behavioural style, while those with lesions in the right hemisphere can become uncharacteristically impulsive. Roughly half of those people with left brain damage have an impaired ability to comprehend or express language (aphasia) (Kertesz \& Black, 1985), while spatial and perceptual problems, such as hemi-spatial neglect, occur in $50-80 \%$ of individuals with right brain damage (Hier, 1983). Unfortunately, these impairments tend to be long lasting, remaining with many stroke survivors for the rest of their lives. Approximately $40 \%$ of stroke 
survivors are still dependent in self care at one year post-stroke (Aho, Harmsen, Hatano. \& et al. 1980; Andrews, Brocklehurst, Richards, \& Laycock, 1981), and $75 \%$ never recover full independent motor function (Gowland, 1982; Partridge, Johnston, \& Edwards, 1987). particularly in the upper extremities (Wade, Hewer, \& Wood, 1983).

The extensive and often permanent nature of these impairments can compromise an individual's quality of life. Survivors often experience impaired family relations (Brocklehurst, Morris, \& Andrews, 1981), sexual dysfunction (Bray, DeFrank, \& Wolfe, 1981) and insomnia (Doolittle, 1991) after a stroke. Furthermore, $61 \%$ of women and $87 \%$ of men report a restriction in their usual activity following stroke (Verbrugge, 1984), particularly in social and environmental activities, such as vocational function and socialization outside the home (Gresham, Philips, \& Wolf, 1979). One study found that only half of previously employed stroke victims had returned to work by one year after their stroke (Kotila, Waltimo, \& Marjaiisa, 1984).

Therefore, the psycho-social consequences of a stroke can be substantial. Survivors often feel they are no longer "normal", using such words as "cripple", "defective" and "useless" to describe themselves (Becker, 1993). Yet, the emphasis of stroke outcome research remains focused on the physical and self care aspects of recovery with little attention paid to issues such as quality of life and handicap (de Haan, Aaronson, Limburg, Langton Hewer, \& van Crevel, 1993; Seale \& Davies, 1987). Research into the impact of stroke on the day to day lives of individuals has generally focused on limitations in mobility and activities of daily living (Seale \& Davies, 1987) in spite of the fact that physical disability has been shown to be only a minor part of the experience of stroke survivors (Becker, 1993; Gresham, 1986; Gresham et al., 1975; Labi, Phillips, \& Gresham, 1980). It is therefore necessary to begin to examine the social and psychological consequences of stroke in order to increase our understanding of this common neurological disorder.

\section{International Classification of Impairments, Disabilities and Handicaps}

The International Classification of Impairments, Disabilities and Handicaps (ICIDH) (World Health Organization, 1980) can provide a useful conceptual framework to explore the psycho- 
sociological impact of stroke. In fact, it has been advocated that the ICIDH is the best way to approach any epidemiological study of stroke (Granger \& Gresham, 1990). This classification system was developed to complement the International Classification of Diseases (ICD) (World Health Organization, 1977) which catalogues disease using the concept of diagnosis. Although the ICD satisfies the needs of the curative medical model, it is limited in its application to the experiences of individuals once the acute stage of the disease has passed. As chronic disease becomes more prevalent, it is evident that the concept of diagnosis is simply not adequate to explain the consequences of ongoing disorders. Therefore, in response to these demands, the World Health Organization (WHO) published the ICIDH in 1980 as a conceptual scheme to highlight the long term consequences of more chronic diseases and injuries. Since its publication, this manual of classification has become increasingly popular throughout the world, with translations in 14 languages and over 1000 articles published on its use (World Health Organization, 1993). Indeed, there has been such a demand for the ICIDH, that a second printing was necessary in 1993.

The ICIDH first identifies impairments as the consequence of disease at the level of the body or organ (Figure 1). Impairments represent any loss or abnormality in the psychological, physiological or anatomical structure or function of the body. For the stroke victim the

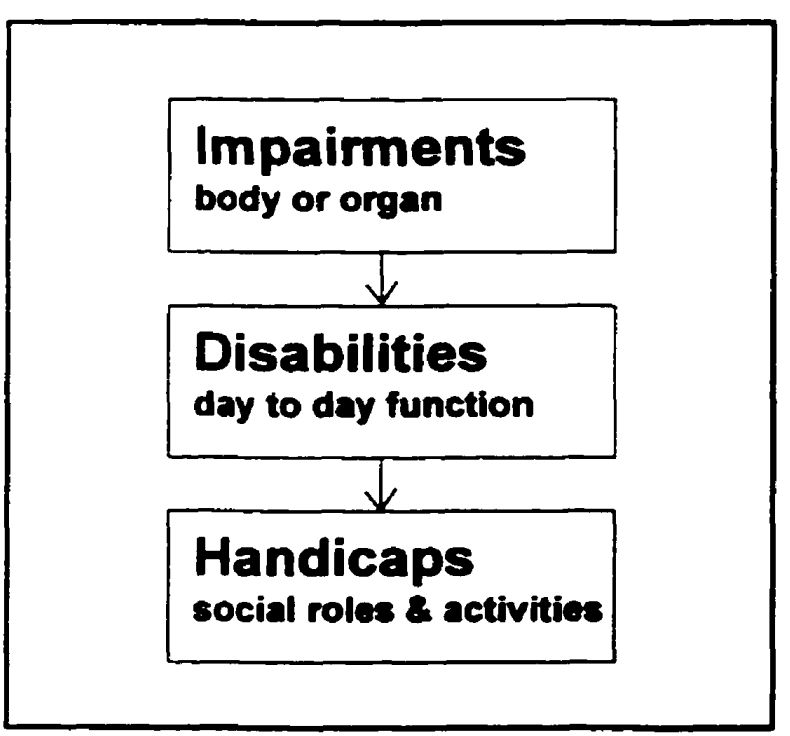

Figure 1 Impairments Disabilities and Handicaps as outlined by the ICIDH damage to the brain and the nervous system can result in physical and cognitive impairments, such as a weak leg. These impairments, in turn, can lead to disabilities which are consequences of disease at the level of the person's day to day function and represent restrictions in the ability to perform activities in the manner or range considered normal for a human being. The physical and mental limitations in speech, mobility, movement and cognition are some of the disabilities that can result following a stroke. For example, a stroke survivor with a weak leg 
may experience a disability in walking or climbing stairs. By emphasizing activities, disability is concerned only with what takes place, not with the meaning attached to it.

Finally, these impairments and disabilities can then lead to handicaps, which are the consequences of disease at the social level, and include the inability to participate fully in social, recreational or vocational activities. Handicap is concerned with the value individuals attach to their situation following an illness. It is, therefore, subjective and to a certain extent dependent on cultural norms. Six key dimensions of handicap have been identified by the WHO (World Health Organization, 1980) as fundamental to the social existence of human beings across cultures:

1) Orientation: the ability of individuals to orient themselves in regard to their surroundings and respond to these inputs;

2) Physical Independence: the ability of individuals to maintain an effective independent existence in regard to the more immediate needs of their bodies, including feeding and personal hygiene;

3) Mobility: the ability of individuals to move around effectively in their environment;

4) Occupation: the ability of individuals to occupy time in a fashion customary to their age, sex and culture, including following an occupation (such as tilling the soil, running a household or bringing up children) or carrying out physical activities such as play and recreation;

5) Social Interaction: the ability of individuals to participate in and maintain social relationships with others;

6) Economic Self-Sufficiency: the ability of individuals to sustain socioeconomic activity and independence by virtue of labour or exploitation of material possessions such as natural resources, livestock or crops.

Although many of these dimensions may be perceived as relating to disability (particularly the first three), it is important to recognize the distinction between disability and handicap. Because handicap is a social phenomenon, these dimensions are not concerned with the ability to perform the activity itself, but rather the meaning of the inability to perform these activities from the perspective of the individual in the social realm. For instance, a stroke survivor with a weak leg who has a disability in walking and climbing stairs may find it difficult to get around and participate fully in work, recreational and social activities. This person would then experience the consequences of disability in the mobility, occupation and social integration dimensions of handicap. To illustrate this point further, consider a similar stroke 
survivor who also has a disability in walking and climbing stairs, but who has the assistance of a motorized wheelchair. Although this person still has a disability, the mechanical device may permit the continued participation in occupational and social activities to a satisfactory extent. Handicap would then be reduced even though there has been no change in the disability.

Handicap, therefore, is a situational construct which represents the consequences of disabilities in the social context, not the disability itself. An examination of handicap after stroke can, therefore, shed some light on the meaning of stroke in the social context, and by situating handicap within the conceptual framework of the ICIDH, it is possible to examine the extent to which impairments and disabilities following stroke impact on the psychosociological lives of individuals. However, it must be kept in mind that the ICIDH is a classification tool only, not a description of persons. Handicap is a situational definition, not a description of the characteristics of an individual. And while the word handicap may have negative undertones in its common usage, that is not the intention of its use in this framework.

There has been considerable discussion about the conceptual relationships between impairment, disability and handicap, which has been well reviewed by Badley (1995). While the model in Figure 1 indicates that impairments lead to disabilities which then lead to handicaps, this sequential pathway may not necessarily be the case. In the first place, impairments may lead directly to handicap without the intervening presence of disability. Consider the case of a stroke survivor with residual facial weakness resulting in a left facial droop. This impairment would not likely lead to a disability since that person could continue to function independently in all day to day activities, such as walking and self care. However, the facial disfigurement may be a source of embarrassment for the individual, resulting in a reluctance to socialize with friends or be seen in the public world. Therefore, this physical impairment may create a handicap situation for this individual without the intervening presence of a disability.

Secondly, impairments and disabilities may not necessarily lead to handicap. For a stroke survivor with a weak leg, the resulting disability would create a substantial occupational handicap for someone who worked as a manual labourer, but may not create such a handicap 
for someone who worked as a radio announcer. But on the other hand, a speech disability, such as an expressive aphasia, would be a severe handicap for a radio announcer, but perhaps not so catastrophic for a manual labourer.

Implicit in these scenarios is the underlying importance of external forces in the individual's surrounding environment which may be influential in the genesis of handicap. Environmental factors may be physical, such as geographic location, architectural or structural factors; social, such as cultural attitudes and values; or, pertain to the availability of resources, such as mechanical aids, social supports and the use of health services (including rehabilitation therapy). Therefore, a number of models have been proposed which emphasize the importance of environmental factors in the conceptual scheme of the ICIDH (see review in Badley, 1995). As the ICIDH undergoes a revision in the next five years, one of the challenges will be to incorporate environmental factors as outlined by these models. These models range from the extreme, where environmental factors alone are seen to be responsible for handicap situations in those with impairments, to less radical models which more closely resemble the basic model of the ICIDH. While no particular model has gained overall acceptance, they all recognize that handicap may vary depending on the environment through which individuals pass.

To illustrate the potential role of environmental factors, Figure 2 depicts one of the models which was developed by the Canadian Society for the ICIDH (CSICIDH) (Canadian Society for the ICIDH, 1991). One can see that this model incorporates the main components of the ICIDH model, but emphasizes the importance of environmental factors in the handicaps creation process. According to this model, handicap is seen to be a situational result of an interactive process between impairments, disabilities and environmental factors. That is to say, the effects of impairments and disabilities on handicap may vary according to the presence or absence of an environmental factor. The small circle at the intersection of the lines in the model indicates the presence of this interaction.

Although there is a general consensus that environmental factors affect the extent to which impairments and disabilities create handicap, a number of theoretical interpretations exist concerning the nature of these factors. While the CSICIDH model depicts environmental 


\section{HANDICAPS CREATION PROCESS}

FIGURE 2 (with Permission from the Canadian Society for the ICIDH)

\section{RISK}

FACTORS

\section{CAUSES}

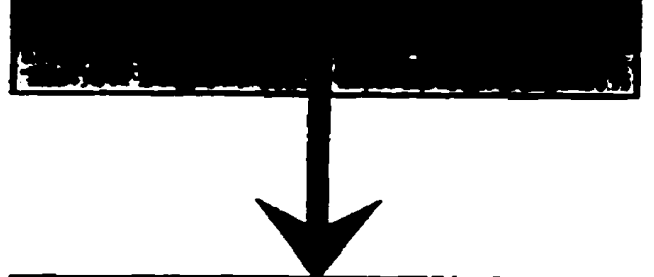

ORGANIC SYSTEMS

ABILITIES

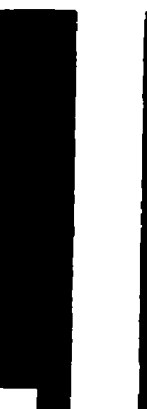

IMPAIRMENTS

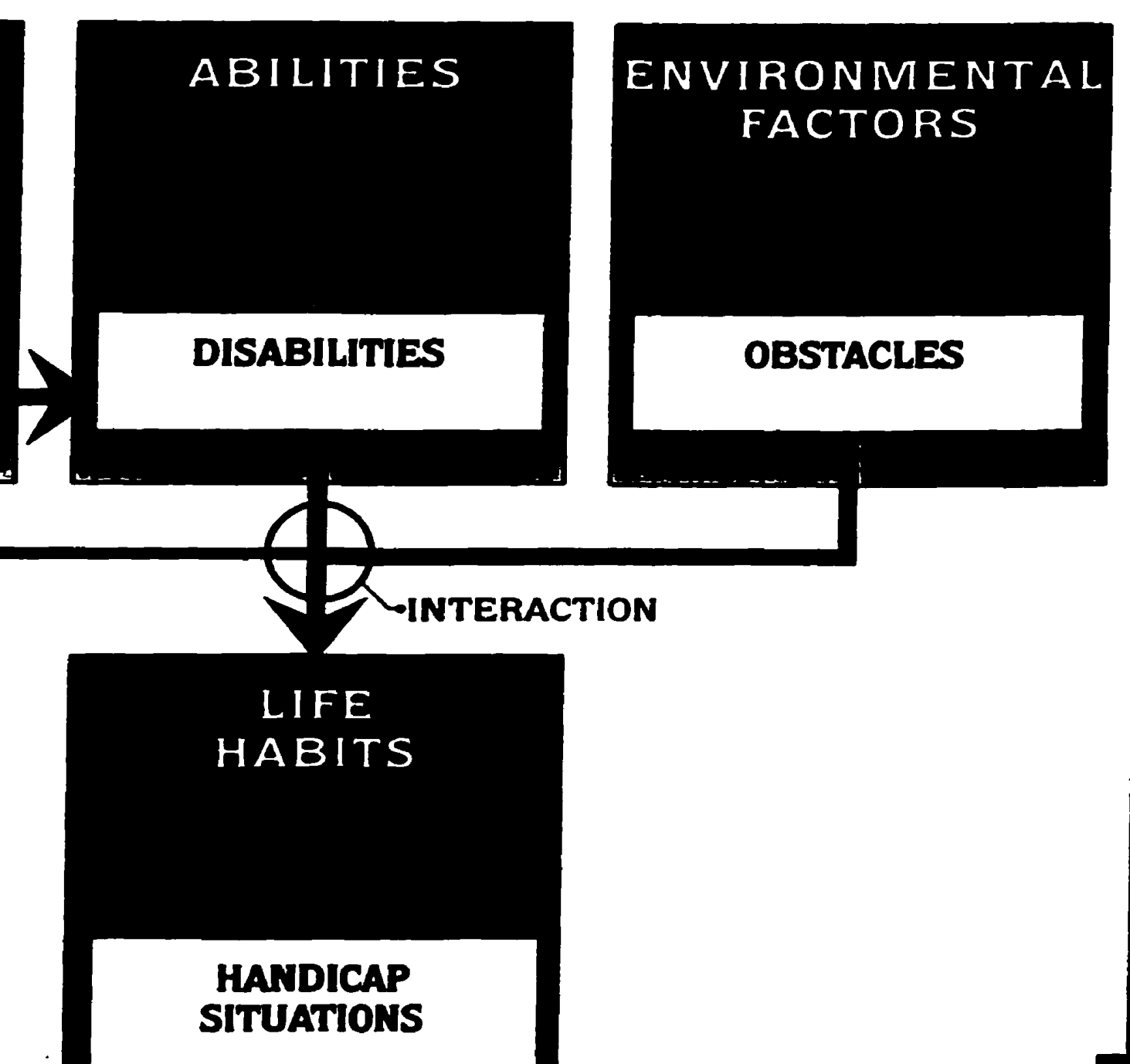

CSICIDH. 1991 
factors as "obstacles", suggesting that they are detrimental to handicap, they can also act in a positive way to mitigate the effects of impairment and disability on handicap. Authors such as Chamie (Chamie, 1989) have, therefore, divided environmental factors into those that have a positive effect on handicap (health and social services, social supports) and those that have a negative or exacerbating effect (architectural barriers, negative cultural attitudes, poverty). On the other hand, Verbrugge and Jette (Verbrugge \& Jette, 1993) have chosen to conceptualize environmental factors as either extra-individual, existing outside the individual, or intra-individual, indicating resources intrinsic to the individual, such as coping strategies, psychological outlook and behaviour. Furthermore, Badley (1995) has argued that environmental factors can be conceptualized as either pre-existing or responsive. Pre-existing factors are present in the person's surrounding environment independently of impairment and disability (such as architectural structures, education, wealth, living arrangements), while responsive factors only become active in the presence of impairments and disabilities (such as the use of aids and rehabilitation services). Additionally, McDonough, Badley and Tennant (1995) identify environmental factors as either resources that are mobilized to reduce the impact of impairment and disability on handicap, or social role obligations (family or work) that may act as motivating factors to reduce the impact of impairment and disability on handicap.

There is, therefore, considerable discussion about the nature of external factors which needs to be further explored in disablement research. But perhaps more importantly, there is a pressing need to understand the mechanisms through which these external forces affect handicap. While models such as the one from the CSICIDH explicitly indicate that an interaction takes place between impairments, disabilities and environmental factors, specific interaction effects are rarely included in the empirical body of disablement research. The nature of a statistical interaction indicates that the effect of impairments and disabilities on handicap will be different according to the presence or absence of an environmental factor. For instance, the effect of disability on handicap may be more pronounced in those without social supports. Therefore, research using the interactive models of the ICIDH should include explicit discussion of model pathways and specific testing of interaction effects. However, 
most of the research performed to date has only examined the independent effects of external factors, neglecting to specify their role in the genesis of handicap or include their interaction effects as postulated by these theoretical models.

An exception to this trend is the recently published work by McDonough, Badley and Tennant (1995) that systematically examined the effects of environmental factors on mobility handicap using statistical interactions with linear modelling. They found that the effects of walking disability on mobility handicap varied according to the presence of a car. For people with only minimal disabilities, there was little difference in the handicap reported for those with and without a car. But for people with a more severe walking disability, having a car reduced the impact of disability on mobility handicap (McDonough, Badley, \& Tennant, 1995). This effect was detected using a statistical interaction term between disability and car ownership. Similar research needs to be done examining the explicit nature of the interactive effects of environmental factors on handicap in order to better understand the mechanisms through which handicap is created. Only with this knowledge can we attempt to mediate the effects of impairment and disability in the lives of individuals.

The importance of the ICIDH in stroke outcome research was recognized over six years ago at a North American Symposium designed to mount a more effective research effort with which to alleviate the burden of disability and handicap in stroke survivors (Gresham, 1990). The ICIDH was identified at this Symposium as "the best way to organize any approach to a problem in the clinical epidemiology of disabling chronic disease, such as stroke outcome research" (Granger \& Gresham, 1990). Yet, the Symposium Task Force on stroke handicap concluded that research into the impairments and disabilities following stroke is far more advanced than that of stroke handicap, and "the overwhelming conclusion is that the area of handicap needs to be vigorously addressed by further conceptual and research efforts in ... stroke outcome research" (Task Force on Stroke Handicap, 1990).

Nevertheless, in spite of these recommendations, research in stroke handicap over the past six years has been scarce. A small number of studies have investigated quality of life following stroke. For instance, in two prospective studies of stroke survivors, depression (Sugisawa, 1991), disability, personality traits, disease awareness and marital status (Johansson et al., 1992) 
were found to be important factors influencing quality of life following stroke. But while these studies have been useful in raising awareness of more psycho-sociological outcomes. quality of life is so broadly defined (often including impairments, disabilities and handicaps along with subjective well-being (de Haan, Aaronson, Limburg, Langton Hewer, \& van Crevel. 1993)) that handicap is not the specific outcome of interest. The loose nature of this outcome only serves to further muddy the waters in an already murky pond.

Furthermore, these studies have been plagued by a number of methodological shortcomings. including the use of crude measurement scales without any demonstrated reliability and validity, and the inclusion of non-stroke patients in the population under study. For instance, Ahlsio and colleagues (Ahlsio, Britton, Murray, \& Theorell, 1984) conducted a prospective assessment of quality of life in $\mathbf{9 6}$ stroke patients at one, six and $\mathbf{4 8}$ months after onset and found that as disability increased, quality of life decreased. However, their measure of disability was limited to activities of daily living, and $22 \%$ of their cohort consisted of subjects with TIAs, which differ in outcome to stroke. Hayashi et al. (Hayashi, Ahiko, \& Yasumura, 1995) found that contact with friends was the most important determinant of quality of life for those stroke survivors under 65 years of age, while competence in eating was more important for those over the age of 65. However, quality of life was assessed with a crude visual analogue scale of global life satisfaction with unestablished psychometric properties. A recent study published by de Haan and colleagues (de Haan, Limburg, Van der Meulen, Jacobs, \& Aaronson, 1995) prospectively examined quality of life in stroke survivors six months after onset, but limited their analysis to the effects of lesion location and hemisphere on quality of life.

Even the few studies that have attempted to examine handicap following stroke within the ICIDH framework have been limited. Perry et al. (Perry, Garrett, Gronley, \& Mulroy, 1995) undertook a sound empirical examination of the impact of impairments and disabilities on handicap following stroke, but limited their analysis to mobility handicap only. Similarly, while Soderback and Ekholm (Soderback \& Ekholm, 1992) used the ICIDH framework in their work, the results were obscured by the inclusion of subjects with brain trauma as well as stroke.

Therefore, in spite of the call for specific research in the field of stroke handicap, few studies have specifically examined handicap within the theoretical framework of the ICIDH, as 
advocated by the Symposium on Methodological Issues in Stroke Outcome Research. The purpose of this thesis is to attempt to address this deficiency using the framework of the ICIDH to investigate the effects of stroke on the meaningful life of the individual. This thesis will examine the extent to which stroke induced impairments and disabilities precipitate handicap following stroke with specific attention paid to the interaction effects of environmental factors. 


\section{RESEARCH METHODOLOGY}

\section{Research Design}

The thesis question was investigated using data collected by Dr. Sandra Black and colleagues in a prospective cohort study of consecutive hemispheric stroke patients admitted to Sunnybrook Health Science Centre in Toronto (SHSC) (see detailed description of study in Appendix 1). The general objective of the study was to correlate measures of physical and neurocognitive deficits associated with hemispheric stroke with lesion parameters obtained from brain imaging techniques in order to better understand the effects of these anatomical findings on clinical deficit and recovery. While the primary objective of the study had a clinical focus, a secondary objective was to assess the impact of stroke on day to day social functioning. Therefore, this M.Sc. thesis project falls within the secondary objective of the study and represents the first analysis of the data in this respect, giving the student substantial independence in the analysis and presentation of results.

The study was conducted over a four year period from August 1990 to July 1994. Subjects were recruited from consecutive stroke patients admitted to Sunnybrook Health Science Centre between August 1, 1990 and May 31, 1993, with the exclusion of the months of September 1991 and May, June July and August 1992, when subjects were not accrued in order to allow the investigators to catch up on data collection. The random nature of these missing months makes it unlikely that any biases in subject selection would result. They occurred randomly, driven by the availability of resources, and it is highly unlikely that the characteristics of the final study population would be any different if data collection had continued throughout all months. Study end-points were one year completed follow-up, death or re-stroke, in which case the subject was re-entered into the study as a new case.

All consenting subjects were interviewed at three and twelve months following stroke onset. These two follow-up time periods were specifically chosen in order to assess the impact of stroke in the two clinically important periods of stroke recovery. Most of the spontaneous recovery in self care ability, language and motor ability occurs in the first three months after stroke (Andrews, Brocklehurst, Richards, \& Laycock, 1981; Partridge, Johnston, \& Edwards, 1987; Skilbeck, Wade, Hewer, \& Wood, 1983), with some additional improvement up to six months, 
and little change after this. Therefore, an assessment at three months will document handicap after the initial period of recovery, while a one year assessment will capture any changes in handicap in the longer term. Any further changes after one year are likely to be small. making it unlikely that the additional information would offset the costs of a longer follow-up.

For the purposes of this thesis, this is a descriptive study examining the factors associated with handicap at two points in time. Although the data were collected in a prospective cohort format, the analyses are cross-sectional at each of the two follow-up periods. This approach generates a snap-shot description of handicap and its associated characteristics at the two clinically relevant periods of recovery. Such an approach was taken to complement the current body of research in this area which is descriptive in nature. While future research may involve more complex longitudinal analyses, the current focus of research in this area is aimed at trying to better understand the relationships between impairment, disability and handicap with particular emphasis on the role of environmental factors. Only when the dynamics of these static relationships are better understood will more complex analyses assessing change in handicap and its determinants over time become relevant. However, unlike typical cross-sectional designs, the prospective nature of this study permits an awareness of those subjects who died or who were lost to follow-up at the two assessment periods. Knowledge of these losses prevents inaccurate conclusions about the generalizability of the results, a problem which typically plagues crosssectional designs.

\section{Study Location}

This study was conducted at Sunnybrook Health Science Centre, a 1300 bed University of Toronto teaching hospital located in the City of North York within Metropolitan Toronto. Although this is a tertiary care facility, it acts as a community based centre with respect to stroke, receiving most of the stroke events in the surrounding residential area. Almost all stroke occurrences in Metropolitan Toronto are expected to be admitted to hospital at some point soon after onset, in contrast to cities in other countries, like Britain or New Zealand, where many episodes are treated at home (Christie, 1981; Cochrane, 1970). Therefore, by recruiting subjects from consecutive hospital admissions, it is likely that all stroke cases from the surrounding 
community would be included in this group. There may only be a small number who, because their symptoms are so mild, are seen in the office of their family physician or perhaps never receive medical attention at all. And one could argue that the impact of such a stroke on the lives of these people is so minimal, that it is, in effect, non-consequential with respect to handicap.

Approximately 200 stroke patients are admitted to Sunnybrook Hospital each year. $90 \%$ of whom come from the surrounding residential catchment area. The catchment area of over 352,000 individuals represents approximately $16 \%$ of the total Metropolitan population. and is composed of a predominantly elderly population of Anglo Saxon origin with higher education and income than the Metro Toronto population as a whole (Sunnybrook Health Science Centre. 1992).

An annual stroke register maintained at the hospital reveals that roughly $12 \%$ of stroke patients are diagnosed on admission as cerebral hemorrhage, and $88 \%$ as cerebral infarction, which is similar to proportions reported in population studies (Gordon, 1993). Each stroke patient is typically assessed by a multidisciplinary team (neurology, social work, physiotherapy, occupational therapy, speech therapy) who arrange pre-discharge consults and out-patient follow-up prior to acute care discharge.

\section{Subject Selection/Inclusion Criteria}

Consecutive strokes admitted to Sunnybrook Health Science Centre between August 1, 1990 and May 31, 1993 were eligible to participate in the study if they met the following inclusion criteria:

Stroke as defined by the World Health Organization (WHO MONICA Project, 1988) (but excluding subarachnoid hemorrhage and vertebrobasilar strokes) and classified as cerebral hemorrhage or infarct according to established clinical criteria (Foulkes, Wolf, Price, Mohr, \& Hier, 1988) (which uses information gained from computed tomographic scanning (CT) and clinical examination). Computed tomographic (CT) scanning was used to confirm the diagnosis of stroke in all subjects. 
The WHO and NINCDS criteria were used to standardize the definition and diagnosis of stroke in order to make the findings comparable to those from other studies'. However. subarachnoid hemorrhage was excluded because, although it is included in the WHO definition. it is managed differently from stroke, and typically falls under surgical rather than neurological care. For this reason, subarachnoid hemorrhage is not typically included in most medical studies of stroke (Johansson et al., 1992). Furthermore, the outcome of subarachnoid hemorrhage is different from that of other strokes, often resulting in sudden death before individuals reach medical care, which further limits their inclusion in clinical studies even if they were to fall under neurological management.

Strokes occurring in the vertebrobasilar territory were not included in this study because the primary research purpose was to assess the clinical determinants of recovery with the added value of cognitive deficits as predictors of outcome, and there was an a priori hypothesis that cognition is not affected by brain stem strokes. Damage to the vertebrobasilar system typically results in facial weakness, vertigo, nausea, vomiting, and loss of hearing, but because this part of the brain is not responsible for higher-order functions, cognitive deficits are not observed. By restricting the study to hemispheric events, the investigators were equalizing the chance of neurocognitive deficits among study subjects apart from the clinical characteristics of interest. Furthermore, most drug trials in stroke have focused almost exclusively on hemispheric strokes, and the investigators wanted to make the results of this study comparable to these trials. Vertebrobasilar strokes constitute approximately $15 \%$ of the total proportion of strokes (Chambers, Norris, Shurvel, \& Hachinski, 1987) and while they have a higher acute mortality rate, survivors tend to have a better long term survival than those with hemispheric infarcts or hemorrhages.

\footnotetext{
'Note that this definition does not include transient ischemic attacks (TIAs) which are episodes of temporary neurological dysfunction lasting less than 24 hours.
} 


\section{Instruments Used in the Collection of Information}

Like the ICD, the manual of the ICIDH is equipped with its own extensive classification system categorizing each component of the model using a series of codes (Badley, 1993; World Health Organization, 1980). For instance, impairments are classified and hierarchically rated under headings such as intellectual, other psychological, language, aural, ocular, visceral, skeletal, disfiguring or generalized. The disability classification similariy covers a broad range of human activity including behaviour, communication, personal care and locomotion. and each activity is further rated according to the amount of assistance required for performance (unaided, with assistance, or completely unable to perform). Finally, the handicap classification covers the six key dimensions of man's existence as a social being, as outlined earlier.

While this classification system exists, it is primarily a taxonomical tool and not ideally suited to measurement. Therefore, this research project used a number of different established measures to assess each component of the ICIDH model. This was done in order to maintain comparability with other studies in stroke outcome research which tend to use standardized measures rather than ICIDH codes. Additionally, the ICIDH manual is undergoing an extensive revision process, and it was considered preferable to use established measures rather than a classification system that could possibly become outdated. However, the measures that were used in this study closely reflect each component of the model, and incorporate much of the classification criteria used in the original coding manual. Each measure is outlined in tum below. There was no specific pre-testing of the instruments in this hospital setting, as they had all been previously tested for reliability and validity in other hospital populations of stroke patients, and it was assumed that any differences in this cohort would have minimal impact on the reported reliability and validity of the measures.

\section{i) Dependent Variable}

Handicap: The outcome variable of interest is handicap, defined by the WHO (World Health Organization, 1980) as "a disadvantage for a given individual that limits or prevents the fulfilment of a role that is normal (depending on age, sex, social and cultural factors) for that individual". The emphasis on social roles in this definition points to the social consequences of 
an illness, necessitating a tool which measures the activities of an individual as a social being. As outlined earlier, handicap can be a very personalized concept depending on the value an individual attaches to a particular activity, and is often influenced by cultural norms. Therefore, the assessment of handicap requires a subjective judgement of the disadvantages experienced by individuals in their particular social environment. Measures of handicap, however, are scarce (Hebert, Carrier, \& Bilodeau, 1988; Whiteneck, Charlifue, Gerhart, Overholser, \& Richardson, 1992; Harwood, Gompertz, \& Ebrahim, 1994), and where they do exist, they tend to be complicated, unreliable and have poor validity (Kimura, Chino, Saitoh, Sonoda, \& Domen, 1994). There is, therefore, a pressing need to develop a specific measure of handicap and validate it across diagnostic groups.

One approach is to use the Reintegration to Normal Living Index (RNL) (Wood-Dauphinee. Opzoomer, Williams, Marchand, \& Spitzer, 1988) to measure handicap. Reintegration to normal living is defined as "the reorganization of physical, psychological and social characteristics so that the individual can resume well adjusted living after incapacitating illness or trauma" (Wood-Dauphinee, Opzoomer, Williams, Marchand, \& Spitzer, 1988). This construct closely parallels the concept of handicap, and the index has been shown to be a proxy measure for quality of life (Wood-Dauphinee \& Williams, 1987) of which handicap is a part. The items in the measure were identified through a panel of patients, lay persons and health professionals as important to individuals in their return to usual living patterns after illness or trauma. These items are mobility, self care, daily and recreational activities, family and personal relationships. There was no identified hierarchy to these domains, and it was emphasized that the presence of symptoms or disability would not necessarily preclude reintegration into desired activities. Assistance from others or mechanical devices could allow individuals to participate in usual living patterns in spite of persisting disability. This is a notion which parallels the conceptual underpinnings of handicap. Because the RNL measures the extent to which individuals resume their usual living pattems after illness, a measure of reintegration to normal living measures the extent to which handicap is reduced following stroke.

The RNL is a self-report scale in which individuals are asked to rate their satisfaction with various aspects of their physical, emotional and social life, such as mobility in the home and 
community, participation in work, recreational and social activities, comfort with family roles and personal relationships (Appendix 2). These items closely mirror the six key dimensions of handicap outlined by the ICIDH which relate to the activities of individuals as social beings, and because the measure is concerned with the individual's satisfaction with these activities rather than their actual performance, the RNL focuses on handicap rather than disability. The measure asks respondents to answer eleven questions, each on an ordinal ten point scale, and the total score is proportionally converted to a score out of 100 by subtracting the minimum possible score of 11 from each total, then dividing by the range (99) and multiplying by 100 . This conversion transforms scores into more meaningful values ranging from 0 to 100 without changing the variance or the inherent relationships between the scores. The maximum possible score of 100 indicates complete reintegration to usual living patterns, or in other words. minimal handicap, while the minimum score of 0 indicates substantial handicap, with little return to usual social activities. Although the RNL is an ordinal measure, additive hierarchical scales such as this have been shown to be valid to use as a continuous variable for parametric analyses (Gaito, 1980). Therefore, the outcome measure in this study is analysed as a continuous variable.

One of the advantages of using the RNL in this study population is that it was developed in consultation with stroke patients and has been subsequently tested in studies of stroke survivors (Korner-Bitensky, 1993; Richards et al., 1993). The measure has demonstrated high internal consistency $(\alpha=0.90-0.95)$ and moderate interrater reliability when comparing scores from respondents and significant others (Wood-Dauphinee, Opzoomer, Williams, Marchand, \& Spitzer, 1988). Construct validity was demonstrated when compared to indices of quality of life (Spitzer, Dobson, Hall, \& Chesterman, 1981) and psychological well-being (Bradburn, 1969) and content validity was also assured during the development of the index (Wood-Dauphinee, Opzoomer, Williams, Marchand, \& Spitzer, 1988) through consultations with individuals who had been successful in returning to normal lifestyles after disablement. The RNL has also been shown to be sensitive to change in clinical status (Wood-Dauphinee, Opzoomer, Williams, Marchand, \& Spitzer, 1988). The established reliability and validity of this measure, along with its strong parallelism with the concept of handicap, makes it the most suitable measure to assess 
the outcome variable of interest in this study. The RNL was used in this study to measure the level of handicap at the three month and one year follow-up periods after stroke.

\section{ii) Independent Variables}

Impairment: In contrast to the more socially oriented concept of handicap, impairment represents the physiological impact of disease at the level of the body or organ. A standardized hemispheric stroke scale (Adams, Meador, Sethi, Grotta, \& Thomson, 1987) was used to assess the magnitude of an individual's physical and neurocognitive impairments due to stroke. This scale assesses impairments in consciousness, vision, language, and motor and sensory functions following acute stroke, highlighting the physical and neurocognitive consequences of stroke as outlined in the impairment classification of the ICIDH (World Health Organization, 1980). It also has the advantage of being able to detect aphasia and neglect (typically not captured by other stroke impairment scales (Cote, Hachinski, Shurvel, Norris, \& Wolfson, 1986)) which have the potential to affect functional independence and handicap. This impairment scale was designed to be repeatedly applied by a neurologist in less than 30 minutes, and sensitive enough to detect changes in physical impairments and higher cortical function. The measure uses an ordinal scale to assess subjects in five dimensions of impairment, with higher scores indicating greater impairment (Appendix 3): level of consciousness (on a 12 point scale), language (20 points), other cortical function (including aphasia and neglect) (17 points), inotor function (40 points), and sensory function (11 points). A maximum score of 100 is therefore possible if an individual is severely impaired in all domains. A minimum score of zero, indicates no impairment following stroke.

Because this measure uses an ordinal scale with 100 points divided into five components, analyses using the raw scores would be complicated and confusing. Therefore, a categorical impairment variable was derived by allocating the final score for each subject into one of three impairment categories (mild, moderate and severe) as outlined by the authors in the original reference (Adams, Meador, Sethi, Grotta, \& Thomson, 1987). Specifically, a final raw score between the values of 0 and 20 inclusive was labelled as a "mild" impairment, 21 to 50 as "moderate", and scores over 50 were labelled as "severe". These global neurological categories 
were found to be highly correlated with these particular scores when testing the validity of the measure ( $r=0.89$ ) (Adams, Meador, Sethi, Grotta, \& Thomson, 1987).

The Adams' Hemispheric Stroke Scale has been found to be a reliable measure when assessed for interobserver reliability $(r=0.95)$ (Adams, Meador, Sethi, Grotta, \& Thomson. 1987). Additionally, during the first year of the study, two other stroke impairment scales, the NIH scale (Brott et al., 1989) and the Canadian Neurological Scale (Cote, Hachinski, Shurvel, Norris, \& Wolfson, 1986), were used in addition to the Adams' Scale in order to make a comparative assessment of its performance. After comparing scores obtained in the first year of data collection, the investigators found the Adams' Scale to be the most sensitive and comprehensive of the three measures, particularly with mild impairment since it sampled the domains of impairment, particularly language, more completely. The other two measures were subsequently dropped from use in the study.

However, while the Adams' scale documents physical and neurocognitive impairment after stroke, it fails to detect psychological impairment, such as depression, which falls under the category of emotion, affect and mood in the ICIDH impairment classification. Depression is a common sequela after stroke, estimated to affect between $30 \%$ and $60 \%$ of survivors (Eastwood, Rifat, Nobbs, \& Ruderman, 1989; Robinson \& Starkstein, 1990) and often remaining with individuals up to two years post stroke (Parikh et al., 1990; Robinson, Starr, Kubos, \& Price, 1983).

While it was originally thought that depression was an emotional reaction to the devastation of stroke, it was subsequently suspected to have an anatomical or physiological basis since depression was found to occur more often in people with lesions in the frontal lobe of the left hemisphere of the brain (Robinson, Kubos, Starr, Rao, \& Price, 1984). Several studies have found that stroke patients with lesions in the left frontal lobe have a significantly higher frequency of depression than those with more posterior lesions in the left hemisphere or those with right hemisphere lesions (Starkstein \& Robinson, 1993). Furthermore, empirical studies have only demonstrated a weak correlation between the severity of impairment and the severity of depression (Starkstein \& Robinson, 1989) suggesting that the depression is not simply an emotional response to the severity of the stroke. The fact that post-stroke depression responds 
well to pharmacologic therapy in randomized controlled trials (Lipsey, Robinson. \& Pearison, 1984; Reding, Orto, \& Winter, 1986) coupled with the suggestive evidence of a physiological difference between depressed and non-depressed individuals, lends support to the classification of depression as an impairment rather than a psychological outcome following stroke.

Post-stroke depression has been shown to adversely affect mobility (Mayo, Komer-Bitensky, \& Becker, 1991), social activities (Feibel \& Springer, 1982), coping ability (Sinyor et al., 1986). and cognitive impairment (Robinson, Bolla-Wilson, Kaplan. Lipsey, \& Price, 1986) in stroke survivors. It therefore has the potential to affect life habits, or handicap, after stroke. and should be included in the analysis as an important source of stroke-induced psychological impairment.

Because depression is not picked up with the hemispheric stroke scale, it was measured separately using the Zung Self-Rating Depression Scale (ZDS) (Zung, 1965) (Appendix 4) at the three and twelve month follow-up periods. The ZDS is a 20 item self-report impairment index in which individuals respond to questions about somatic symptoms most commonly used to characterize depressive disorders (insomnia, loss of appetite, constipation, fatigue and sexual disinterest). Respondents indicate the frequency of symptoms on an ordinal four point scale ( 1 $=\mathrm{a}$ little of the time, $2=$ some of the time, $3=$ a good part of the time, $4=$ most of the time). A final score is derived by dividing the sum of the values by the maximum possible score of 80 in order to obtain a decimal value. The score is then categorized as a nominal dichotomous variable using a cutpoint score of 0.50 or higher to designate the presence of depressive symptoms (Zung, Broadhead, \& Roth, 1993; Zung, 1990).

When comparing the Zung scale to standards based on the Diagnostic and Statistical Manual for Mental Disorders (DSM-III-R) (American Psychiatric Association, 1987), the measure demonstrated a sensitivity of $97 \%$, specificity of $63 \%$, positive predictive value of $77 \%$ and negative predictive value of $95 \%$ (Zung, Magruder-Habib, Valez, \& Alling, 1990). The reliability and validity of this measure in brain-injured populations have also been demonstrated (Robinson, Kubos, Starr, Rao, \& Price, 1984; Robinson, Starr, Kubos, \& Price, 1983).

Disability: Disability represents the impact of disease at the level of the person's day to day activities. This includes self care and mobility, often referred to as activities of daily living or instrumental activities of daily living. Since functional independence is a major goal of 
rehabilitation therapy, measures of disability are often developed in rehabilitation settings. The Functional Independence Measure (FIM) (Keith, Granger, Hamilton \& Sherwin, 1987) is one such measure that was developed by a national task force sponsored by the American Academy of Physical Medicine and Rehabilitation and the American Congress of Rehabilitation Medicine in recognition of the need for a more sensitive tool in rehabilitation medicine to detect clinically important change in a uniform and reliable manner (Granger \& Hamilton, 1990). The FIM is a basic measure of disability, designed to assess what an individual actually does, not what he or she ought or might be able to do. Because disability encompasses the performance of activities in a relatively neutral way, it does not include the meaning of the activity in the persons's life as covered by handicap. The FIM is widely used in rehabilitation centres and is becoming a popular instrument in stroke outcome research (Bohannon, Kloter, \& Cooper, 1991; Wilson, Howe, \& Keith, 1991; Oczkowski \& Barreca, 1993). The advantage of this measure over other disability measures, such as the more established Barthel Index (Granger, Hamilton, Gresham, \& Kramer, 1989), is that not only is it more sensitive, but it also includes an assessment of communication and cognition, two particularly important components of social and functional activity after stroke (Anderson, Bourestom, Greenberg, \& Hildyard, 1974; Mayo, Korner-Bitensky, \& Becker, 1991; Woo, Kay, Yuen, \& Nicholls, 1992), which are not typically captured in other disability indices. Because of these advantages, the FIM was chosen as the disability measure in this study and was used to assess subjects at all follow-up periods.

The FIM is an 18 item, seven level ordinal scale that is used to assess ability in four domains of daily functioning (see Appendix 5) that encompass many of the categories of daily life activities included in the disability classification of the ICIDH. There are eight items on self care (eating, grooming, bathing, dressing upper body, dressing lower body, toileting, bladder and bowel management), five items on mobility and locomotion (transfers to bed/chair, toilet and tub/shower transfer, walking/wheelchair and stair climbing), two items on communication (comprehension and expression) and three items on social cognition (social interaction, problem solving and memory). Individuals are assessed on each item using standardized scaling descriptors described in the FIM Guide (Data Management Service of the Uniform Data System for Medical Rehabilitation, 1990) and scored on an ordinal 7 point scale. A score of 1 or 2 
indicates that an individual is completely dependent on others for the performance of an activity, 3-5 indicates that they require varying degrees of assistance from others, and a score of 6 or 7 indicates that an individual can function independently. The maximum possible score is 126. representing complete independence in all 18 items of daily function, whereas a score of 18 represents complete dependence.

The nurse coordinator in this study was formally trained in the use and scoring of the FIM at a training and testing workshop hosted by the developers of the scale, where different scenarios were used to illustrate the techniques of the scoring system. A set of standardized questions was used to determine the ability level of each subject without actually observing each activity. The FIM takes approximately 20 minutes to administer.

One of the advantages of using the FIM is that it has been extensively tested for reliability and validity in rehabilitation patients, often in stroke survivors. The measure has demonstrated good interrater reliability, both for the overall score and separate subscores (Hamilton, Laughlin, Granger, \& Kayton, 1991) using intraclass correlation and Kappa coefficients, and has been shown to be internally consistent (Dodds, Martin, Stolov, \& Deyo, 1993). The FIM has also demonstrated good validity as a measure of disability in rehabilitation settings. Face and content validity were documented using a Delphi panel of rehabilitation experts (Granger. Hamilton, Keith, Zielezny \& Sherwin, 1986). Construct validity was found to be adequate when measuring its association with several clinical and demographic indicators of functional impairment and disease severity (Dodds, Martin, Stolov, \& Deyo, 1993) and when comparing it to scores from similar scales in stroke survivors (Granger, Cotter, Hamilton, \& Fiedler, 1993). The FIM has also proven to be sensitive in detecting improvements in disability over time (Dodds, Martin, Stolov, \& Deyo, 1993; Hamilton \& Granger, 1994). The solid reliability and validity of this measure makes it ideal for use in this study.

Although the FIM is scored on an ordinal scale, the overall FIM score has been validated as a continuous measure (Linacre, Heinemann, Wright, Granger \& Hamilton, 1991; Heinemann, Linacre, Wright, Hamilton, \& Granger, 1993) using the statistical technique of Rasch analysis (Rasch, 1980) which constructs interval measures from ordinal data. This technique revealed that the FIM could be validly scaled as two interval measures, one motor component combining 
the 13 items on self-care, mobility and locomotion, and one cognitive component combining the 5 items on communication and social cognition (Linacre, Heinemann. Wright, Granger \& Hamilton, 1991; Heinemann, Linacre, Wright, Hamilton, \& Granger, 1993). However, for the purposes of this thesis project, these two interval measures were then categorized into two nominal variables because discrete categories tend to be more intuitively meaningful than interval values when considering levels of ability. Two categorical variables were created, each with three aggregate levels, as follows.

The raw FIM scores for the combined 13 motor items and the combined five cognitive items were converted into two trichotomous categorical variables, with the categories independent, moderately dependent and dependent in either motor ability or cognitive ability. The cutpoints chosen to delineate these categories follow the methods of Granger and colleagues (Granger. Hamilton, Linacre, Heinemann, \& Wright, 1993) who found that when plotting the raw FIM scores for both the motor and cognitive components against the Rasch converted scores, the slopes changed at two points suggesting the presence of three different subgroups along the scale. Granger et al. used these points to compare high and low levels of functional ability, and similarly, these cutpoints were used to delineate three levels of ability in this study. For the motor component, these cutpoints were the raw motor FIM scores of 29 and 71 and these points were used to categorize motor ability into dependence (raw motor FIM scores 13 to 29 inclusive), moderate dependence (raw scores of 30 to 71 inclusive) and independence (raw scores of 72 to 91 inclusive). For the cognitive component, the raw cognitive FIM scores of 12 and 27 were identified as indicative of differences between dependence (raw cognitive FIM scores of 5 to 12 inclusive), moderate dependence (raw scores of 13 to 27 inclusive) and independence (raw scores of 28 to 35 inclusive).

Environmental Factors: Environmental factors can be physical or social, creating or preventing obstacles that limit an individual's participation in usual life activities. A number of variables were collected in this study to account for the importance of environmental factors as outlined earlier.

First, in terms of the social resources available to individuals, family organization and structure were documented by collecting nominal information on each subject's marital status 
(single, married, widowed, divorced, separated) and living situation (alone, with family, with friends) at stroke onset, and throughout the one year follow-up period. Post-stroke handicap has been shown to be minimized when there is a spouse caring for the patient at home (Evans, Bishop, \& Haselkorn, 1991), and family organization has also been shown to be an important predictor of functional outcome after stroke (Ferruci et al., 1993; Silliman, Wagner, \& Fletcher. 1987; Wade \& Hewer, 1987). Information was also collected on place of residence throughout the follow-up period (home or institution).

Other resources, such as health services, are also important factors that could potentially prevent handicap situations ${ }^{2}$. The use of rehabilitation services is particularly important in the stroke population because such services have been shown to influence successful recovery (Smith, Goldenberg, \& Ashburn, 1981; Stevens, Ambler, \& Warren, 1984). For instance, a randomized controlled trial suggested that stroke survivors who received an intensive course of rehabilitation (four whole days per week) reported significantly more improvement in functional ability than those who did not receive any rehabilitation (Smith, Goldenberg, \& Ashburn, 1981). Therefore, rehabilitation therapy may help persons to adapt to or cope with their residual disabilities so that handicap is minimized. The use of rehabilitation services was documented for all study subjects (yes/no) either on an outpatient basis or admission to a rehabilitation facility prior to the 3 month or one year follow-up periods.

Finally, wealth falls under the general category of environmental resources, and socioeconomic status (SES) has repeatedly been shown to be an important factor influencing health and life quality (Estes \& Rundall, 1992; Longino, Warheit \& Green, 1989; Hirdes \& Forbes, 1992). Individuals with higher SES may be more able to obtain assistive devices and services that allow them to minimize handicap than those with lower SES. Level of education was the only variable collected in this study that approximates SES, and was obtained at hospital admission using a nominal scale (no education, primary school, high school, college, university).

\footnotetext{
2 The fact that all subjects in this study received neurological assessments and follow-up by a study team three times over a one-year period as part of the study protocol, can be considered a health and social service resource in itself. But the fact that all subjects received this service unites them under this common factor, negating its inclusion as a separate variable. However, it should be noted that not all stroke victims would be able to experience the benefits of these services and any generalizations should be made with this fact in mind.
} 
Information on other environmental factors as outlined by the ICIDH (Canadian Society for the ICIDH, 1991) such as political and governmental structures, architecture, technology, climate and cultural values and attitudes were not collected in this study due to limited feasibility and an already large amount of accrued data, yet their underlying importance in the handicaps creation process cannot be ignored and will be explored in the discussion section.

\section{iii) Other Predictor Variables}

Information was also collected on other important variables outside the ICIDH system because they were known or were projected to influence post-stroke handicap. It was postulated that these variables could either affect handicap independently, create spurious relationships (confounding), or alter the relationships between the dependent and independent variables at different levels (effect modification). Confounders are those factors related to both the predictor and outcome variables of interest which "wholly or partially account for the apparent effect of the study exposure, or that mask an underlying true association" (Schlesselman, 1982). Effect modification occurs when the relationship between the predictor and outcome variables of interest varies according to the level of a third variable. The following variables may either have independent, confounding or interactive relationships with stroke handicap, and their effects will be explored in the data analysis and discussed in the results section of this thesis.

In the first place, age of each subject was recorded at onset, and was collected because of its potential relationship to both the predictor and outcome variables. According to data provided by the Framingham study, stroke survivors over the age of 65 have twice the disability and four times the activity limitations as those between the ages of 45-64 years (Gresham, Philips, \& Wolf, 1979). Older individuals are more likely to have more severe impairments and disabilities following stroke (Ferruci et al., 1993; Gowland, 1982), and increasing age may be associated with greater handicap (Grimby et al., 1993) and compromised life quality (de Haan, Limburg, Van der Meulen, Jacobs, \& Aaronson, 1995), apart from the presence of overt disabilities or impairments, since older persons may experience a greater restriction in their social roles. Consequently, age is a potential confounder since it could appear that impairments and disabilities are positively related to handicap when, in fact, age is the underlying reason, being 
a common factor to both. Therefore, age needs to be taken into account in the analysis in order to determine its independent effect on handicap and also the impact of stroke severity and disability on handicap across people of the same age.

Although age was collected as a continuous variable, it was subsequently collapsed into a three level categorical variable based on the different clinical characteristics expected of young ( $<50$ years of age) middle aged (50-75 years of age) and older ( $>75$ years) strokes. Only 10-15\% of strokes occur before the age of 50 (Millikan, McDowell \& Easton, 1987a) and these events are most likely cardiogenic since atherosclerosis is not sufficiently developed by this age to be the source of the event. Recovery may also be more swift in this group since there may be fewer underlying comorbid conditions. The majority of strokes occur in individuals between the ages of 50 to 75 years, contributing most to the epidemiological stroke profile. While only a minority of all strokes occur in those individuals who are over the age of 75 , recovery is likely more difficult since there are a greater number of underlying conditions and overall frailty.

Pre-stroke level of disability is also a potential confounder. Persons with more disability before their stroke may be more likely to report more disabilities after their stroke regardless of the impact of the stroke (Colantonio, Kasl, \& Ostfeld, 1994), and may also be more likely to report greater handicap since pre-morbid disability is likely to restrict life activities. This situation could make it appear that post-stroke disabilities create handicap situations, when in fact it is due to the underlying presence of pre-stroke disability. Therefore, pre-stroke ability was documented retrospectively at hospital admission using the FIM (Keith, Granger, Hamilton \& Sherwin, 1987) in consultation with the subject, hospital chart and close family, when available ${ }^{3}$.

\footnotetext{
${ }^{3}$ However, it should be noted that a retrospective assessment of ability at the point of hospital admission may potentially affect the accuracy of reporting. For instance, an individual with a mild stroke may retrospectively report a good level of ability whereas a person with a more severe stroke may be so devastated that all life looks bleak, including pre-stroke disability. Or, conversely, more severe stroke survivors may report a more optimistic pre-stroke disability while those with only minor strokes may be more realistic about disability level before the stroke. Therefore, a retrospective assessment of pre-stroke disability at the time of admission may be subject to misclassification. A possible solution to these problems would be to validate this pre-stroke level of disability through family physician's records, for example, but unfortunately, this was not feasible in this study due to time and resource constraints. But, although it was not possible to validate this data, the potential effects must nevertheless be considered in the interpretation of results.
} 
Comorbid conditions or a history of other illnesses could also influence the level of handicap in stroke survivors, apart from the current stroke-induced impairments and disabilities. Individuals with other diseases (Parkinsonism, osteoarthritis, heart failure) have been found to have a worse functional outcome after stroke (Kelly-Hayes, 1990) because of increased complications in recovery. A history of previous stroke has also been shown to elevate the risk for poor outcome (Aho, Harmsen, Hatano, \& et al, 1980; Bonita, Beaglehole, \& North, 1984) by increasing the resulting impairments and disabilities. Therefore, comorbid conditions could also act as confounders since they are associated with greater impairments and disabilities and could also act to increase handicap after stroke, apart from the current stroke related sequelae. So again, comorbid conditions could make it appear that impairment and disability are related to handicap, when in fact it is due to the common factor of comorbid conditions. Information on these variables was collected at hospital admission and dichotomized as a categorical variable (present/absent) in order to control for their effects in the analysis and to assess any independent effects on post-stroke handicap.

The presence of intervening illnesses occurring after stroke onset could also affect the level of handicap reported, but not as a confounder because these illnesses occur after the resulting stroke-induced impairments and disabilities. However, the sequelae from the stroke could elevate the risk for other illnesses and therefore increase handicap, making it important to separate the effects of other illnesses on post-stroke handicap from stroke-induced impairments and disabilities. Information on intercurrent illnesses was documented at all follow-up visits and dichotomized as a categorical variable (yes/no) and classified as stroke related or non-stroke related.

It was also important to document an individual's level of handicap prior to their stroke. If individuals were restricted in their usual social roles before their stroke, then regardless of their stroke-induced impairments and disabilities, they may continue to experience greater handicap throughout the follow-up period. Again, this variable is not a confounder because although it may be associated with post-stroke handicap, it is not related to impairments and disabilities. Therefore, its effects will be assessed as an independent factor. However, it was not possible to use the handicap instrument ( $R N L)$ retrospectively because the measure is only designed to 
assess handicap at the point of administration. Therefore, a different measure, the Spitzer Quality of Life Index (Spitzer, Dobson, Hall, \& Chesterman, 1981) (Appendix 6) was administered to each patient on admission since it asks respondents questions pertaining to their life in the preceding week.

This self-report index was designed to measure general well-being in individuals with chronic disease using five themes identified through pilot testing as important to quality of life: activity level (including occupation), activities of daily living, health, quality of social support, and psychological outlook. Respondents assign a score of 0,1 or 2 to each of these five themes, indicating increasing well-being, and summing to a maximum possible score of 10 . Healthy individuals generally score an average of 8 or 9 on the scale (Spitzer, Dobson. Hall, \& Chesterman, 1981), while those living with chronic disease have a mean score between 7.1 and 7.3. Seriously ill individuals have been shown to have much lower scores, with a mean of score 3.3 (Spitzer, Dobson, Hall, \& Chesterman, 1981). The measure has been found to be internally consistent and reliable when administered by different physicians within seven days. It has also been judged to be valid, when comparing it to a more extensive index of quality of life, and when assessed by a panel of patients, healthy individuals, physicians and research methodologist (Spitzer, Dobson, Hall, \& Chesterman, 1981).

Although the Spitzer Index is not specifically a handicap measure, it nevertheless captures some of the life habits identified as being key dimensions of handicap (Badley, 1993) such as recreational and work activities, mobility, self care and social relationships that may help to reveal the level of handicap experienced by subjects prior to their stroke. Also, by being an overall measure of quality of life, it may capture a broader life picture which could have an impact on handicap, over and above the stroke experience.

Sex of the subject may also be a factor independently affecting handicap since men and women may cope with the impairments and disabilities following stroke differently. For instance, evidence suggests that women may be more effective in mobilizing supportive resources than men (McMullen \& Gross, 1983), which may affect the extent to which impairments and disabilities impact on handicap. Gender could, therefore, act as an effect 
modifier if the effects of stroke on handicap varied according to the sex of the subject. Sex was analyzed as a dichotomous categorical variable (male/female).

Finally, from a clinical standpoint, side and type of stroke are also important variables to consider when looking at the determinants of handicap. Individuals with right hemispheric strokes have been shown to have an increased likelihood of dependency in activities of daily living (Johansson et al., 1992) following their stroke. Individuals with a right hemisphere stroke are also more likely to experience spatial and perceptual deficits, which tend to be more difficult to detect. This, coupled with the fact that these individuals also tend to overestimate their abilities, means that they may not receive as much therapy and counselling for their disabilities as those with left hemispheric lesions, and therefore, may have an elevated risk for handicap. Quality of life has also been found to be compromised in individuals with right hemispheric lesions compared to those with left sided lesions (de Haan, Limburg, Van der Meulen, Jacobs, \& Aaronson, 1995). Individuals with cerebral hemorrhages have been shown to be at elevated risk for early mortality compared to those with cerebral infarcts (Kelly-Hayes, 1990; Sacco, Wolf, Kannel, \& McNamara, 1982; Chambers, Norris, Shurvel, \& Hachinski, 1987), although there has been no evidence of a difference in handicap in those who survive the acute stage (de Haan, Limburg, Van der Meulen, Jacobs, \& Aaronson, 1995). Therefore, although stroke type is not a predictor of handicap in itself, it is an important fact to document in order to ascertain which type of stroke is more likely to survive to a point where handicap becomes meaningful. Therefore, side (right brain side, left brain side or bilateral lesions) and type (cerebral hemorrhage or infarct) of stroke, as documented by clinical examination and CT scan, were included in the analysis.

\section{Procedures Used in the Collection of Information}

This was a prospective cohort study of four years duration in which subjects were recruited over a three year period, with one additional year for follow-up. All stroke patients admitted to Sunnybrook Health Science Centre who met the inclusion criteria were approached by the nurse coordinator who explained the purposes and procedures of the study. Informed consent was then obtained from all eligible subjects agreeing to participate (see consent form Appendix 7). For 
some patients who were too ill or confused at onset it was occasionally possible to obtain a substitute informed consent from a family member'. The nurse coordinator then obtained information on demographics, medical history as well as a retrospective assessment of pre-stroke ability (using the FIM) and quality of life (using the Spitzer Quality of Life Index). Assistance from caregivers and the patient's hospital chart were used to obtain more information where possible. The study neurologist (S.E. Black) or the neurology fellow examined each subject within three days of admission and scored them using the stroke impairment measure.

All consenting subjects returned to the hospital outpatient clinic for follow-up at three and twelve months after stroke onset. At these visits, the neurological assessment was repeated by the neurologist, and the nurse coordinator administered the disability and handicap measures. The total time for each follow-up visit was approximately three hours, which included all clinical assessments required for the larger study. The specific follow-up for the handicap component of the study lasted anywhere from 40 to 90 minutes, depending on the patient.

Information on each study subject was collected in a file, and all data were subsequently coded and entered into a Macintosh computer. One data entry clerk assisted the nurse coordinator with this task. The data were originally entered into a database using Microsoft Excel (Microsoft Corporation, 1994), but later changed to FileMaker Pro (Claris Corporation, 1993). All written information was stored in a locked filing cabinet, and the computerized database was kept in a locked room of the research laboratory. The final data set was subsequently converted into a SAS file so that statistical analyses could be performed using the Statistical Analysis System (SAS) (SAS Institute Inc., 1985a; SAS Institute Inc., 1985b). For reasons of confidentiality, this data set included only study numbers, omitting patients' names.

\section{Statistical Analysis Strategies}

The objective of this thesis is to examine the extent to which impairments and disabilities following stroke affect handicap in interaction with environmental factors. The three month and

\footnotetext{
${ }^{4}$ Substitute informed consent is often used in studies of stroke patients where the initial sequelae of stroke prohibits informed consent from the patient. Substitute consent from a caregiver allows individuals to enter studies at onset, giving them the opportunity to be a participant throughout the follow-up period if they are able.
} 
one year follow-up periods were chosen to illustrate the psycho-sociological impact of stroke in both the subacute and more chronic stages of stroke recovery. Exploratory data analysis was first undertaken to generate summary descriptive statistics on the independent and dependent variables of interest as well as on potential confounders and other variables hypothesized to be important. All analyses were performed using SAS (SAS Institute Inc., 1985a; SAS Institute Inc., 1985b). Measures of central tendency and spread were generated for the continuous variables, while categorical variables were summarized with frequency counts and bar graphs. These summary statistics were also used to verify the assumptions underlying the proposed statistical analyses. -

In order to describe the basic first level relationships between impairment, disability and handicap, simple bivariate analyses were first performed to examine RNL scores by the independent variables. For the categorical independent variables, one-way analysis of variance (using the SAS procedure GLM (SAS Institute Inc., 1985b) for unbalanced data sets) was used to compare RNL means between groups. Post-hoc multiple comparisons using Tukey's studentized range test (one of the more conservative tests) were used to examine the specific differences between means. For categorical variables with only two groups, t-tests were used. For the continuous independent variables, simple linear regression was used to examine the bivariate relationships with RNL. Statistical significance was assessed using a two-tailed ${ }^{5}$ alpha of 0.05 to conform with standard statistical practice.

Finally, multivariate analyses were performed to ascertain the independent predictive effects of impairment and disability on handicap after controlling for the effects of other factors, including potential confounders. Particular attention was paid to the importance of interaction effects between impairment, disability and environmental factors. These analyses were performed using generalized linear models. The main effects of the impairment and disability variables on handicap were first examined using multi-way analysis of variance. The other potentially important categorical variables were then successively introduced into the statistical

\footnotetext{
${ }^{5}$ Two-tailed hypothesis testing indicates no preconceived direction of the effect of impairment or disability on handicap. Although it is hypothesized that more impaired individuals will report greater handicap, this is not certain, and two-tailed hypothesis testing allows for the detection of an effect in both directions.
} 
model, with continuous variables introduced as covariates. Multiplicative interaction terms involving the environmental variables with impairment and disability were also added. Once a variable became significant in the multivariate analysis, it was kept in the model until another variable made it unimportant. Again, all decisions to keep variables in the model were made based on an alpha of 0.05 conforming with standard statistical practice. Models were compared based on their multiple squared correlation coefficients $\left(\mathbf{R}^{2}\right)$. Particular attention was paid to potential confounding effects and correlations between independent variables by examining any changes in parameter estimates and their standard errors obtained with the "solution" statement in the SAS procedure GLM (SAS Institute Inc., 1985b).

Because individuals in this study were re-entered into the study as new cases following a further stroke, individuals could potentially accumulate data on two or more events. Although one approach could use strokes as the unit of analysis, this would violate one of the fundamental assumptions underlying parametric statistical tests such as t-tests and analysis of variance: that of the independence of observations. Fourteen individuals in this study cohort had second strokes throughout the study follow-up period for which data was available on two events. However, of the 14 second strokes, ten of them occurred before the first three month follow-up period, preventing any repeated assessments throughout the study period. For the remaining four individuals, three were not followed twice either because they were too sick or because they had died at the time of the second follow-up. Only one individual was assessed twice at the three month follow-up period because the second event occurred after the first three month assessment (but before one year), and the person then went on to have an additional three month assessment following the second event and then a one year assessment. In order to prevent the violation of the assumption of independent observations, the data for the first three month assessment was deleted from the data set for this one subject, and all statistical analyses were performed using information from the most recent event only. The fact that a subject had had a previous stroke was statistically controlled for in the analysis. 


\section{Sample Size Requirements}

In order to detect a difference in mean handicap scores between the various impairment and disability groups (if indeed there is one), a sufficient number of observations is required in each subgroup of the independent variable. Because there are no specific sample size formulae for multivariate models, an estimate of the required sample size can only be approximated using a formula for univariate situations. For this analysis, sample size calculations were performed using the formula for analysis of variance (Norman \& Streiner, 1994), which is based on the designated alpha and beta significance levels and the hypothesized effect size.

Alpha is the probability level at which it is arbitrarily decided that it is unlikely that the difference between handicap scores is due to chance alone. The conventional alpha of 0.05 indicates that there is a $5 \%$ chance that the null hypothesis is rejected when in fact there is no difference between groups. For this study, a two-tailed alpha was set at 0.05 conforming with standard statistical practice because it was preferable to minimize the chance of a Type I error (claiming that there is a relationship when in fact there is none) at this point in the theoretical development of the ICIDH. Any false relationships would impede the theoretical development of the ICIDH model. Therefore, it was preferable to minimize the probability of a Type I error, even at the expense of an increased risk of a Type II error (failing to reject the null hypothesis when in fact there is a difference in handicap scores between the different impairment and disability groups). Therefore, a two-tailed beta was set at 0.20 allowing for a $20 \%$ probability of a Type II error, which was assumed to be a less serious consequence.

The effect size is the effect of the group differences in levels of impairment and disability on handicap scores. Because little research has been done in this area, there is no previously established difference in RNL means across impairment and disability groups to use as a basis for a postulated effect size. Therefore, for the purposes of these sample size calculations, an effect size can only be chosen arbitrarily. Since the RNL Index ranges along a scale from 0 to 100 , a difference of 10 points between the levels of impairment or disability would indicate an increase or decrease in handicap which, in the absence of any pre-established difference, can be considered a meaningful change in handicap from the individual's perspective. 
Given these assumptions, sample size calculations can then be performed. However, the specific sample size requirements for analysis of variance outlined by Cohen (Cohen, 1977) require further assumptions to be made about the dispersion of means within this effect size. Under these requirements it was further assumed that the different RNL means are distributed evenly within this ten point difference and that, as a conservative estimate, the standard deviation of this distribution is eight points. Therefore, in order to detect a statistically significant ten point difference in handicap scores amongst the different levels of impairment and disability with a conventional two-tailed alpha of 0.05 and a two-tailed beta of 0.20 , a minimum of 14 subjects is needed in each of the three impairment and disability groups Norman \& Streiner, 1994).

\section{Ethical Considerations}

In order to conduct this study, the project first had to be approved by the Research Ethics Board at Sunnybrook Health Science Centre. All research projects involving Sunnybrook patients must obtain ethics approval from this Board, which ensures that research involving human participants is scientifically and ethically sound. In considering each project, the Board assesses the scientific methodology of the study as well as the potential for risk, harm, discomfort and benefit to study subjects. All projects must include a consent form and separate clinical information form explaining the study to participants. This project, including consent forms (Appendix 7) was considered by the Research Ethics Board to be ethically acceptable, and was passed on July 12, 1990 (see letter of approval - Attachments).

When each subject was approached by the nurse coordinator following admission, the objective and procedures of the study were clearly explained and the patient was invited to participate in the study. Patients were informed that they were under no obligation to participate in the study and that their medical care would in no way be compromised if they chose not to participate. They were also informed that they were not necessarily going to benefit directly from being a study participant. The nurse coordinator then gave the patients time to think about participation, leaving them a copy of the information sheet for review, and encouraging them to 
discuss it with family members. The nurse coordinator made herself available to patients and their families to answer questions the may have had throughout this time.

All patients who subsequently agreed to participate then signed the consent form, which was kept on file in the subject's chart. At the time this study was conducted, it was legally and ethically acceptable to obtain a substitute informed consent from a close family member for patients who were too ill or confused to make an informed decision to participate in this study. A substitute informed consent was obtained from a small number of subjects in this study (see surrogate consent form - Appendix 7). Surrogate consents were provided either by the spouse or offspring of the patient, and the same procedures were followed for collecting consent as for directly consenting subjects.

All participating subjects were provided with the names and telephones numbers of the study neurologist and nurse coordinator in case they needed to reach them for questions or concerns. All subjects were informed that they were free to withdraw from the study at any time without any threat to their care. All data collected in the study were kept strictly confidential, with all data collection forms in a locked filing cabinet. The computerized data base was kept in a locked research laboratory. Information was only released to other members of the medical team involved in the patient's care on the agreement of the subject.

While the clinical component of the study involved repetition of an observational brain imaging technique that may not have been prescribed in routine patient care, the handicap component necessitated no procedure that would not otherwise have been provided in the care of a stroke patient. However, the handicap assessment required patients to respond to potentially personal and sensitive questions that may have been uncomfortable, and indeed, a small number

of consenting subjects subsequently refused to complete the handicap component because they found it to be too emotional or personally intrusive (see discussion in Reasons for NonParticipation). On the other hand, similar psychosocial research has been shown to be helpful to some patients because it allows them to express emotions and feelings that they would otherwise have little opportunity to do so (Eardley, Cribb, \& Pendleton, 1991). 


\section{RESULTS}

\section{DESCRIPTIVE STATISTICS}

\section{i) All Eligible Subjects}

Four hundred and fifty consecutive hemispheric strokes were admitted to Sunnybrook Health Science Centre between August 1990 and June 1993 and therefore eligible to be enrolled in this study. This constituted 436 individuals, 14 of whom went on to have a second stroke within the first year of follow-up. These descriptive statistics, therefore, refer to 436 individuals, although data that refer to stroke characteristics (stroke type and side), are reported for all 450 events.

The tables in Appendix 8 give the basic demographic profile for all patients which is routinely collected at the time of their admission to hospital, and it is evident that these individuals were not substantially different from those reported in other stroke populations. Roughly half of the subjects were male, which is similar to proportions in other community studies, although the age-standardized incidence rates are usually higher in males than females (Gordon, 1993) with the male/female ratio reported to be about 1.33 (Kurtzke, 1986). The more equal gender balance in this study may be a result of the higher age of the individuals in the hospital catchment area, which would likely include a higher proportion of women due to a greater overall life expectancy in females.

The average age of the subjects was 74.9 years $( \pm 11.6)$ which is indicative of the greater incidence of stroke in older populations. The age of the subjects fell between 24 years and 101 years, with the interquartile range between the ages of 69 and 83 . Based on the different clinical characteristics of young and old strokes, age was categorized into three groups: young ( $<50$ years of age), middle aged (50-75 years) and older (>75 years), in order to make comparisons more meaningful. Using these categorizations, one can see that just over half of the subjects were in the older age groups (56\%), with $39 \%$ falling in the middle group and a much smaller proportion $(5 \%)$ under the age of 50 years.

The individuals in this study were predominantly white, which is again a reflection of the demographic profile of the surrounding hospital catchment area, although stroke incidence rates have repeatedly been shown to be higher in North American blacks than whites (Gordon, 1993; Kurtzke, 1986). Half of the subjects were married and roughly a third were widowed, again a 
reflection of the higher age distribution of stroke. Marital status was then collapsed into a dichotomous variable with the categories married and not married (including single, widowed, divorced and separated) as a more meaningful variable indicative of a spousal presence. The unknown subjects were not included in this dichotomy under the assumption that they were too few in number to affect the proportions in any substantial way.

Most subjects were living at home on admission, which is similar to the results of other community studies (Bonita, Anderson, \& North, 1987), and likely to be living with family, although one third were living at home alone. The living arrangements variable was also dichotomized to make it more meaningful. Individuals who were living at home or in structured retirement were considered to be similarly autonomous and labelled as living at home. All the individuals receiving some sort of hospital care were labelled as living in an institution. Again the unknown group was omitted from the allocation. It is evident that the overwhelming majority of subjects were living under their own care at the time of hospital admission. Similarly, the "living with" variable was collapsed into living alone or with others. This variable was only applicable to those living outside institutions.

Because of the higher age distribution of stroke, the majority of these subjects were retired from employment at the time of admission, and most of them had high school as their highest level of education attained. And, in keeping with the proportions reported elsewhere in North America (Gordon, 1993; Kurtzke, 1986), the majority of stroke admissions was diagnosed as cerebral infarction, with approximately $14 \%$ categorized as cerebral hemorrhage. Roughly equal numbers of strokes occurred in the left and right hemispheres of the brain, with only a small number of bilateral events. Although some research has suggested that left hemispheric events are more common (Bladin, Smurawska, Alexandrov, Zhu, \& Norris, 1993), this finding was not observed in this sample.

Comorbidity is a common characteristic of older populations, and this was also evident in this study population. One-third of the total group had another illness active along with their stroke at the time of admission. This was most commonly a cardiovascular problem or pneumonia. A relatively large number of subjects also reported a history of musculoskeletal disorders which is to be expected in an older population. Comorbidity is also likely to be seen 
in stroke patients because other illnesses are often risk factors for a stroke, specifically diabetes and cardiovascular disease, and a significant number of our subjects had a history of these complications, particularly cardiovascular disease. Finally, a history of a previous stroke also elevates the risk for a further stroke, with five year recurrence rates reported to be as high as $42 \%$ in male survivors (Kannel, 1971). Almost $30 \%$ of these individuals reported having had a previous stroke prior to their entrance in this study.

Therefore, the stroke admissions from which the participants for the study came, appear to be representative of other stroke populations, with no major differences or anomalies.

\section{ii) Study Participants: Subjects Assessed For Handicap}

Of these 450 consecutive hemispheric stroke patients (436 individuals) admitted to Sunnybrook Health Science Centre, only 164 (36.4\%) were able to participate in the handicap component of this study. One hundred and forty-five $(32.2 \%)$ individuals were assessed for handicap at the three month follow-up period, and $135(30 \%)$ were assessed for handicap at one year post stroke.

\section{Reasons for Non-Participation}

Figure 3 outlines the various reasons why a handicap assessment was not possible at each follow-up period for the majority of strokes in this study. First, as outlined earlier in the statistical analyses section, the first event for one subject who had two strokes was deleted in order to maintain the validity of the statistical assumptions. Ten individuals $(2.2 \%)$ had a second stroke before the three month follow-up assessment, a study endpoint where subjects were reentered into the study as new cases to be assessed for handicap following a different event. Second, $115(25.6 \%)$ individuals had died before the 3 month follow-up period, so could not be assessed for handicap, and $25(5.6 \%)$ of the subjects were simply too ill from their stroke to complete the RNL questionnaire. Similarly, 10 (2.2\%) were too ill from other illnesses to permit a handicap assessment. Third, some subjects could not participate because the handicap measure used in this study requires that subjects have sufficient cognitive and psychological abilities to give accurate responses (Wood-Dauphinee, Opzoomer, Williams, Marchand, \& Spitzer, 1988). 
Twenty-one (4.7\%) were aphasic and could not comprehend the questions in or express their responses to the handicap questionnaire, while 27 (6.0\%) suffered from dementia, such that they could not comprehend the RNL index.

Additionally, a total of 41 individuals (9.1\%) refused to consent to follow-up at three months after their stroke. Furthermore, because the handicap measure is a self-report scale written in the English language, individuals with visual impairments or poor English language skills could not fully participate in the handicap testing, nor could those whose place of residence prevented them from attending follow-ups on a regular basis. Therefore, from a practical standpoint, 13 (2.9\%) subjects had impaired vision (0.6\%) or poor English language skills $(2.2 \%)$ that prevented them from completing the RNL questionnaire. Eighteen (4.0\%) could not return to the hospital outpatient clinic for follow-up, either because they were out of town at the time of their three month follow-up $(3.1 \%)$ or because they were an inpatient at a rehabilitation facility $(0.8 \%)$. An additional nine stroke survivors (2\%) were unable to come into the clinic for follow-up because they found it difficult to leave home due to advanced age.

Finally, four individuals $(0.8 \%)$ could not be located for any follow-up whatsoever. and for six individuals (1.3\%) there was insufficient time in the follow-up visit to complete the RNL questionnaire. (The handicap assessment was part of a much larger follow-up for the overall clinical study, and occasionally there was simply not enough time to complete all assessments.) Additionally, five individuals (1.1\%) who had agreed to participate, subsequently found they could not tolerate the handicap assessment, either because it was too physically demanding after an extensive series of neurocognitive testing or because they found the questions in the RNL too emotional to answer or too personally intrusive.

At the time of the one year follow-up, a further four individuals suffered an additional stroke, bringing the total number of second strokes to 14 (3.1\%), and a further 32 individuals died, bringing the total number of deaths to 147 (32.7\%). A further $27(6.0 \%)$ of the consecutive stroke admissions could not participate at one year because they were simply too ill from their stroke $(4.2 \%)$ or other illnesses $(1.8 \%)$ to complete the handicap questionnaire. Sixteen (3.6\%) were still experiencing speech and comprehension deficits (aphasia), and 24 (5.3\%) were suffering from dementia. 
450 Consecutive Hemispheric Strokes Admitted to Hospital

\section{At 3 Months:}

$1(0.2 \%)$ event for one subject with two strokes was deleted (see statistical analysis section) $145(32.2 \%)$ assessed for handicap $304(67.6 \%)$ not assessed:

$\Rightarrow 208(46.2 \%)$ died or too ill:

$\begin{array}{cl}10(2.2 \%) & \text { re-stroked } \\ 115(25.6 \%) & \text { died } \\ 25(5.6 \%) & \text { too ill from stroke } \\ 10(2.2 \%) & \text { too ill from other illnesses } \\ 21(4.7 \%) & \text { aphasic } \\ 27(6.0 \%) & \text { demented }\end{array}$

$\Rightarrow 96(21.3 \%)$ for other reasons:
$41(9.1 \%)$ refused consent to any follow-up
$3(0.6 \%) \quad$ visual problems
$10(2.2 \%) \quad$ poor English language skills
$14(3.1 \%)$ out of town
$4(0.8 \%)$ in rehab facility
$9(2.0 \%)$ unable to leave home
$6(1.3 \%)$ shortage of time in clinic
$4(0.8 \%) \quad$ could not be located
$5(1.1 \%) \quad$ attempted but could not complete RNL

\section{At 1 Year:}

$135(30.0 \%)$ assessed for handicap at the 1 year follow-up:

$315(70.0 \%)$ not assessed:

- $228(50.7 \%)$ died or too ill:

$\begin{array}{cl}14(3.1 \%) & \text { re-stroked } \\ 147(32.7 \%) & \text { died } \\ 19(4.2 \%) & \text { too ill from stroke } \\ 8(1.8 \%) & \text { too ill from other illnesses } \\ 16(3.6 \%) & \text { aphasic } \\ 24(5.3 \%) & \text { demented }\end{array}$

$-87(19.3 \%)$ for other reasons:

$\begin{aligned} 39(8.6 \%) & \text { refused consent to any follow-up } \\ 2(0.4 \%) & \text { visual problems } \\ 8(1.8 \%) & \text { poor English language skills } \\ 12(2.7 \%) & \text { out of town } \\ 5(1.1 \%) & \text { in nursing home } \\ 12(2.7 \%) & \text { unable to leave home } \\ 2(0.4 \%) & \text { shortage of staff in clinic } \\ 3(0.6 \%) & \text { could not be located } \\ 4(0.8 \%) & \text { attempted but could not complete RNL }\end{aligned}$


Thirty-nine subjects ( $8.6 \%$ of the total group) refused consent to follow-up at one year. Visual and language problems were prohibitive to participation in $10(2.2 \%)$ individuals. Twenty-nine individuals (6.4\%) could not return to the hospital for a handicap assessment either because they were out of town (2.7\%), in a nursing home (1.1\%) or simply unable to leave home because of advanced age (2.7\%). Of those individuals who were able to come in to the clinic for follow-up, $2(0.4 \%$ of the total) did not complete the handicap assessment because one of the neuropsychological testers was unavailable, while $4(0.8 \%)$ found the RNL too physically or emotionally demanding. Three individuals $(0.6 \%)$ could not be located for follow-up.

Therefore, there were essentially two groups of non-participants in this study. The majority were those who could not participate in the handicap assessment either because they had died or because they were too ill to complete the RNL questionnaire. Individuals in the second group were otherwise presumed well enough to consider their handicap situation, but were not assessed due to a shortage of time, geographic, visual or language barriers, or non-consent.

Those who died before the follow-up period are outside the scope of this research project which was to investigate handicap in stroke survivors. However, even if these subjects had survived to the time of the follow-up, it is likely that their handicap would have been significant. For instance, the 115 subjects who died before the three month follow-up lived only a median of eleven days after stroke onset, indicative of the fact that the overwhelming majority $(90 \%$ of those who were assessed) was graded as having had a severe stroke at onset.

For those who were too ill, demented or aphasic to participate, it was not possible to assess handicap in these subjects given the nature of the outcome measure. Because the RNL is a selfreport measure which asks questions pertaining to the consequences of disease in the social context, it is not possible to assess handicap in individuals who are too ill to be participating in the social realm. However, the available data for these ill individuals suggest that it is also likely that their social roles and activities would have been substantially compromised due to their restricted health. To take the three month follow-up period as an example, of the 48 individuals with dementia or aphasia, $71 \%$ were classified as dependent in cognitive ability at three months, while $65 \%$ were classified as dependent or moderately dependent in motor ability. Similariy, for those who could not complete the assessment because they were too ill, the majority (68\%) 
experienced a severe stroke at onset, and $73 \%$ were found to be dependent in motor ability at three months, while $64 \%$ were dependent in cognitive ability.

Therefore, because of the nature of the outcome measure used in this study, handicap could not be assessed in individuals who were severely impaired or confused. Even a surrogate assessment of handicap was not a possible option for these subjects. Because handicap can be such a personal concept, often requiring a value judgement, the validity of a handicap assessment from a surrogate respondent would be questionable. In fact, the correlation between patients' scores on the RNL and those from significant others was not found to be particularly high (.621 to .648) (Wood-Dauphinee, Opzoomer, Williams, Marchand, \& Spitzer, 1988) suggesting that another person's assessment of an individual's handicap may not necessarily reflect that person's own perception. Furthermore, proxy responses have been found to be unreliable in other studies using generic measures of well-being in the elderly (Pierre, Korner-Bitensky, Hanley, \& Wood-Dauphinee, 1995) and other brain injured populations (Sneeuw et al., 1995), particularly in individuals who are more impaired (Sneeuw et al., 1995), for whom proxy information is most needed. Therefore, for the purposes of this study, it was only possible to include those subjects who were well enough to make a meaningful report of their handicap situation. This has been the practice in other similar studies (Ahlsio, Britton, Murray, \& Theorell, 1984).

While the original intent of this research was to investigate the determinants of handicap in stroke survivors, the objective now becomes more refined, namely, to determine the effects of impairments and disabilities on handicap in hemispheric stroke survivors who are well enough to complete a handicap questionnaire. Therefore, of the $\mathbf{4 5 0}$ consecutive hemispheric stroke patients who were admitted to Sunnybrook Hospital, 201 (44.7\%) subjects were excluded from the eligible pool of admissions because they had died or were too ill at the time of follow-up (208 subjects at three months 228 at one year), and one stroke admission was excluded because of duplicate data. This brings the eligible total to 248 (55.1\%) subjects who were well enough to be assessed for handicap throughout the recovery period: $241(53.6 \%)$ at three months post stroke and $222(49.3 \%)$ at one year. All findings from this research apply only to these subset of subjects. 
While this may seem a radical departure from the original intent of this thesis, it is. nevertheless, inevitable in this type of research. In fact, handicap may only really be assessable in individuals who are living everyday life (Badley, 1987). However, this points to one of the defining characteristics of this type of research: individuals who are able to complete a handicap assessment tend to be less ill than those who cannot, and any analysis of handicap is therefore limited to subjects who are in better health. And indeed, when comparing the characteristics of the ill subjects $(n=201)$ with those who were well enough to be assessed $(n=248)$, there were statistically significant differences in health and independence between the two groups.

The subjects who died or who were too ill to be assessed were statistically significantly more likely to be older than those who could be followed $(77.7 \pm 9.6$ years vs $72.4 \pm 12.8$ years, respectively, $t_{443.5}=5.01, p<0.0001$ ), and they were also far more likely to have had a severe stroke $\left(74.4 \%\right.$ vs $15.4 \%$, respectively, $\left.\chi_{2}^{2}=110.9, p<0.0001\right)$. Comorbidity was also more common in non-assessable subjects at onset $(41.5 \%)$ than assessable subjects $(26 \%)\left(\chi_{1}^{2}=12.0\right.$, $p<0.0001)$, as was a history of previous stroke $\left(41.6 \%\right.$ vs $19.8 \%$, respectively, $\chi_{1}^{2}=25.0$, $\mathrm{p}<0.0001)$. More of the assessable subjects were independent in motor ability $(94.3 \%$ vs $\mathbf{7 0 . 8 \% )}$ and cognitive ability (94.7\% vs $68.5 \%$ ) before their stroke compared to non-assessable subjects $(p<0.0001)$. Non-assessable subjects were also less likely to have been living at home before their stroke compared to assessable subjects $\left(90.4 \%\right.$ vs $98.4 \%$, respectively, $\chi_{1}^{2}=14.3$, $\mathrm{p}<0.0001$ ). Non-assessable subjects were also statistically more likely to have had a hemispheric hemorrhage than an infarct $\left(19.4 \%\right.$ vs $\left.9.3 \%, \chi_{1}^{2}=9.6, p=0.002\right)$, indicative of the higher mortality in hemorrhagic events (Kelly-Hayes, 1990; Sacco, Wolf, Kannel, \& McNamara, 1982; Chambers, Norris, Shurvel, \& Hachinski, 1987). Finally, assessable subjects reported a statistically better quality of life in the week preceding their stroke than non-assessable subjects $\left(8.6 \pm 1.6\right.$ vs $6.8 \pm 2.4$ on the Spitzer Quality of Life Index, respectively, $\left.t_{141}=6.5, p<0.0001\right)$

Therefore, by excluding subjects who died or who were severely ill, the determinants of handicap can only be assessed in subjects who are in better health, and this must be kept in mind when considering the generalizability of the results. Some strategies have been proposed to deal with such losses to follow-up in health outcome studies (Diehr et al., 1995). These range from removing the dead from all analyses to assigning them the worst possible score on the outcome 
measure. For instance, the non-assessed subjects in this study could have been assigned a score of zero on the RNL index since it is evident from their characteristics that even if they could have been assessed, it is likely that their propensity for extensive disabilities and impairments would have led to a severe restriction in life activities and consequently greater handicap. Such strategies may have merit when the outcome of interest is health status and the purpose of the study is to investigate the impact of health-related interventions. But for this project. where the outcome of interest is a socially constructed state of being, it is difficult to speculate about the level of handicap in the dead or ill with any degree of certainty. Instead, it is preferable to limit the scope of the study to those for whom handicap can be a measurable construct.

One of the advantages of restricting the eligible population to those who are well enough, is that it can improve the internal validity of the comparison of exposed and unexposed subjects. Specifically, by excluding subjects who are too ill from other conditions, we are equalizing the opportunity for handicap amongst participants apart from their stroke-induced impairments and disabilities. By excluding, for instance, subjects who are too ill from cancer or other comorbid conditions, it is possible to get a better estimate of the effects of the stroke-induced impairment and disability on handicap that is not contaminated by the effects of other illnesses.

But it should also be noted from Figure 3 that not all of the 248 subjects who were well enough to be followed completed a handicap assessment, although it is presumed that they could have. Only $164(66.1 \%)$ completed a handicap assessment at either one of the two follow-up periods, while $84(33.9 \%)$ did not due to other reasons. Of the 241 survivors who were well enough to participate at three months post-stroke, $96(39.8 \%)$ were not assessed because they were unavailable or refused to come in to the clinic for follow-up, or because of time shortages, visual or language problems (see Figure 3). Similarly, of the 222 survivors who were well enough to be assessed for handicap at one year, 87 (39.2\%) were not assessed for similar reasons.

The tables in Appendix 9 compare the characteristics of these participants and nonparticipants according to their characteristics at stroke onset, and indicate that these two groups of subjects were generally similar with respect to their demographic and clinical profile. Roughly equal proportions were male, and they were equally likely to be married and to be living at home at the time of admission. Prior to their stroke, equivalent proportions of 
participants and non-participants were independent in motor ability and cognitive ability. Comparable proportions were admitted with other comorbid conditions at stroke onset, and. although not all subjects received an impairment assessment (in part due to the same reasons that prevented a handicap assessment), similar proportions of those who were assessed were graded as having a mild, moderate or severe stroke.

However, there were differences between the participants and non-participants with respect to age, type of stroke, pre-stroke quality of life and history of diabetes. Participants were more likely to be younger than non-participants (mean age of $70.9=13.2$ years vs $75.4 \pm 11.6$ years, respectively, $\mathrm{t}_{246}=2.7, \mathrm{p}<0.006$ ) and they were more likely to have had a hemorrhage than the non-participants ( $12.2 \%$ vs $3.6 \%$, respectively, $\chi^{2}{ }_{1}=4.91, p<0.03$ ). Participants also reported a better quality of life in the week preceding their stroke than did non-participants (mean Spitzer score of $8.8 \pm 1.6$ vs $8.1 \pm 1.7$, respectively, $t_{217}=2.55, p<0.01$ ). However, non-participants were more likely to have had a history of diabetes than the participants ( $25 \%$ vs $11.6 \%$, respectively, $\chi_{1}^{2}=7.52, p<0.006$ ). These differences may have effects on the external and internal validity of the study findings, and will be explored later in the discussion section. 


\section{BIVARIATE ANALYSES}

The first-level relationships between handicap and the independent variables were assessed with bivariate analyses. The assumptions underlying these statistical tests were first verified using exploratory data analysis. In the first place, a statistical test comparing means requires that the values of the dependent variable be normally distributed within each group of the independent categorical variable, although these tests are robust with large sample sizes. This assumption was examined using exploratory box plots, and the data were found to be roughly normally distributed for each of the groups. Secondly, with respect to the assumption of equal variances within each comparison group, exploratory analyses revealed that the variances were roughly equal in each group of the independent categorical variable. Where the variances showed some difference in categorical variables with only two groups, t-tests for unequal variances were used. Where there was uncertainty as to the equality of variances in categorical variables with more than two groups, Bartlett's Test for the Homogeneity of Variances (Rosner, 1990) was performed (although analysis of variance is robust with respect to moderate departures from this assumption (Boneau, 1960)). Finally, the observations for these analyses were obtained from independently measured subjects (except for one of the subjects with a second stroke, and this information was dropped - see Statistical Analysis Strategies), satisfying the assumption of independent observations.

Following the verification of the statistical assumptions, bivariate analyses were then performed. For independent categorical variables with two groups, t-tests were performed; oneway analysis of variance was used to compare RNL means in independent categorical variables with more than two categories. Simple linear regression was used to assess the association of RNL scores with a continuous independent variable. When looking at these simple bivariate relationships between stroke handicap and the independent variables of interest, a number of different patterns emerged.

\section{i) Handicap By Stroke Impairment}

First, Table 1 shows the average RNL scores for individuals with different levels of stroke impairment as measured by the Adams' Hemispheric Stroke Scale. At three months after stroke 
onset, $78 \%$ of the 116 individuals who were assessed for impairment were graded as having a mild impairment, 20\% were moderately impaired and $2 \%$ had severe stroke impairment. Twenty-nine $(20 \%)$ of the 145 individuals assessed for handicap at three months were not assessed for impairment, with an average handicap score of $74.8 \pm 22.0^{6}$. Nine months later. similar proportions were observed. Eighty percent were assessed as having mild stroke impairment, $18 \%$ were moderately impaired and $2 \%$ were severely impaired, indicative of the fact that little neurological recovery takes place after 3 months (Andrews, Brocklehurst, Richards, \& Laycock, 1981; Partridge, Johnston, \& Edwards, 1987; Skilbeck, Wade, Hewer, \& Wood, 1983). Thirty-eight (28\%) of the 135 subjects assessed for handicap at one year were not assessed for impairment, with a mean RNL score of $84.8 \pm 13.0$ (again see footnote 6).

Table 1

Average RNL Scores ( \pm s.d.) By Level of Impairment

\begin{tabular}{|c|c|c|}
\hline $\begin{array}{l}\text { Level of Stroke Impairment } \\
\text { at Time of Follow-Up }\end{array}$ & 3 Month RNL & One Year RNL \\
\hline Mild & $\begin{array}{c}79.3 \pm 17.6 \# \\
(n=90)\end{array}$ & $\begin{array}{c}82.5 \pm 15.7 \# \\
(n=78)\end{array}$ \\
\hline Moderate & $\begin{array}{c}51.4 \pm 22.3 \\
(n=23)\end{array}$ & $\begin{array}{c}61.1 \pm 22.6 \\
(n=17)\end{array}$ \\
\hline Severe & $\begin{array}{c}10.4 \Omega 18.1 \\
(n=3)\end{array}$ & $\begin{array}{c}47.5 \pm 8.6 \\
(n=2)\end{array}$ \\
\hline
\end{tabular}

\# differences between subgroups $\mathrm{p}<0.0001$

It is clear from Table 1 that at both the three month and one year follow-up periods, individuals with a more mild stroke impairment at the time of follow-up reported less handicap. A high RNL score (up to a maximum of 100) indicates minimal handicap, while a low score (minimum of 0 ) indicates greater handicap. RNL scores decreased in individuals with more

\footnotetext{
${ }^{6}$ It appears that these subjects may, in fact, have been assessed, but that their data were subsequently mislaid due to a data entry oversight. While the data are in the process of being retrieved, complete files were not available at the time of this writing. However, there is no reason to suspect that the loss of these data was anything but a random occurrence without any systematic pattern, as indicated by the fact that the average handicap score for these missing subjects is similar to that for subjects with complete impairment data (mean RNL of $72.2 \pm 23.8$ at 3 months, $78.0 \pm 19.2$ at one year).
} 
severe stroke impairment, suggesting that handicap increases as impairment increases. All these mean RNL scores were statistically significantly different from each other as revealed by one way analysis of variance with post-hoc comparisons (Tukey studentized range test) at 3 months $\left(F_{2.113}=37.5, p<0.0001\right)$ and at one year $\left(F_{2.94}=14.28, p<0.0001\right)$.

Similarly, those individuals with greater psychological impairment after their stroke also reported greater handicap. Table 2 gives the average RNL scores for individuals who were classified as depressed according to the Zung Depression Scale. Roughly $20 \%$ of the individuals who were assessed for handicap either at three months or at one year experienced some form of dysphoric mood disorder after their stroke onset. These individuals reported a statistically significantly greater handicap than those without psychological impairment $\left(t_{33.3}=5.5, p<0.0001\right.$ at 3 months; $t_{127}=3.34, p<0.001$ at one year). Only a small number of subjects were not assessed for depression at the two follow-up periods, making it unlikely that they would change the results substantially if their mood state had been known.

Table 2

Average RNL Scores ( \pm s.d.) By Depression

\begin{tabular}{|c|c|c|}
\hline $\begin{array}{c}\text { Depression Present at Follow- } \\
\text { Up }\end{array}$ & 3 Month RNL & One Year RNL \\
\hline No & $\begin{array}{c}79.7 \pm 16.3 \# \\
(\mathrm{n}=112)\end{array}$ & $\begin{array}{c}83.0 \pm 15.6 \# \\
(\mathrm{n}=105)\end{array}$ \\
\hline Yes & $\begin{array}{c}53.1 \pm 24.3 \\
(\mathrm{n}=28)\end{array}$ & $\begin{array}{c}71.2 \pm 19.4 \\
(\mathrm{n}=26)\end{array}$ \\
\hline
\end{tabular}

\# differences between subgroups $p<0.001$

\section{ii) Handicap by Stroke Disability}

Handicap also appears to increase with disability. Table 3 gives the average RNL scores for individuals rated at different levels of disability according to the FIM at the two follow-up periods. At three months post-stroke, $83 \%(n=120)$ of the assessed subjects were independent in motor ability, $14 \%(n=20)$ were moderately dependent and $3 \%(n=5)$ were dependent in mobility. This is a higher proportion of independent survivors at three months than reported in other hospital studies (Kotila, Waltimo, \& Marjaiisa, 1984), no doubt due to the better clinical 
and demographic profile of the subjects assessed for handicap. The overwhelming majority of these individuals were independent in cognition at three months $(88 \%)$, and again independent in motor ability (83\%) and cognition $(87 \%)$ at one year.

Table 3

Average RNL Scores ( \pm s.d.) By Level of Disability

\begin{tabular}{|c|c|c|}
\hline $\begin{array}{l}\text { Level of Disability at Time of } \\
\text { Follow-Up }\end{array}$ & 3 Month RNL & One Year RNL \\
\hline \multicolumn{3}{|c|}{ Mobility Dimension } \\
\hline Independent & $\begin{array}{c}78.4 \odot 18.1 \# \\
(n=120)\end{array}$ & $\begin{array}{c}83.9 \pm 14.9 \# \\
(n=112)\end{array}$ \\
\hline Moderately Dependent & $\begin{array}{c}51.9 \pm 21.3 \\
(n=20)\end{array}$ & $\begin{array}{c}61.8 \pm 18.4 \\
(n=21)\end{array}$ \\
\hline Dependent & $\begin{array}{c}14.3 \pm 19.9 \\
(n=5)\end{array}$ & $\begin{array}{c}46.5 \pm 25.7 \\
(n=2)\end{array}$ \\
\hline \multicolumn{3}{|c|}{ Cognition Dimension } \\
\hline Independent & $\begin{array}{c}75.4 \pm 20.9 \# \\
(n=128)\end{array}$ & $\begin{array}{c}83.0 \pm 15.8 \# \\
(n=117)\end{array}$ \\
\hline Moderately Dependent & $\begin{array}{c}62.0 \pm 19.8 \\
(n=14)\end{array}$ & $\begin{array}{c}62.7 \pm 18.2 \\
(n=15)\end{array}$ \\
\hline Dependent & $\begin{array}{c}0.0 \pm 0 \\
(n=3)\end{array}$ & $\begin{array}{c}45.5 \pm 7.3 \\
(n=3)\end{array}$ \\
\hline
\end{tabular}

\# differences between subgroups $p<0.0001$

It is evident from Table 3 that those individuals who were more independent in the mobility and cognition dimensions of the FIM reported statistically significantly higher RNL scores (less handicap) than those who were more dependent. One way analysis of variance revealed that the mean RNL scores in the three mobility groups were statistically different at three months $\left(\mathrm{F}_{2.142}\right.$ $=42.68, \mathrm{p}<0.0001$ ) and post-hoc analyses revealed that the mean $\mathrm{RNL}$ scores in all three disability levels were different from each other. At one year post-stroke, analysis of variance showed that there were differences in the mean RNL scores between the three mobility levels $\left(F_{2.132}=22.63, p<0.0001\right)$ and post-hoc analyses revealed that the differences were between the dependent and independent groups, and between the moderately dependent and independent 
groups, but no significant difference was detected between the RNL means in the dependent and moderately dependent groups, most likely due to the smaller number of subjects in the dependent groups.

When comparing handicap scores across the cognition dimension of the FIM, the mean RNL scores were found to be significantly different between all cognitive disability groups at three months $\left(\mathrm{F}_{2.142}=21.59, \mathrm{p}<0.0001\right)$ with post-hoc comparisons indicating that the differences existed between all subgroups, except the independent and moderately dependent subgroups. For the one year follow-up period, there was a statistically significant difference between all three cognitive disability levels $\left(F_{2.132}=17.84, p<0.0001\right)$ with post-hoc tests indicating that the differences were between those who were dependent and independent in cognition, but not between those who were classified as dependent and moderately dependent at one year.

Therefore, more severe impairments and disabilities do appear to increase handicap following stroke. But what else might be influencing this apparent relationship?

\section{iii) Handicap by Environmental Factors}

A number of different environmental factors were postulated to influence whether impairments and disabilities become handicaps following stroke. First, family structure, that is marital status and living arrangements, were hypothesized to be a potentially important source of external support which could act to minimize handicap. When looking at the average RNL scores in people who were married and not married (single, widowed, separated or divorced), we found that handicap scores was similar in individuals who were married $(n=79$, mean $R N L$ $=73.5 \pm 22.4)$ and not married $(n=66$, average $R N L$ score at three months $=71.5 \pm 24.7)$, with no statistically significant difference between the two groups $(p=0.6)$. We also found that people living in an institution $(\mathrm{n}=27)$ at three months had significantly more handicap than those living at home $(n=116)$ (mean RNL scores of $47.0 \pm 26.3$ vs $79.2=17.3$, respectively; $t_{31.4}=6.1$, $\mathrm{p}<0.0001$, two tailed t-test for unequal variances), although this could of course be related to the underlying level of impairment or disability. Surprisingly, when looking at living arrangements for those living at home at three months, we found that people who were living alone $(n=39)$ reported no statistically significant difference in handicap (average 3 month RNL score of 80.0 
\pm 18.2 ) to those who were living with either family or friends ( $n=75$, mean $R N L=78.5 \pm 17.0$ ) $(p=0.6)$. This finding may be related to the underlying level of disability since individuals with less disability may report less handicap and may be more able to live at home alone. These findings did not differ for the one year follow-up period.

Wealth (SES), as measured by level of education obtained, was also postulated to be an important environmental resource influencing post-stroke handicap. However, there was no statistically significant difference between RNL scores in individuals with different levels of education. Health and social service resources were also postulated to be important environmental factors with the potential to influence the handicap creation process. The use of rehabilitation therapy was particularly thought to be important for stroke survivors. In this study the use of rehabilitation services included either outpatient or inpatient therapy. Our results show that survivors who had some form of rehabilitation therapy before the three month followup visit reported significantly lower RNL scores (greater handicap) than those who did not have rehab $(p<0.001$ ) (Table 4). Similarly, individuals who received rehabilitation therapy between the three month and one year visits also reported significantly greater handicap at one year $(p<0.002)$.

Table 4

Average RNL Scores ( \pm s.d.) by Use of Rehabilitation Therapy

\begin{tabular}{|c|c|c|}
\hline $\begin{array}{c}\text { Use of Rehab Prior to } \\
\text { Follow-Up }\end{array}$ & 3 Month RNL & 1 Year RNL \\
\hline No & $\begin{array}{c}85.4 \pm 15.8 \# \\
(\mathrm{n}=41)\end{array}$ & $\begin{array}{c}85.9 \pm 12.8^{*} \\
(\mathrm{n}=49)\end{array}$ \\
\hline Yes & $\begin{array}{c}70.7 \pm 19.7 \\
(\mathrm{n}=80)\end{array}$ & $\begin{array}{c}75.6 \pm 20.4 \\
(\mathrm{n}=71)\end{array}$ \\
\hline
\end{tabular}

* differences between subgroups $p<0.002$

\# differences between subgroups $\mathrm{p}<0.001$

The reasons for this finding is likely due to the underlying level of disability, since individuals with more disability are more likely to be in need of rehabilitation therapy, and also more likely to have more handicap. This suggests an interaction between the benefits of 
rehabilitation therapy on handicap at different levels of ability, which needs to be examined further in a multivariate analysis.

\section{iv) Handicap by Pre-Stroke Disability}

The results suggest that post-stroke disability affects handicap, but how much of this disability was actually present before the stroke? In this case, it is not the stroke-induced disability that is important, but disability related to other factors. Table 5 shows the average handicap scores at three months and one year for individuals who reported different levels of prestroke ability.

Table 5

Average RNL Scores (t s.d.) By Level of Pre-Stroke Disability

\begin{tabular}{|l|c|c|}
\hline \multicolumn{3}{|c|}{ Mobility Dimension } \\
\hline & $\begin{array}{c}74.5 \pm 21.0 \# \\
(\mathrm{n}=138)\end{array}$ & $\begin{array}{c}80.3 \pm 17.9 \\
(\mathrm{n}=131)\end{array}$ \\
\hline Independent & $\begin{array}{c}39.7 \pm 35.1 \\
(\mathrm{n}=6)\end{array}$ & $\begin{array}{c}68.2 \pm 17.0 \\
(\mathrm{n}=4)\end{array}$ \\
\hline Moderately Dependent & $0 \pm 0$ & - \\
& $(\mathrm{n}=1)$ & Year RNL \\
\hline Dependent & Cognition Dimension \\
\hline & $\begin{array}{c}74.4 \pm 21.0 \# \\
(\mathrm{n}=137)\end{array}$ & $\begin{array}{c}80.2 \pm 17.9 \\
(\mathrm{n}=130)\end{array}$ \\
\hline Independent & $\begin{array}{c}42.8 \pm 44.4 \\
(\mathrm{n}=5)\end{array}$ & $\begin{array}{c}76.4 \pm 20.1 \\
(\mathrm{n}=3)\end{array}$ \\
\hline Moderately Dependent & $26.8 \bullet 37.8$ & $53.5 \pm 0$ \\
& $(\mathrm{n}=2)$ & $(\mathrm{n}=1)$ \\
\hline Dependent &
\end{tabular}

\# differences between subgroups $p<0.0001$

Again, we see that individuals who were more independent in both the mobility and cognition dimensions of the FIM before their stroke had higher RNL scores, or lower handicap, at both the three month and one year follow-up periods. The mean handicap scores at three months were 
significantly different from each other in both the pre-stroke motor and cognitive disability subgroups $\left(F_{2.142}=13.07, p<0.0001\right.$ for motor disability; $F_{2.141}=9.20, p<0.0002$ for cognitive disability), with the differences lying between all groups except the moderately dependent and dependent groups, most likely due to the small number of cases in the dependent groups. However, at one year after stroke onset, the mean handicap scores did not differ across levels of pre-stroke disability $\left(F_{1.133}=1.78, p=0.2\right.$ for motor disability; $F_{2.131}=1.15, p=0.3$ for cognitive disability), suggesting that pre-stroke disability may only be important to handicap in the more immediate period following stroke, but not in the longer term.

So the previously observed relationship between post-stroke disability and post-stroke handicap at three months may, in fact, be due to the level of disability present before the stroke. In order to tease out the relative importance of these two variables, multivariate analyses are necessary.

\section{v) Handicap by Other Comorbid Conditions}

The literature review also suggested that other comorbid conditions could be important determinants of handicap, apart from the disabilities and impairments associated with stroke. When looking at the handicap scores in individuals who did and did not have other comorbid conditions we see some surprising findings.

First, there was no difference in handicap between those individuals who came into hospital with other illnesses at the time of their stroke onset. Although the RNL scores were higher (less handicap) at both the three month and one year follow-up periods for those who did not present with concomitant illnesses, the differences were not statistically significant $(p=0.10$ at 3 months; $\mathrm{p}=0.30$ at 1 year). Furthermore, individuals with a history of previous strokes did not report significantly greater handicap than those with no stroke history $(p=0.56$ at 3 months; $p=0.27$ at one year). Similarly, a history of diabetes, cardiovascular disease, cancer or musculoskeletal problems did not seem to affect handicap throughout the follow-up period.

However, when comparing handicap scores in individuals who had other stroke-related illnesses occurring in the intervening period between onset and the three month follow-up visit, we found that survivors with stroke related intercurrent illnesses $(n=46)$ had significantly greater 
handicap than those without $(n=97)$ (average three month RNL scores of $63.0 \pm 27.6$ vs $77.9 \pm$ 18.8, respectively, $\mathrm{p}<0.001$ ). This trend was observed again at the one year follow-up visit, although the difference was not statistically significant (average RNL scores of $77.3 \pm 19.3$ vs $82.8 \pm 15.8, \mathrm{p}=0.08$ ). However, these differences were not observed when comparing handicap scores in individuals with non-stroke related intercurrent illnesses.

\section{vi) Handicap by Other Factors}

Side and type of stroke were also thought to be potential determinants of handicap. However, individuals with left hemispheric strokes showed no difference in handicap throughout the follow-up period from those with right hemispheric events or bilateral lesions. Similarly, handicap did not differ significantly between hemorrhages or infarcts, nor did it vary by the sex of the subject.

Pre-stroke quality of life was also thought to be a potential factor influencing post-stroke handicap. We found that at both the three month and one year follow-up periods, there was some indication that a better quality of life prior to the stroke was associated with less handicap after the stroke (Table 6). When plotting the pre-stroke Spitzer scores against the RNL scores at both three months and one year (data not shown), a loose positive straight line relationship was evident with higher Spitzer scores associated with higher RNL scores.

\section{Table 6}

Simple Linear Regression Between

Pre-Stroke Quality of Life and Post-Stroke Handicap

\begin{tabular}{|c|c|c|}
\hline & Beta Estimate & Associated Probability \\
\hline 3 Month Follow-Up & 4.71 & 0.0001 \\
\hline I Year Follow-Up & 2.70 & 0.04 \\
\hline
\end{tabular}

Interestingly, age also appeared to be an important determinant of post-stroke handicap. At both the three month and one year follow-up visits, handicap increased as age increased. Individuals in the oldest age group reported greater handicap than those in the two younger groups (Table 7). These means were significantly different (one-way analysis of variance at three months, $\left.F_{2.142}=3.53, p<0.03\right)\left(F_{2.132}=4.9, p<0.009\right.$ at one year). This pattern could of 
course be due to the fact that older individuals may also be at greater risk for disability. so these relationships need to be clarified further in multivariate analyses.

Table 7

Average RNL scores ( \pm s.d.) by Age Group

\begin{tabular}{|c|c|c|}
\hline Age Group & 3 Monih RNL & 1 Year RNL \\
\hline$<50$ years & $\begin{array}{c}76.2 \pm 26.1 \bullet \\
(n=15)\end{array}$ & $\begin{array}{c}82.0=23.1 \# \\
(n=14)\end{array}$ \\
\hline $50-75$ years & $\begin{array}{c}77.3 \pm 20.1 \\
(n=65)\end{array}$ & $\begin{array}{c}84.4 \pm 15.5 \\
(n=63)\end{array}$ \\
\hline$>75$ years & $\begin{array}{c}66.9 \pm 24.9 \\
(n=65)\end{array}$ & $\begin{array}{c}74.6 \pm 17.8 \\
(n=58)\end{array}$ \\
\hline
\end{tabular}

* differences between subgroups $\mathrm{p}<0.05$

\# differences between subgroups $p<0.01$

\section{vii) Summary}

So we have seen that stroke-induced impairments and disabilities do appear to increase the likelihood of handicap in stroke survivors, lending support to the conceptual model of the ICIDH. However, we have also seen that post-stroke handicap was also influenced by pre-stroke level of ability along with age, place of residence, use of rehabilitation therapy, pre-stroke quality of life and intercurrent illnesses. Therefore, in order to disentangle the possible effects of other factors and determine the real impact of stoke-induced impairments and disabilities on poststroke handicap, it was necessary to perform multivariate analyses. 


\section{MULTIVARIATE ANALYSES}

In order to determine which variables influence post-stroke handicap after controlling for the effects of other factors, a multi-way analysis of variance was performed for each of the two follow-up time periods. Although some of the potential predictive variables did not appear to influence handicap in the bivariate analyses, all variables of interest were included again in the multivariate analyses, to make sure that no relationships were being masked or disguised by other variables in the bivariate comparisons. The assumptions underlying analysis of variance were validated as outlined in the bivariate analyses.

\section{i) At Three Months Post-Stroke}

One hundred and forty-five subjects were assessed for handicap at the three month follow-up period. To begin with, the main independent variables of interest, impairment and disability, were added in turn to an analysis of variance statistical model to see which ones were important determinants of handicap at three months post-stroke. We saw in the bivariate analyses that both stroke impairment, as measured by the Adams' Stroke Scale, and motor disability were associated with handicap on their own. But when these two variables were added to the model together, stroke severity was no longer significant $\left(F_{2.111}=2.49, p=0.092\right)$ although motor disability was $\left(F_{2,111}=5.81, p<0.004\right)$. This is probably because motor ability and stroke severity are highly correlated. The addition of the mobility variable to the model with the impairment variable already present reduced the significance of the effect of impairment and increased the standard errors of the estimates of both mobility and severity. Therefore, since these two variables are highly correlated only one of these two independent variables could be included in the statistical model, and motor disability was statistically significantly associated with handicap over and above the effects of impaiment.

When the cognitive disability variable was then added to the model with only the motor disability variable present, it remained significant $\left(F_{2.140}=4.17, p<0.017\right)$, with post-hoc comparisons indicating that differences existed between the RNL scores of all subgroups in both variables. Together the mobility and cognition disability variables explained $41 \%\left(R^{2}=0.411\right)$ of the variance in handicap at three months post-stroke (Table 8). 
Table 8

Multi-Way Analysis of Variance

Motor Ability and Cognitive Ability in Association with 3 Month RNL

$R^{2}=0.411 \quad F_{4,140}=24.38 \quad p<0.0001$

$\begin{array}{llll}\text { Variable } & \text { df } & \mathrm{BV} \text { Value } & \mathrm{F}\end{array}$

$\begin{array}{llll}\text { Motor Ability } & 2 & 21.07 & 0.0001\end{array}$

$\begin{array}{llll}\text { Cognitive Ability } & 2 & 4.17 & 0.0173\end{array}$

When assessing the additional effects of adding the psychological impairment variable to the model with the two disability variables already present, analysis of variance revealed that the cognition variable was no longer significant when the depression variable was included in the model, although motor disability remained important (Table 9).

Table 9

Multi-Way Analysis of Variance

Motor Ability, Cognitive Ability and Depression in Association with 3 Month RNL

$\mathrm{R}^{2}=0.414 \quad \mathrm{~F}_{4,135}=23.89 \quad \mathrm{p}<0.0001$

$\begin{array}{llll}\text { Variable } & \text { df } & \mathrm{EV} \text { Value } & \mathrm{Br}\end{array}$

$\begin{array}{llll}\text { Motor Ability } & 2 & 16.09 & 0.0001\end{array}$

$\begin{array}{llll}\text { Cognitive Ability } & 1 & 2.01 & 0.1588\end{array}$

$\begin{array}{llll}\text { Depression } & 1 & 38.48 & 0.0001\end{array}$

When the cognitive ability variable was then removed from the model, motor ability and depression together explained $41 \%$ of the variance in handicap at three months post-stroke (Table 10).

Table 10

Multi-Way Analysis of Variance

The Association of Motor Ability and Depression with 3 Month RNL

$\mathrm{R}^{2}=0.4057 \quad \mathrm{~F}_{3.136}=30.95 \quad \mathrm{p}<0.0001$

Variable

df

E Vaiue

$\mathrm{Pr}>\mathrm{F}$

Motor Ability

$2 \quad 16.89$

0.0001

Depression

$1 \quad 41.14$

0.0001 
These two variables, motor ability and depression were then kept in the model as key components of the primary hypothesis, and the other potentially important variables were added to the model in turn to see if they were additionally associated with handicap, or to see if they changed any of the relationships already identified.

In these series of analyses, pre-stroke ability, a history of stroke, comorbid conditions. intercurrent illnesses (both stroke related and non-stroke related), marital status, gender, age. side or type of stroke, level of education, and pre-stroke quality of life were not important in predicting handicap, over and above motor disability and post-stroke depression. Furthermore, when age and pre-stroke disability were added in turn to the model with the significant impairment and disability variables already present, neither one changed the effects of the independent variables, nor did they raise the standard errors of their estimates. This was also true for stroke history and comorbid conditions. Therefore, a subject's age, pre-morbid disability, stroke history or comorbid conditions are not confounders and are not the underlying reasons for the observed relationship between motor disability, depression and post-stroke handicap. (These effects did not vary depending on whether age was included as a categorical or continuous variable.) Additionally, neither of these variables added anything extra to the model on their own, suggesting that age, pre-stroke disability, stroke history and comorbidity are not independently associated with handicap in the subacute stage over and above level of mobility and depression.

However, two environmental factors, living arrangements at three months and use of rehabilitation therapy prior to the three month follow-up period were found to be associated with handicap over and above motor ability and depression (Table 11). When living arrangements was added to the model with only motor ability and depression, it remained a statistically significant, with those individuals living at home reporting a statistically higher RNL score (lower handicap) than those living in institutions, when controlling for level of motor ability and psychological impairment (adjusted least-squares RNL means of 60.8 vs 46.8, respectively). This suggests that there may be some benefit to living at home over an institutional setting across individuals with similar disability and impairment levels, although this may also be an artifact of the RNL index items and how they are scored by people in different settings. Individuals in 
an institutional setting may not have the same opportunities for social participation and interaction as those living in the community, and this may artificially lower their RNL scores by virtue of their limited opportunities. This finding should, therefore, be interpreted with caution. Following the rationale for the interaction effects of environmental factors on handicap, multiplicative interaction terms combining living arrangements with motor ability and depression were added to the model to see if the benefits of living arrangements varied by level of impairment and disability. These interaction terms were not found to be statistically significant $(p<0.15)$.

Table 11

Multi-Way Analysis of Variance

Final Model Predicting 3 Month RNL

$R^{2}=0.421 \quad F_{s .111}=16.11 \quad p<0.0001$

$\begin{array}{lll}\text { Variable } & \text { df } & \text { E Value }>E\end{array}$

$\begin{array}{llll}\text { Motor Ability } & 2 & 3.85 & 0.0240\end{array}$

$\begin{array}{llll}\text { Depression } & 1 & 23.09 & 0.0001\end{array}$

$\begin{array}{llll}\text { Living Arrangements } & 1 & 7.03 & 0.0092\end{array}$

$\begin{array}{llll}\text { Rehabilitation Therapy } & 1 & 9.61 & 0.0025\end{array}$

When examining the effects of external resources such as rehabilitation therapy, this was also found to be statistically significantly associated with handicap when adjusting for the effects of motor ability, depression and living arrangements $(p<0.003)$. When examining the least-squares adjusted means, we found that individuals who received therapy reported greater handicap (mean RNL score of 53.1) than those who did not (mean RNL score of 61.8). As discussed in the bivariate analyses, this is counterintuitive to what one might expect. It was thought that rehabilitation therapy would be of benefit to the lives of survivors, not a hindrance. In order to better understand this puzzling finding, we examined the mean handicap scores broken down by level of motor ability and use of rehabilitation therapy (Table 12).

One can see from Table 12 that the overall mean RNL scores in the therapy group is reduced by the low RNL score for those individuals who are dependent in mobility. One can also see that rehabilitation services appear to facilitate a reduction in handicap for those who are moderately 
dependent but not for those who are independent in mobility. Therefore, the greater overall handicap score observed in those individuals who had therapy is likely due to an underlying propensity for individuals with disabilities to have therapy. But when examining the effects of rehabilitation therapy on handicap by separate disability groups, the hypothesized benefit of rehabilitation does, in fact, exist in those who have more dependence in mobility. This makes sense, since the benefits of therapy will vary depending on the needs of the individuals who receive it.

\section{Table 12}

Average RNL Scores ( \pm s.d.) by Rehabilitation Therapy and Motor Ability

\begin{tabular}{|c|c|c|}
\hline \multirow[b]{3}{*}{ Motor Ability } & \multicolumn{2}{|c|}{ Use of Rehabilitation Therapy before 3 Months } \\
\hline & Yes & No \\
\hline & & \\
\hline Independent & $\begin{array}{c}74.6 \pm 16.6 \\
(n=64)\end{array}$ & $\begin{array}{c}87.8 \pm 11.2 \\
(n=39)\end{array}$ \\
\hline $\begin{array}{l}\text { Moderately } \\
\text { Dependent }\end{array}$ & $\begin{array}{c}59.9 \pm 19.3 \\
(n=14)\end{array}$ & $\begin{array}{c}37.9 \pm 20.7 \\
(n=2)\end{array}$ \\
\hline Dependent & $\begin{array}{c}20.2 \bullet 28.6 \\
(n=2)\end{array}$ & - \\
\hline
\end{tabular}

These results suggest the presence of an interaction effect between level of disability and use of rehabilitation therapy, and indeed such an interaction term was statistically significant when added to the model $\left(F_{1,110}=5.97, p<0.02\right)$. However, the numbers in two of the five cells in Table 12 are small $(n=2)$ making it impossible to rule out the possibility that this is an artefact of data collection, precluding any definitive conclusions about an interactive effect between rehabilitation therapy and mobility on handicap. Both of the two subjects who were dependent in mobility received rehabilitation therapy, so no comparison to dependent individuals without therapy was possible. Furthermore, for 24 individuals it was unknown whether they received rehabilitation therapy, and so they were not included in this assessment. The addition of these unknown subjects could possibly change the observed relationships, although the majority ( $71 \%$ ) of these 24 subjects were independent in mobility at 3 months, with a similar mean RNL score to the rest of the independent subjects $(71.1 \pm 26.9)$ suggesting that these unknown 
individuals would not have made much difference to these results if their rehabilitation status had been known.

Therefore, although there is a suggestion of an interaction effect between rehabilitation therapy and motor ability, it is not possible to make any definitive conclusions about its effects due to small numbers, and the rehabilitation variable was therefore included in the model alone. (An interaction term combining rehabilitation therapy and psychological impairment was not significant.) It should be noted that the addition of the rehabilitation variable to the model with motor ability, depression and living arrangements slightly reduced the significance of the mobility variable, with a corresponding increase in the standard error of the estimate of its effect. This instability is likely due to the underlying interactions between level of mobility and the benefits of rehabilitation therapy, which cannot be appropriately controlled for in these analyses without a greater number of subjects in all subgroups.

In summary, motor ability, depression, living arrangements, and use of rehabilitation therapy appear to be associated with handicap at three months post-stroke, with some indication that rehabilitation therapy is most beneficial to those with some mobility dependence. According to the final statistical model with post-hoc comparisons, individuals who are more independent in mobility report much lower handicap (least-squares RNL mean of 70.4) than those who are moderately dependent (least-squares mean of 60.0) or dependent (least-squares mean of 41.9) in motor ability at three months post-stroke. Individuals who are depressed following their stroke report much greater handicap than those who are not (least squares RNL means of 49.0 vs 65.9 , respectively) when controlling for the effects of mobility, living arrangements and rehabilitation therapy. When comparing survivors of the same mobility and depression levels, individuals living at home at three months after their stroke onset report significantly less handicap than those in institutions (least squares means of 63.1 vs 51.8 ) when controlling for the effects of rehabilitation therapy. And finally, survivors receiving rehabilitation therapy reported greater handicap than those who did not, although therapy may be beneficial for those people who are more dependent in motor ability. Together, these four variables explained $42 \%$ of the variance in stroke handicap at three months post-stroke. 


\section{ii) At One Year Post Stroke}

One hundred and thirty-five subjects were assessed for handicap at one year after their stroke onset. Similar to the multivariate analyses performed for the three month data. the main independent variables, impairment and disability, were added in turn to an analysis of variance model to see if they explained the differences in handicap in these subjects. Again, the bivariate analyses indicated that handicap was greater in those individuals with more impairments and disabilities, but a multivariate analysis was performed to see how much each variable explained when controlling for the effects of the other factors.

Similar to the results at three months, level of stroke impairment at one year (Adams' Hemispheric Stroke Scale) was no longer statistically significant when motor ability was in the model, and depression at one year was again a statistically significant variable. However, in contrast to the three month data, we found that cognitive ability remained associated with handicap, even when motor ability and depression were in the model (Table 13). Together these three impairment and disability variables explained $37 \%$ of the variance in RNL scores at one year post-stroke.

Table 13

Multi-Way Analysis of Variance

Motor Ability, Cognitive Ability and Depression in Association with 1 Year RNL

$\mathrm{R}^{2}=0.373 \quad \mathrm{~F}_{\mathrm{S} .125}=14.87 \mathrm{p}<0.0001$

$\begin{array}{llll}\text { Variable } & \text { df } & \text { EValue } & \mathrm{Pr}>\mathrm{F}\end{array}$

$\begin{array}{llll}\text { Motor Ability } & 2 & 12.69 & 0.0001\end{array}$

$\begin{array}{llll}\text { Cognitive Ability } & 2 & 9.01 & 0.0002\end{array}$

$\begin{array}{llll}\text { Depression } & 1 & 7.76 & 0.0062\end{array}$

An examination of the least-squares adjusted means indicates that individuals who were more independent in motor ability had higher RNL scores (average of 64.1 ) than those who were moderately dependent (mean RNL of 48.2) or dependent (mean RNL of 34.0 ) in mobility, after adjusting for the effects of the other two variables in the model. Similarly, cognitively independent individuals had higher RNL scores (average of 67.2) compared to those who were dependent or moderately dependent in cognition (mean RNL of 23.8 and 55.4, respectively). 
Finally, survivors who experienced a dysphoric mood after their stroke reported significantly lower RNL scores than those who were not depressed (least-squares means of 44.5 vs 53.0 , respectively).

So once again, psychological impairment and both physical and cognitive disability appear to be important determinants of handicap in the long term stages of stroke recovery, suggesting some validity to the ICIDH model. However, in order to determine the impact of other variables on these relationships, particularly the interactive effects of environmental factors, the other potentially important variables were added to this basic model in turn to see if they added anything to the model, or to see whether they changed any of the alrcady observed relationships.

In these series of analyses, age, comorbid conditions, intercurrent illnesses (both stroke related and non-stroke related), pre-stroke disability, side or type of stroke, level of education, pre-stroke quality of life, living arrangements and use of rehabilitation therapy were not important in predicting handicap at one year post stroke over and above the effects of motor ability, cognitive ability and depression. The addition of age to the model as either a categorical or continuous variable with the established impairment and disability variables already present did not change the effects of these significant variables, nor did it raise the standard error of their estimates. Therefore, age is not a confounder and is not the underlying reason for the observed relationships between impairment, disability and handicap. Similarly, pre-stroke disability, stroke history and comorbid conditions were not confounding variables; they did not alter the effects of the predictor variables on handicap nor did they substantially raise the standard errors of their estimates.

However, although stroke history did not have any confounding effects on handicap, stroke history was independently associated with handicap over and above motor and cognitive disability and depression (Table 14). Individuals who had suffered a previous stroke reported significantly more handicap than those who had not (least-squares adjusted RNL mean of 39.8 vs 47.6 , respectively). This suggests that there is something about a previous stroke which is important to long term handicap that is not picked up by the measure of disability or impairment. Because the Adams' Stroke Scale is a unilateral impairment measure, neurological deficits due to previous lesions occurring in the contralateral hemisphere to the current event would not be 
documented. It is, therefore, likely that residual impairment from a previous stroke is the reason why this variable is important over and above the current impairment and disability levels. These previous impairments may only be important at one year and not at three months because these deficits may only become salient in the longer term when the sequelae from the current deficits have subsided.

Table 14

Multi-Way Analysis of Variance

Motor Ability, Cognitive Ability, Depression, Stroke History, Sex and Marital Status in Association with 1 Year RNL

\begin{tabular}{llll}
\hline$R^{2}=0.438$ & $F=10.49$ & p<0.0001 \\
Variable & df & E Value & \\
Motor Ability & 2 & 12.86 & 0.0001 \\
Cognitive Ability & 2 & 12.02 & 0.0001 \\
Depression & 1 & 6.79 & 0.0103 \\
Stroke History & 1 & 6.55 & 0.0117 \\
Marital Status & 1 & 2.69 & 0.1037 \\
Sex & 1 & 0.35 & 0.5559 \\
Marital Status x Sex & 1 & 4.42 & 0.0377 \\
\hline
\end{tabular}

Interestingly, the dichotomous married variable was also associated with handicap at one year, although it was important in interaction with the respondent's $\operatorname{sex}(p<0.04)$. (The more conservative practice of retaining the individual variables of the interaction term on their own was followed.) When looking at the breakdown of mean RNL scores by sex and marital status (Table 15), one can see that males who were married reported higher RNL scores than married fernales.

Table 15

Interaction Effect of Marital Status and Sex Average RNL ( \pm s.d.) Scores by Marital Status and Sex

\begin{tabular}{lcc}
\hline & Sex & Female \\
Marital Status & & \\
Married & $83.2 \pm 16.0$ & $74.0 \pm 23.9$ \\
& $(\mathrm{n}=53)$ & $(\mathrm{n}=22)$ \\
Not Married & $78.6 \pm 18.9$ & $79.4 \odot 15.7$ \\
& $(\mathrm{n}=18)$ & $(\mathrm{n}=42)$ \\
\hline
\end{tabular}


Additionally, males who were married reported higher scores than unmarried males. while unmarried females reported higher scores than married females. Therefore, it would appear that marriage is likely to reduce handicap in male stroke survivors, but marriage is not similarly beneficial for women. (Interaction terms combining marital status with the disability and impairment variables were not statistically significant.)

With these other factors in the model, we again examined the least-squares means of the impairment and disability variables in post-hoc comparisons to identify the source of differences in handicap scores for each main variable, after adjusting for the effects of the other variables in the model. We found that individuals who were more independent in motor ability reported higher RNL scores (58.3) than those who were dependent or moderately dependent ( 30.2 and 42.6, respectively). Similarly, individuals who were more independent in cognition at one year reported significantly less handicap (mean RNL of 64.5) than those who were moderately dependent or dependent (51.2 and 15.4 respectively). Depressed individuals at one year reported significantly greater handicap than those who were not depressed (mean RNL scores of 39.8 vs 47.6 , respectively). Together all six variables explained $44 \%$ of the variance in handicap in those who survived to one year post-stroke.

Although age was not a confounder, there was some suggestion that it may play an important role in handicap creation over and above the effects of impairment and disability. In a separate model (not shown), age was found to be significantly associated with handicap when added to a model with only motor ability, cognitive ability and depression $\left(F_{2.123}=3.33, p=0.04\right)$. There has been some discussion in the recent medical literature about the importance of age as an independent risk factor for poor outcome (Manolio \& Furberg, 1992; Chelluri, Pinsky, Donahoe, \& Grenvik, 1993), prompting a controversial debate about using age as a criterion for health care rationing (Kilner, 1989; Jecker, 1991). The significance of age as an independent predictor of handicap may have been picking up on this finding. However, it was not significant when added to the model which included stroke history, marital status and sex. The reasons for this change may be due to the underlying demographic profile of subjects by marital status and sex. We saw from the interaction term in the final multivariate model that marital status is beneficial for males but not for females. We also know that more of our male subjects were married (71\%) than our 
female subjects (29\%) and that women tended to be older ( $>75$ years) than men. Therefore. because of the demographic profile of stroke, more of the female stroke survivors in the study were older and not married while male survivors were more likely to be younger and married. So when age was an important effect on its own, it may just have been because of the underlying differences between marital status and sex by age group, and when controlling for these two variables, age no longer contributed to the explanation of handicap on its own. However, there may be insufficient power in this study to detect any independent effects of age over and above marital status and sex, and further research needs to be conducted with a greater number of study subjects to tease out its relative importance. 


\section{DISCUSSION}

The purpose of this research project was to examine the extent to which the impairments and disabilities associated with a stroke precipitate handicap in stroke survivors. It was hypothesized that individuals with more extensive impairments and disabilities would experience greater handicap following their stroke. Have our results supported this hypothesis? Each follow-up period will be discussed in turn.

\section{Handicap at Three Months Post Stroke}

We found that motor disability and psychological impairment were associated with greater handicap in stroke survivors at three months after stroke onset. Individuals who had more motor disability reported more handicap than those who were more independent, and individuals experiencing post-stroke depression were more likely to report greater handicap. Although we found that stroke impairment was associated with handicap in the bivariate analyses, it was no longer significant when coupled with motor ability in the multivariate analyses. Similarly, cognitive disability was not associated with handicap over and above the effects of psychological impairment. When depression was added to the model with the motor and cognitive disability variables already present, cognitive disability was no longer significant. This suggests that cognitive disability following stroke and mood state are highly correlated, but that depression explains more of the variance in handicap at three months than cognitive disability. In fact, depression may have been the underlying reason why cognitive disability was associated with handicap in the bivariate analyses since individuals who are depressed may be more likely to experience problems in communication and problem solving (Robinson, Bolla-Wilson, Kaplan, Lipsey, \& Price, 1986), as measured by the cognitive dimension of the FIM.

Together motor disability and depression explained $41 \%$ of the variance in handicap at three months. Therefore, impairment and disability are associated with handicap in stroke survivors, explaining almost half the variability in handicap scores in the subacute phase of recovery. This is a high multiple correlation coefficient $\left(R^{2}\right)$, rarely observed in observational studies, lending considerable support to the theoretical framework of the ICIDH model which suggests that impairments and disabilities create handicap. However, other variables and environmental 
factors were also hypothesized to be important to this relationship, and their effects were also explored.

First, age, comorbidity, pre-stroke disability and a history of a previous stroke were thought to be potential confounders. Older individuals or those who experienced other illnesses or disabilities prior to their stroke onset were thought to be more likely to report greater disability and handicap after their stroke, regardless of the impact of the stroke. However, this was not supported by the results. The observed association between impairments, disabilities and handicap could not be explained away by the underlying influence of age, comorbid conditions. stroke history or pre-stroke level of impairment or disability, nor did these factors have independent effects on handicap.

Environmental factors were specifically postulated to be important influences in the handicap creation process. As outlined by the CSICIDH (Canadian Society for the ICIDH, 1991), impairments and disabilities may only become handicaps in interaction with the surrounding environment, and external factors may also moderate the extent to which impairments and disabilities create handicaps. Although it was not possible to collect information on all potentially important environmental factors in this study, a number of external factors were indeed found to influence handicap.

First, social resources were found to be beneficial for post-stroke handicap. Individuals who lived at home at three months reported less handicap than those in institutions, when controlling for the effects of impairment and disability. This means that across individuals with the same level of motor disability and psychological impairment, residence at home was more beneficial than residence in an institution. One would have thought that institutional residence is associated with greater handicap because individuals requiring medical care are more likely to have more disabilities and impairments. However, this finding was observed when controlling for the underlying level of illness. Therefore, something about living at home is beneficial to the social well-being of stroke survivors at three months which is relevant to individuals at all levels of impairment or disability. One might assume that these benefits originate from the presence of a companion or spouse who might be in closer proximity at home. However, the same benefits were not observed for people living with someone else, nor were they observed for married 
compared to non-married individuals. Therefore, this phenomenon is picking up on something entirely separate from companionship. What is it about home residence that is beneficial to handicap? Perhaps it is an issue of control. For those individuals living at home, there may be a greater sense of control than in institutional environments, which tand to be highly structured with designated waking, eating and bathing times. Individuals in institutional care are less likely to have control over their activities, which may be a source of frustration, impeding satisfaction with life habits. In fact, it may be inappropriate to consider handicap for those residing in institutions since social life habits are not easily demonstrable.

Our results also suggest that the use of health services such as rehabilitation therapy may reduce handicap following stroke. We found that the benefits of rehabilitation therapy appeared to vary depending on the individual's level of mobility dependence suggesting that rehabilitation may alleviate handicap in those with more dependence. If an individual was moderately dependent in mobility, rehabilitation therapy appeared to minimize the impact of this disability on handicap, whereas for individuals with less disability, such benefits were not observed. This is an important finding considering that some studies have demonstrated no benefits from rehabilitation over and above the natural pattern of recovery (Dobkin, 1989). However, as articulated by Evans (Evans, Connis, Bishop, Hendricks, \& Haselkorn, 1994), few of the rehabilitation studies have examined outcome beyond a measure of functional activity. The benefits of rehabilitation therapy may exist when considering the broader perspective of individuals, such as handicap, as found in this study. However, the number of subjects in the more dependent groups was small, so it is only possible to speculate about the extent of these interaction effects. Further research needs to be done to explore the effects of rehabilitation therapy after stroke, with attention paid to such factors as type of therapy, length of time therapy is received and discharge destination.

Although these two environmental factors were statistically significant, they only raised the $\mathrm{R}^{2}$ of the whole predictive model to $42 \%$ (one additional percentage point), suggesting that although they may be clinically and intuitively important, most of the variability in handicap in the immediate phase after a stroke is explained by impairment and disability. 
No other variables were found to affect handicap when controlling for the effects of motor disability and depression. Side or type of stroke, sex, race, and education did not help to predict handicap in those individuals who survived to three months. Although it was hypothesized that SES might be an important resource moderating the effect of impairment and disability on handicap, this finding did not materialize in the results. This is similar to the results from other studies which have found no effect of socioeconomic group on quality of life (Ahlsio, Britton, Murray, \& Theorell, 1984). This is not to say that wealth is not influential on handicap, but perhaps there was insufficient variability in SES in this group to detect an effect since they were mostly from a higher income population, or perhaps level of education attained is an inadequate proxy for SES.

Furthermore, although stroke-related illnesses occurring between onset and three months were associated with greater handicap in the bivariate analyses, this finding did not materialize in the multivariate tests. Perhaps the reason for the importance of intercurrent illness in the bivariate tests was due to the underlying association between intercurrent illness and stroke related impairment and disability. In fact, these stroke related illnesses were either seizures, falls, dementia or depression. So the reason why intercurrent illnesses were important in the bivariate analyses was likely due to the underlying importance of post-stroke depression, which after being accounted for on its own, negated the importance of the other illness variable. Similarly, although pre-stroke quality of life appeared to be linearly related to handicap in the bivariate results, this finding was not observed in the multivariate analyses. Perhaps the effects of pre-stroke quality of life were simply not strong enough to contribute to the model over and above the impairment, disability and environmental variable.

\section{Handicap at One Year Post Stroke}

Similar to the three month analysis, we found that motor disability and psychological impairment were also associated with handicap in the more long term stages of stroke recovery. Again, stroke severity was not associated with handicap over and above the effects of motor disability. However, in contrast to the three month data, cognitive disability remained associated with handicap over and above the effects of psychological impairment. Therefore, cognitive 
disability is an important limitation for long term stroke survivors in addition to psychological impairment. Other studies have found that individuals with post-stroke depression have significantly less recovery from cognitive disability after six months of follow-up (Robinson. Bolla-Wilson, Kaplan, Lipsey, \& Price, 1986). The importance of cognitive disability at one year may reflect this lingering impact of depression. Together these three impairment and disability variables explained $37 \%$ of the variance in handicap at one year post stroke. Although this is slightly less than the proportion explained by the impairment and disability variables at three months, it is nevertheless, quite high, lending further support to the theoretical framework of the ICIDH model.

The possible confounding effects of other variables on these relationships was also explored in the one year data. Although age, comorbidity and pre-stroke disability were thought to be possible confounders because of their potential relationship with both disability and handicap, they were subsequently not found to have an effect on the observed relationships. However, impairments from a previous stroke were found to have an independent predictive effect on handicap over and above disability and psychological impairment.

In terms of the importance of environmental factors at one year, the use of rehabilitation therapy after three months was not influential over and above impairment and disability. This is in contrast to the findings at three months, suggesting that the benefits of rehabilitation therapy may only occur in the more immediate period following stroke. Rehabilitation therapy may only be advantageous in the early stages (less than three months) at the time when spontaneous neurological recovery is occurring, since little benefit has been demonstrated elsewhere after three months (Smith, Goldenberg, \& Ashburn, 1981). Or, rehabilitation therapy may not be statistically significant at one year either because individuals have already benefitted from it and have had a reduction in handicap, or have made no progress with it and remained handicapped. It is possible that these two effects may have cancelled each other out in order to generate a null finding.

However, although health services were not found to be influential, social resources did appear to play a moderating role on handicap in survivors at one year. Specifically, marriage appeared to reduce handicap in male survivors after controlling for the effects of impairment and 
disability. Males with a spouse reported less handicap than males who did not have a spouse. But, surprisingly, this finding was not observed in female stroke survivors. In fact, married women reported more handicap than unmarried women. There is an extensive literature on the psycho-sociological effects of marriage. A number of sociological studies have found that marriage is beneficial to the health and well-being of individuals (Weingarten. 1985: Gove, Hughes, \& Style, 1983; Kessler \& Essex, 1982) perhaps because the presence of a spouse acts as a social support which buffers the impact of stressful life events (Thoits, 1982) or facilitates healthy behaviour such as eating well or seeking prompt medical attention to any symptoms (Muhlenkamp \& Sayles, 1986). Marriage has also been shown to be beneficial to stroke survivors because it facilitates a discharge home rather than extended stay in hospital (Smurawska, Alexandrov, Bladin, \& Norris, 1994). Men in particular may benefit from marriage because they tend to rely almost exclusively on their spouse for a source of support, with few alternative sources of support (Stroebe \& Stroebe, 1983).

However, while this beneficial effect of marriage may have been active for males in this study, a similar effect was not observed for females. In fact, marriage appeared to be detrimental to the well-being of women following stroke. There is conflicting evidence about the effects of marriage for women in the scientific literature. For instance, research has demonstrated that married women have significantly lower levels of depression than single women (Kandel, Davies, \& Raveis, 1985), while other studies have demonstrated that marriage is not beneficial for women's health and well-being (Gove \& Tudor, 1973). The detrimental impact of marriage for women may lie in the fact that multiple roles in a married woman's life can be stressful, leading to "role overload" (Kandel, Davies, \& Raveis, 1985). Women tend to have more social role responsibilities than men, and they assume a disproportionate share of the responsibility of these roles than men (Cleary \& Mechanic, 1983). The demands of these multiple roles coupled with the unequal sharing of these roles can be a source of stress and burden for women (Cleary \& Mechanic, 1983; Gove, 1978). However, some studies have found that these multiple roles prove beneficial to the health of women (Verbrugge, 1983; Thoits, 1983) in part because of the benefits of each role in terms of social involvement and personal reward. On the other hand, instrumental roles associated with family, such as taking care of the home, are associated with 
high levels of stress and are the least satisfactory of the social roles in which women are involved (Kandel, Davies, \& Raveis, 1985).

There is, therefore, obvious disagreement about the effects of marital status on health amongst gender. The results of this study seem to reinforce the finding that marital status is beneficial, but only for men. The less favourable effects of marriage for women may exist because the effects of a stroke amplify the stress of domestic instrumental roles which may cancel out any of the potential benefits from marriage. Since women (and particulariy those in this cohort) may take on most of the domestic responsibilities in a married relationship, female stroke survivors who are married may be burdened by chores or responsibilities in providing domestic tasks for a husband, or feelings of inadequacy if these tasks cannot be performed. Men, on the other hand, may benefit from the traditional domestic relationships following stroke since their wives may provide the support in daily activities that serves to enhance their well-being. These different role responsibilities have been suggested elsewhere as a possible explanation for differential recovery patterns in men and women in a randomized trial of team care versus traditional hospital care following stroke (Wood-Dauphinee et al., 1984).

It is interesting that similar beneficial effects to those of marriage were not observed when comparing handicap scores in those who lived alone to those who lived with either family or friends. Therefore, there must be something in the nature of married life itself which is beneficial to males, over and above the presence of someone else in the home. In the social support literature, support from intimates has been identified as one of the key components of effective supportive relationships (Thoits, 1985). This intimacy may be what is active here for males in the form of a spousal relationship over and above straight companionship. For instance, male survivors in a health care facility may benefit from the intimacy and a shared history with a spouse even if they are not actually living with their wives at home. And similarly, there must be something in the marital relationship which is burdensome to female stroke survivors, over and above the presence of additional people. As discussed, this may be rooted in a sense of failed expectations of roles.

In terms of the effects of other variables on the association between impairment, disability and environmental factors on post-stroke handicap, a history of a previous stroke also became 
important in one year survivors. Together, a history of a previous stroke and maritai status in interaction with sex explained an additional $7 \%$ of the variability in handicap over and above the main effects of disability and impairment, bringing the total $\mathbf{R}^{2}$ of the model with all six variables to $44 \%$. Again, most of the variability in one year handicap was due to residual impairments and disabilities, but environmental factors also appeared to have an impact, more so than at three months.

No other variables were additionally important in explaining one year handicap. Although residence at home was beneficial over an institution in the bivariate analyses, this was not important in the multivariate model at one year, perhaps due to its underlying correlation with either disability level or marital status. The importance of living arrangements at three months but not at one year may be because most individuals who had been residing in an institution would have been discharged by one year, or if they had not been discharged, they would be too ill such that other impairment and disability factors would override the importance of residence as a predictive factor. Again, pre-stroke quality of life was not important in the multivariate model, although there had been some suggestion of its importance in the bivariate results. again most likely due to its lesser importance in comparison to other variables in the model.

\section{Summary}

In summary, this research project has found that impairments and disabilities following a stroke do significantly impact on the life habits of survivors. Specifically, motor disability and post-stroke depression were found to be repeatedly associated with handicap throughout the recovery period. These results support those of other studies in this area which have also found that motor disability is a significant burden for stroke survivors (Dejong \& Branch, 1982; Henley, Pettit, Todd-Pokropek, \& Tupper, 1985), as well as for elderly people in general (Campbell et al., 1994; Kempen \& Suurmeijer, 1991; Verbrugge, Lepkowski, \& Imanaka, 1989). In fact, motor disability is perceived as sufficiently dire that in a study assessing patient preferences for outcome following stroke, individuals expressed a preference for death over severe motor disability (Solomon, Glick, Russo, Lee, \& Schulman, 1994). 
The importance of depression in stroke survivors has also not gone unnoticed. Stroke survivors with clinically diagnosed depression have been found to have difficulty returning to their usual social activities, even up to two years after onset (Parikh et al., 1990). Therefore, the importance of recognizing and appropriately treating post-stroke depression cannot be overemphasized, particularly since it responds so well to pharmacologic treatment (Lipsey, Robinson, \& Pearlson, 1984; Reding, Orto, \& Winter, 1986). The propensity for elderly individuals to reject diagnoses of depression and for health practitioners to fail to recognize depressive symptoms (Goleman, 1995) is a bad combination which puts stroke survivors at unnecessary risk for compromised social and life satisfaction. Furthermore, the effects of depression are not limited to handicap alone; stroke survivors who are depressed show a much slower recovery from physical impairments (Starkstein, Parikh, \& Robinson, 1987), perhaps because they are less able to adhere to rehabilitation (Sinyor et al., 1986) or medication regimes (Goleman, 1995) which may further elevate their risk for handicap, not to mention their risk for a subsequent stroke (Simonsick, Wallace, Blazer, \& Berkman, 1995).

Cognitive disability and residual impairment from a previous stroke also contribute to poststroke handicap, but only in the longer term when the more immediate effects of the stroke have subsided and survivors may come to notice more subtle damages. The importance of cognitive ability has also been found to be important for functional outcome after stroke in other studies (Galski, Bruno, Zorowitz, \& Walker, 1993; Robinson, Bolla-Wilson, Kaplan, Lipsey, \& Price, 1986). Although environmental factors, such as place of residence and the use of rehabilitation services, are statistically significant at three months, they contribute minimally to the overall model. Environmental factors become much more important in the long term stages of recovery. With the addition of environmental factors, the one year model explains almost half of the variance in handicap. Specifically, a spouse is advantageous to male survivors at this stage, indicating that family resources need to be reinforced so that these benefits can be sustained. 


\section{Validity of the ICIDH Model}

The results of this empirical analysis provide insight into the validity of the conceptual relationships underlying the ICIDH model from the perspective of the stroke experience. In the first place, the results lend support to the basic model of the ICIDH (World Health Organization, 1980) which postulates a sequenced linear relationship in which impairments lead to disabilities which then lead to handicaps (Figure 1). The fact that neurological impairment, as measured by the Adams' Hemispheric Stroke Scale, was no longer a statistically significant variable when motor disability was included in the statistical model, suggests that there may indeed by some sequencing of impairment and disability in the creation of handicap following stroke. Furthermore, the fact that cognitive disability at one year is likely a direct consequence of depression (Robinson, Bolla-Wilson, Kaplan, Lipsey, \& Price, 1986), also suggests a possible linear sequencing between psychological impairment, cognitive disability and handicap. However, we also found that psychological impairment alone affects handicap in stroke survivors, without the intervening presence of disability. Therefore, there is evidence for the validity of a model which allows for the direct effects of impairment on handicap, without the intervening presence of disability. This was evident at both the three month and one year followup periods.

Furthermore, this research also found evidence in support of environmental models such as the one suggested by the Canadian Society for the ICIDH which maintains that handicap is a situational result of impairments and disabilities in interaction with the person's surrounding environment (Figure 2). Although there was insufficient data to fully explore an interaction term between the use of rehabilitation therapy at three months and motor disability, there was an indication that the effects of disability on handicap varied according to the use of rehabilitation services. Figure 4 illustrates the suggested interaction effect that was found at the three month follow-up period. Although the small number of subjects in the mobility dependent group precludes their inclusion in the graph and therefore any definitive conclusions, the divergent lines suggest that although handicap increases as motor disability increases, the rate of increase is less in those who received rehabilitation therapy. Therefore, the effects of disability on handicap seem to vary according to the use of rehabilitation therapy. The facilitative effect of 
this environmental resource may reduce the impact of moderate motor disability on the life habits of stroke survivors. This has implications for the distribution of health care resources, and further research needs to be done with a greater spectrum of subjects in order to further clarify this relationship.

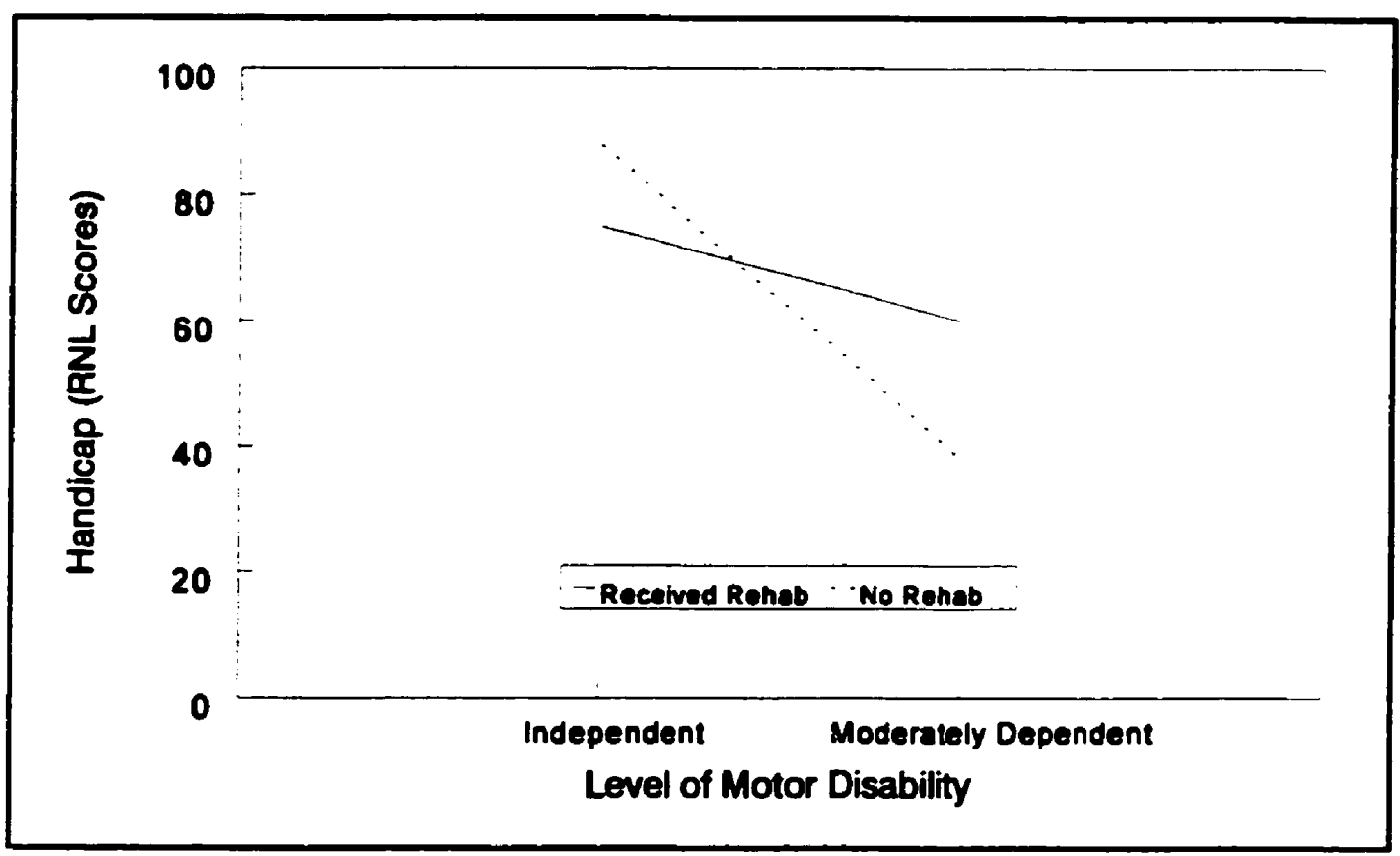

Figure 4 Effect of disability on handicap according to the use of rehabilitation therapy at three months

Additionally, the emphasis on interaction in the CSICIDH model serves to highlight the conceptual and empirical importance of other interaction terms not specifically articulated in the model. For instance, at the one year follow-up period, there was evidence of an interaction effect between marital status and sex. Although this interaction term does not incorporate disability or impairment, as outlined in the CSICIDH model, it does provide an understanding of the possible mechanisms through which environmental factors themselves may interact to affect handicap. As depicted in Figure 5, the divergent lines indicate that the effects of marital status on handicap vary by the sex of the subject. The presence of a spouse seems to reduce handicap for male stroke survivors, but increase handicap for female survivors. Although there was no interaction between marital status and impairment or disability, this interaction term nevertheless 
serves to emphasize the explicit empirical examination of interaction effects advocated in the theoretical model.

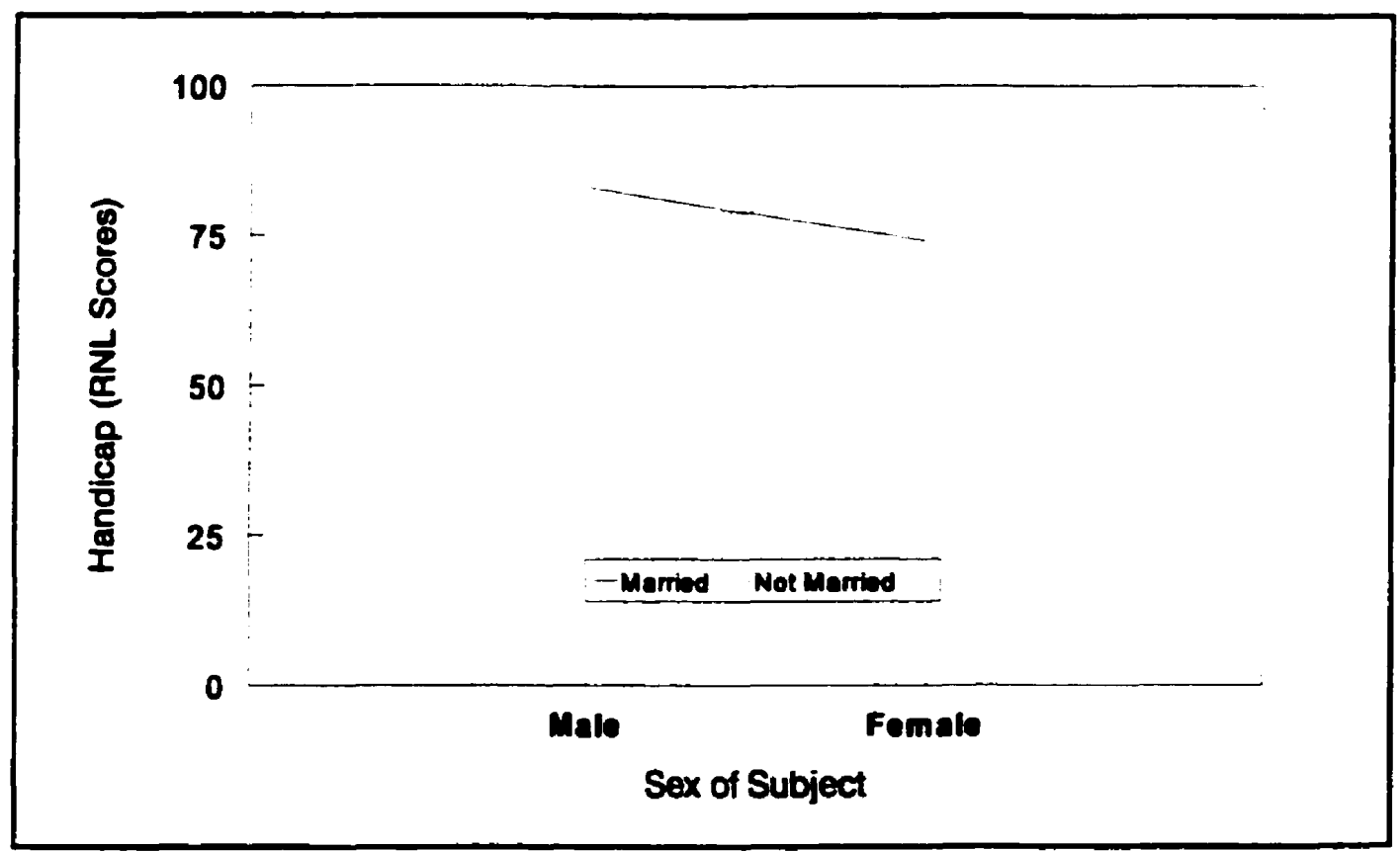

Figure 5 Effect of marital status on handicap according to the sex of the subject at one year

However, while these results suggest that there may be some validity to models such as the one from the CSICIDH (Figure 2), which depicts the handicaps creation process as an interaction between impairments or disabilities and environmental factors, this study also found that impairment and disability can affect handicap independently, without any interaction with the surrounding environment. Specifically, motor disability, cognitive disability and depression were found to have a direct impact on handicap without any statistically significant interaction with environmental variables. Of course, this may be because this study did not collect enough data on potential environmental factors or that there was insufficient statistical power to detect any interaction effects. Environmental factors are often complex, acting in ways that are not easily measurable. Motor disability, in particular, may, in fact, have been associated with handicap only because of the underlying importance of the environment. Stroke survivors with difficulties in mobility may experience handicap situations because their environment is not receptive to their needs. Structural factors or unmodified housing may have been the source of 
the social restriction resulting from this motor disability, and if measured, may have revealed a statistically significant interaction term with motor disability and these environmental factors.

However, for depression and cognitive disability, it is difficult to discern which environmental factors may have been responsible for their importance in the creation of handicap situations. Social barriers certainly may be important in the appropriate treatment of psychological impairment. The stigma surrounding mental illness, particularly in this cohort, may prevent stroke survivors from seeking treatment for depression, and the medical profession may also be inadequately trained to recognize depressive symptoms in the elderly (Goleman, 1995). But, while these social environmental factors may be working to sustain the association of depression with handicap throughout the one year recovery period, they are not responsible for its initial importance to the stroke survivor. Depression may affect handicap simply because it is a difficult and burdensome impairment in and of itself. It is difficult to believe that the removal of any social or physical barriers would eliminate the effects of post-stroke depression on handicap. Therefore, the results of this thesis indicate that handicap can originate as a result of impairments and disabilities on their own, not necessarily due to any interaction with the surrounding environment. This is not recognized by the Canadian model (Canadian Society for the ICIDH, 1991).

Furthermore, the results of this thesis also indicate that environmental factors may have an independent, non-interactive effect on handicap, which is also not depicted in the Canadian model, although perhaps it is implicit to the developers. Living arrangements and marital status seem to affect handicap independently, without interacting with disability or impairment. Residence at home was found to be a facilitative environmental resource following stroke, reducing handicap independently of impairment and disability. Similarly, marriage was found to be a positive resource for males, but acted as an obstacle for females. Again, these independent effects may simply be a result of insufficient statistical power to detect an interaction effect with disability or impairment. But it is also possible that environmental factors, such as living arrangements and marriage, have a direct impact on handicap across all levels of disability or impairment. 
It is difficult to decide whether these independent environmental effects should be included in the theoretical model since they exist outside the illness experience and are, therefore. typically not included in the ICIDH models. However, it is also possible that these resources take on a different meaning following illness, over and above the general life experience. This may involve an indirect, as opposed to a direct, effect, with impairments and disabilities acting to mobilize resources or obstacles following stroke, rather than interacting with them. The only way to disentangle the effects of the stroke experience from the overall life experience is to undertake a similar study with a matched control group to see if these environmental factors have a different impact within the illness experience. It may then be necessary to develop a model which includes the indirect effects of environmental factors on handicap within the health experience.

Overall, it appears that neither model depicted in Figure 1 or Figure 2 adequately captures the genesis of handicap following stroke. As recognized by others, the basic model of the ICIDH in Figure 1 neglects to incorporate the role of environmental factors and fails to allow for the direct effects of impairments on handicap without the intervening presence of disability. On the other hand, while other models, like the one in Figure 2, incorporate environmental factors, they emphasize the interaction between impairments, disabilities and environmental factors to the exclusion of any independent effects. In fact, none of the models developed to date seems to adequately capture the relationships uncovered in this study. Nevertheless, it is unlikely that one single model will ever capture all the possible dynamics of a particular illness or trauma experience. The ICIDH is a broad tool which, in spite of its limitations, encourages and facilitates an investigation and deeper understanding of the illness experience. In this regard, it has been a useful tool with which to approach the stroke experience.

But apart from revealing some of the conceptual relationships underlying the ICIDH models, this research has also provided information on the nature of environmental factors acting within the stroke experience. First, although most of the external factors had a positive, facilitative effect on reducing handicap, marriage was perceived as an "obstacle" to female survivors, as depicted by the CSICIDH model. In terms of the terminology used by Verbrugge and Jette (Verbrugge \& Jette, 1993) the environmental factors identified in this research are extra- 
individual in nature, existing outside the individual, such as place of residence, use of rehabilitation therapy and marital status. However, some of the underlying reasons for the importance of these environmental factors may be intra-individual, that is, intrinsic to the individual. For instance, the importance of home as opposed to institutional residence at three months may be due to intra-individual factors such as feelings of personal cont- -1 over the surrounding environment.

Finally, the environmental factors identified in this research were both pre-existing and responsive, as defined by Badley (1995). Rehabilitation therapy is a responsive factor that is only marshalled in response to a resulting disability. Whereas, pre-existing factors, such as marital status, were present in the individual's surrounding environment before the stroke, but may take on a different meaning (become responsive) in the face of a stroke. Similarly, living arrangements can also be viewed as both pre-existing and responsive. Residence in a health facility is a resource that is activated in response to the stroke, whereas home residence is a resource that was present before the event, although, it may also take on a different meaning or value following illness, as discussed earlier.

Overall, this research has yielded further information about the nature of environmental factors in the handicaps creation process, providing insight into their character and their interactions with underlying disability. It has also provided an empirical evaluation of the validity of the conceptual framework of the ICIDH lending considerable support to the importance of both disability and impairment in the handicaps creation process, and emphasizing the importance of environmental factors with some evidence of an interaction with disability.

\section{Limitations}

\section{i) Study Design}

Although the data from this study were collected in a prospective cohort format, they were analyzed cross-sectionally. This was done to complement the current body of research in this area which is descriptive in nature. However, unlike typical cross-sectional designs, the knowledge of subjects lost to follow-up prevents any over-generalizations of the study results. Nevertheless, it must be kept in mind that this study does not provide information about changes 
in handicap over time, nor on the effects of changing impairments and disabilities on handicap. Such analyses are reserved for future studies.

\section{ii) Potential Biases}

One of the well-known limitations of prospective cohort designs, is the trouble that can arise due to losses to follow-up throughout the study period (Hennekens \& Buring, 1987). And indeed, the potential limitations of this particular research project are rooted in the losses to follow-up that occurred. As outlined by Elwood (Elwood, 1988), losses to follow-up can have three effects on study results: they can modify the hypothesis under investigation, affect the external validity of the study, or affect the internal validity.

In the first place, the hypothesis under investigation in this study was indeed modified by the characteristics of the non-participants. The objective of this thesis was to investigate the impact of impairments and disabilities on handicap in stroke survivors. But, as outlined earlier, it was not possible to assess handicap in those who were too ill to contemplate the impact of their stroke on their social roles and activities. Therefore, the original hypothesis of the study was modified so that it pertained only to those stroke survivors who were well enough to report on their handicap situation.

These selection criteria also affect the external validity of the study. External validity is the extent to which the study results can be applied to the wider population of interest. Although it was originally intended to better understand the determinants of handicap in all stroke survivors, the subset of individuals who participated in this study restrict the population to which the results can be applied. In the first place, stroke patients admitted to Sunnybrook Hospital tend to be older, with a higher education and income than the rest of the Metropolitan Toronto population. Second, only hemispheric strokes were included in this study, preventing any conclusions about handicap following a vertebrobasilar event. Third, because all subjects in this study received extra attention throughout the one year follow-up period as part of the study protocol, the results may not be generalizable to stroke patients who do not receive similar follow-up. Finally, due to the nature of handicap, only hemispheric stroke survivors who were well enough to report for themselves were included in the handicap analysis. By examining 
handicap in these subjects, the study findings can only be applied to hemispheric stroke survivors with similar characteristics.

However, there were subjects who fell within these inclusion criteria who did not complete a handicap assessment. Although there were 248 survivors who were well enough to be assessed for handicap, only $164(66.1 \%)$ actually participated in the assessment, and the participants were found to differ in some respects from the non-participants. Therefore, although all hemispheric stroke survivors who were well enough to report for themselves were eligible for the handicap component of this study, the slightly different characteristics of the individuals who actually participated may further restrict the population to which these study findings can be generalized. Because participants tended to be younger with a better pre-stroke quality of life than nonparticipants, the observed relationships between impairment, disability and handicap may exist to a different degree in all hemispheric stroke survivors well enough to report for themselves. Furthermore, these results may be more appropriate to hemispheric stroke survivors who have a similar proportion of hemorrhages and diabetic history to the participants. Therefore, although the differences between the participants and the group of interest are not substantial, any extension of the results of this study to all hemispheric stroke survivors well enough to report for themselves should be made with these characteristics in mind.

But perhaps a greater problem is that these differences between participants and nonparticipants may compromise the internal validity of the study findings. External validity is of little value if the internal validity is suspect. Internal validity is the extent to which the difference in handicap scores observed between individuals in this study is really due to the effects of impairment and disability. The danger here lies in the fact that the results of this study may not reflect the impact of impairments and disability on handicap, but may simply be an isolated finding due to the specific characteristics of the participants. It is important to consider whether the observed relationship between impairment, disability and handicap would have been different if the non-participants had been included in the analysis.

As articulated by Hennekens (Hennekens \& Buring, 1987), a biased estimate of the relationship between exposure and outcome will only result if non-response is related to both the exposure and other risk factors for the outcome under study. For instance, if the non-participants 
were more likely to be disabled than the participants, and, independent of these disabilities were also at greater risk for handicap, then a biased estimate of the relationship between disability and handicap would result. Although a greater proportion of the non-participants in this study had a hemispheric infarct, no differences have been found between hemorrhages and infarcts with respect to handicap (de Haan, Limburg, Van der Meulen, Jacobs, \& Aaronson, 1995). Although non-participants reported a worse pre-stroke quality of life than the participants, this would only have a possible effect on handicap, but not the likelihood of disability. Therefore, while these non-participants may lead to a possible over or underestimation of handicap, they are unlikely to have changed the estimate of association of handicap with disability.

However, age and diabetes history could create potential biases because they could be related to both exposure and outcome. Individuals who are older or who have a history of diabetes may be more likely to have impairments and disabilities. Furthermore, individuals who are older or who live with diabetes may be more likely to report greater handicap apart from any resulting disability. Therefore, the exclusion of these non-participants may underestimate handicap in the disabled, and may also generate an artificially low estimate of the difference in handicap between disabled and non-disabled.

However, the participants and non-participants were more similar than they were different, and it is questionable whether the differences that did exist could have had a substantial effect on the observed association between impairment, disability and handicap. Furthermore, even if these differences were causing a systematic error in the observed association, they would more than likely only have reduced the estimate of the effect. Since the non-participants may have been more likely to have disabilities and handicap, the observed estimate of association would only have been greater if these non-participants were included. Therefore, the effects of losses to follow-up, if any, would only have served to underestimate the true association, and would not have nullified the findings in this analysis.

Apart from losses to follow-up, biases may also result due to other aspects of the study design. Since study subjects were reporting on their handicap when aware of their exposure, individuals with disabilities may overestimate their level of handicap compared to those without disabilities or impairments. However, since handicap is a subjective construct, there is no 
objective benchmark on which to base a true level, and this subjective over-reporting may. in fact, be the reality of the magnitude of the effects of disability and impairment on handicap. However, this type of recall bias would not affect the measurement of exposure since the nurse coordinator measured disability before the handicap assessment was completed.

\section{iii) Statistical Power}

Statistical power is the ability of a statistical analysis to detect differences between the groups of interest given that there really is a difference. The statistical analyses employed in this study appeared to have enough power to detect a difference between individuals with different levels of impairment and disability. According to the sample size calculations performed earlier, a minimum of 14 subjects was needed in each of the three levels of the impairment or disability variables in order to detect an effect size of 10 points on the RNL index with a probability of a Type I error at $5 \%$ and a probability of a Type II error at $20 \%$. In most of the statistical analyses, these sample size requirements were met, although there were occasionally insufficient numbers of subjects in the dependent or severely impaired subgroups, suggesting that the postulated effect size may have been larger than anticipated. For instance, in spite of less than five observations in the motor dependent subgroups, statistically significant differences were observed between the handicap scores in the independent and dependent motor disability groups, although the limited number of observations prevented the detection of differences between the moderately dependent and dependent groups at both follow-up periods.

Furthermore, there may have been important effects on handicap from other variables that could not be fully explored without a greater number of subjects. Specifically, the full extent of the interaction effect between rehabilitation therapy and motor disability at three months was uninterpretable due to a small number of subjects in the motor dependent subgroup. In order to further clarify the extent of this association, a greater number of subjects who fall into the dependent sub-groups is needed. However, further research into the validity of proxy responses or other alternative techniques will be necessary before information on handicap can be obtained from those who are more impaired or disabled. 


\section{iv) Other Limitations}

While the ICIDH can be a useful tool with which to understand the impact of illness in a person's life, one has to be careful that the classification system is not used to objectify or classify people. The classifications of the ICIDH are heuristic categories only and are not intended to characterize individuals. Indeed, this is one of the major criticisms of the ICIDH model. Some individuals with disabilities actively oppose the ICIDH because they see it as "medicalizing" disability.

It must also be recognized that while the ICIDH categories may have explained some of the social experience following stroke, over $50 \%$ of the variance in handicap was left unexplained by the statistical modelling in this research. There is no magic formula to predict handicap in all stroke survivors; each individual is unique. There were individuals in this study who had severe disabilities and impairments following their stroke, but who did not report extensive handicap. For instance, individuals with severe stroke impairment reported a 35 point improvement in their handicap score between the three month and one year follow-up periods even though they remained severely impaired.

On the other hand, some individuals with only minor impairments reported substantial poststroke handicap. For instance, one elderly gentleman in this study suffered only minor impairments due to his stroke and was able to remain completely independent in activities of daily living. However, the part of his brain that was affected by the stroke was that part that was responsible for reading and writing. Because this man was actively involved in the academic world, such impairments were devastating, and he fell into a severe depression with extensive handicap, even though his stroke left him with virtually no residual physical disability.

Therefore, while this research has been positivistic, it is important to recognize that impairments and disabilities will not always lead to handicap following illness and injury. Other personal factors unique to each individual are important to the handicap creation process. An understanding of these processes requires a more interpretive research approach. 


\section{CONCLUSION}

Stroke is becoming more disabling than lethal, with declining mortality trends creating a pool of stroke survivors with varying degrees of physical and cognitive disability. Therefore, as survival following stroke increases, it becomes less important to focus on how people can live longer, and more important to determine how people can live better with residual disability and handicap. While the emphasis of stroke outcome research has focused on the physical and selfcare aspects of recovery, it is clearly incomplete given that physical disability has been shown to be only a minor part of the life experience of stroke survivors (Becker, 1993; Gresham et al., 1975; Gresham, 1986; Labi, Phillips, \& Gresham, 1980). Health care providers define recovery in terms of physical independence, while stroke survivors emphasize the return to roles and activities that are important to them as individuals (Doolittle, 1991; Kaufman, 1988). Therefore, the biomedical frame of reference is no longer sufficient to examine the stroke experience.

The purpose of this thesis has been to explore the impact of stroke on handicap in an effort to shed light on the meaning of stroke from the individual's point of view. In spite of the identified limitations and qualifications of this study, the results have demonstrated that impairments and disabilities following stroke have a significant effect on the handicap reported by individuals. Although this may not be surprising, this is one of the first studies to actually document this finding.

Furthermore, this research has demonstrated that handicap is a measurable outcome which reflects the more social consequences of illness in the lives of stroke survivors. It has provided one of the first empirical examinations of the stroke experience using the ICIDH model, with specific attention paid to the statistical interaction effects with environmental factors using generalized linear modelling. Such an explicit study of linear interactive models has received little attention in disablement research (McDonough, Badley, \& Tennant, 1995; Rigby, Rudolfer, Badley, \& Brayshaw, 1989), let alone in stroke outcome research. This thesis has attempted to clarify these relationships in order to further a conceptual and theoretical understanding of the ICIDH model and of the meaning of chronic illness in the lives of individuals.

The results of this thesis are also useful in suggesting routes of action that may alleviate or modify the social consequences of impairments and disabilities following stroke. Perhaps the 
most important finding is that handicap in stroke survivors may be alleviated if depression is appropriately recognized and treated. Furthermore, rehabilitation therapy may be particularly effective in reducing handicap for those survivors who are moderately dependent in mobility at three months post-stroke. Family resources should also be supported, particularly for male survivors. Other social resources should be targeted to female stroke survivors, especially those who are married, as it appears that their spousal role may be detrimental to handicap. Finally, attention should be paid to issues of control in institutional environments, as this may be the reason why residence at home is beneficial for handicap. All these findings suggest ways in which social policy can be directed so as to benefit the lives of stroke survivors. With such knowledge, health professionals and policy makers can possibly prevent handicap from occurring in future stroke survivors, or target support and rehabilitation services appropriately so that the impact of stroke is minimized. 


\section{RECOMMENDATIONS FOR FUTURE RESEARCH}

In addition to gathering more information on environmental factors in order to better determine their effects on handicap, future research should also combine qualitative interpretive analyses with the more quantitative normative approach taken here, in order to better understand the impact of stroke in people's lives. Research in stroke and other chronic illnesses tends to be either qualitative or quantitative. But, in order to fully comprehend the meaning of illness to individuals, there is a need to establish a link between subjective analyses and objective measures in order to develop an understanding of the mechanisms through which disabilities become problematic for individuals and situate them within the greater socio-economic context. Through such analyses it may be possible to better understand why impairments and disabilities do not always lead to handicap. There is a growing recognition that one methodological approach alone is not sufficient to understand the complex nature of public health problems (Baum, 1995), and future research should use both quantitative and qualitative methodologies synergistically to gain the understanding necessary to assist individuals in reaching optimal health and well-being.

A comparison to age and sex matched individuals without stroke would also provide information on the stroke experience over and above the changes in social activities which accompany the overall effects of aging. This should also be a component of future research. Finally, when these relationships are better understood, it will be necessary to undertake longitudinal analyses of data to examine change in handicap over time as impairments resolve and disabilities stabilize. 


\section{REFERENCES}

Adams, R.J., Meador, K.J., Sethi, K.D., Grotta, J.C., \& Thomson, D.S. (1987). Graded neurological scale for use in acute hemispheric stroke treatment protocols. Stroke, 18, 665-669.

Ahlsio, B., Britton, M., Murray, V., \& Theorell, T. (1984). Disablement and quality of life after stroke. Stroke, 15, 886-890.

Aho, K., Harmsen, P., Hatano, S., \& et al. (1980). Cerebrovascular disease in the community: results of a WHO Collaborative study. Bulletin of the World Health Organization, 58, 113-130.

American Psychiatric Association. (1987). Diaonostic and Statistical_Manual of Mental Disorders. (3rd ed.) Washington, D.C.: American Psychiatric Association.

Anderson, T.P., Bourestom, N., Greenberg, F.R., \& Hildyard, V.G. (1974). Predictive factors in stroke rehabilitation. Archives of Physical Medicine and Rehabilitation, 55, 545-553.

Andrews, K., Brocklehurst, J.C., Richards, B., \& Laycock, P.J. (1981). The rate of recovery from stroke - and its measurement. International Rehabilitation Medicine, 3, 155-161.

Badley, E.M. (1987). The ICIDH: format, application in different settings, and the distinction between disability and handicap; a critique of papers on the application of the International Classification of Impairments, Disabilities and Handicaps. Intemational Disability Studies, 2 , 122-125.

Badley, E.M. (1993). An introduction to the concepts and classifications of the international classification of impairments, disabilities and handicaps. Disability and Rehabilitation, 15, 161-178.

Badley, E.M. (1995). The genesis of handicap: definition, models of disablement, and role of external factors. Disability and Rehabilitation, 17, 53-62.

Baum, F. (1995). Researching public health: behind the qualitative-quantitative methodological debate. Social Science and Medicine, 40, 459-468.

Becker, G. (1993). Continuity after stroke: Implications for life-course disruption in old age. The Gerontologist, 33, 148-158.

Bladin, C.F., Smurawska, L.T., Alexandrov, A.V., Zhu, C.Z., \& Norris, J.W. (1993). Left carotid dominance in stroke. Canadian Joumal of Neurological Sciences, 20, S70.

Bohannon, R.W., Kloter, K., \& Cooper, J. (1991). Recovery and outcome in patients with stroke treated in an acute care hospital. Journal of Stroke and Cerebrovascular Diseases, 1, 190-195. 
Boneau, C.A. (1960). The effects of violations of assumptions underlying the t-test. Psychological Bulletin, 57, 49-64.

Bonita, R. (1992). Epidemiology of stroke. Lancet, 339, 342-344.

Bonita, R., Anderson, A., \& North, J.D.K. (1987). The pattern of management after stroke. Age and Ageing, 16, 29-34.

Bonita, R. \& Beaglehole, R. (1993). Explaining stroke mortality trends. Lancet, 341, 1510-1511.

Bonita, R. \& Beaglehole, R. (1995). Monitoring stroke: an international challenge (editorial). Stroke, 26, 541-42.

Bonita, R., Beaglehole, R., \& North, J.D.K. (1984). Event, incidence and case fatality rates of cerebrovascular disease in Auckland, New Zealand. American Joumal of Epidemiology, 120, 236-243.

Bradburn, N.M. (1969). Structure of psychological well being. Chicago: Aldine Publishing.

Bray, G.P., DeFrank, R.S.. \& Wolfe, T.L. (1981). Sexual functioning in stroke survivors. Archives of Physical Medicine and Rehabilitation, 62, 286-288.

Brocklehurst, J.C., Morris, P., \& Andrews, K. (1981). Social effects of stroke. Social Science and Medicine, 15, 35-39.

Brott, T., Adams, H.P., Olinger, C.P., Marler, J.R., Barsan, W.G., Biller, J., Spilker, J., Holleran, R., Eberle, R., Hertzberg, V., Rorick, M., Moomaw, C.J., \& Walker, M. (1989). Measurements of acute cerebral infarction: a clinical examination scale. Stroke, 20, 864-870.

Campbell, A.J., Busby, W.J., Robertson, M.C., Lum, C.L., Langlois, J.A., \& Morgan, F.C. (1994). Disease, impairment, disability and social handicap: a community based study of people aged 70 years and over. Disability and Rehabilitation, 16, 72-79.

Canadian Society for the ICIDH. (1991). The handicaps creation process: how to use the conceptual model. ICIDH Intermational Network, 4, 11 -58.

Chambers, B.R., Norris, J.W., Shurvel, B.L., \& Hachinski, V.C. (1987). Prognosis of acute stroke. Neurology, 37, 221-225.

Chamie, M. (1989). Survey design strategies for the study of rehabilitation. World Health Statistics Quarterly, 42, 122-140. 
Chelluri, L., Pinsky, M.R., Donahoe, M.P., \& Grenvik, A. (1993). Long-term outcome of critically ill elderly patients requiring intensive care. Joumal of the American Medical Association, 269, 31 19-3123.

Christie, D. (1981). Stroke in Melbourne, Australia: an epidemiological study. Stroke, 12. 467-469.

Claris Corporation. (1993). EileMaker Pro. Santa Clara, CA: Claris Corporation.

Cleary, P.D. \& Mechanic, D. (1983). Sex differences in psychological distress among married people. Joumal of Health and Social Behaviour, 24, 111-121.

Cochrane, A.L. (1970). Burden of cerebrovascular disease. British Medical Joumal, 3, 165.

Cohen, J. (1977). Statistical Power Analysis for the Social Sciences. (2nd ed.) New York: Academic Press.

Colantonio, A., Kasl, S.V., \& Ostfeld, A.M. (1994). Level of function predicts post-stroke outcomes in the elderly. Gerontologist, 34, 254.

Cote, R., Hachinski, V.C., Shurvel, B.L., Norris, J.W., \& Wolfson, C. (1986). The Canadian Neurological Scale: a preliminary study in acute stroke. Stroke, 17, 731-737.

Data Management Service of the Uniform Data System for Medical Rehabilition. (1990). Guide For Use of the Uniform Data Set for Medical Rehabilitation (Version 3.1). Buffalo, NY: State University of New York at Buffalo.

de Haan, R., Aaronson, N., Limburg, M., Langton Hewer, R., \& van Crevel, H. (1993). Measuring quality of life in stroke. Stroke, 24, 320-327.

de Haan, R.J., Limburg, M., Van der Meulen, J.H., Jacobs, H.M., \& Aaronson, N.K. (1995). Quality of life after stroke: impact of stroke type and lesion location. Stroke, 26, 402-408.

Dejong, G. \& Branch, L.G. (1982). Predicting the stroke patient's ability to live independently. Stroke, 13, 648-655.

Desjardin, B. (1992). Population ageing and the elderly: current demographic analysis. Ottawa: Statistics Canada.

Desmeules, M., Huang, J., \& Mao, Y. (1993). Projections of deaths and hospitalizations among elderly Canadians. Chronic Diseases in Canada, 14, 145-150. 
Diehr, P., Patrick, D., Hedricks, R., Rothman, M., Grembowski, D., Raghunathan. T.E., \& Beresford, S. (1995). Including deaths when measuring health status over time. Medical Care, 33, AS164-AS172.

Dobkin, B.H. (1989). Focused stroke rehabilitation programs do not improve outcome. Archives of Neurology, 46, 701-703.

Dodds, T.A., Martin, D.P., Stolov, W.C., \& Deyo, R.A. (1993). A validation of the Functional Independence Measurement and its performance among rehabilitation inpatients. Archives of Physical Medicine and Rehabilitation, 74, 531-536.

Doolittle, N.D. (1991). Clinical ethnography of lacunar stroke: implications for acute care. Journal of Neuroscience Nursing, 23, 235-240.

Eardley, A., Cribb, A., \& Pendleton, L. (1991). Ethical issues in psychosocial research among patients with cancer. European Joumal on Cancer, 27, 166-196.

Eastwood, M.R., Rifat, S.L., Nobbs, H., \& Ruderman, J. (1989). Mood disorder following cerebrovascular accident. British Joumal of Psychiatry, 154, 195-200.

Ebrahim, S. (1990). Clinical Epidemiology of Stroke. Oxford: Oxford University Press.

Elwood, J.M. (1988). Selection of subjects for study. In Anonymous (Ed.). Causal Relationships in Medicine: A Practical System for Critical Appraisal (pp. 38-57). Toronto: Oxford University Press.

Engel, G.L. (1977). The need for a new medical model: a challenge for biomedicine. Science, 196, 129-136.

Estes, C.L. \& Rundall, T.G. (1992). Social characteristics, social structure, and health in the aging population. In M.G. Ory, R.P. Abeles \& P.D. Lipman (Eds.). Aging, Health, and Behavier (pp. 299-326). Newbury Park, CA: Sage.

Evans, R.L., Bishop, D.S., \& Haselkom, J.K. (1991). Factors predicting satisfactory home care after stroke. Archives of Physical Medicine and Rehabilitation, 72, 144-147.

Evans, R.L., Connis, R.T., Bishop, D.S., Hendricks, R.D., \& Haselkom, J.K. (1994). Stroke: a family dilemma. Disability and Rehabilitation, 16, 110-118.

Feibel, J.H. \& Springer, C.J. (1982). Depression and failure to resume social activities after stroke. Archives of Physical Medicine and Rehabilitation, 63, 276-278. 
Ferruci, L.. Bandinelli, S., Guralnik. J.M., Lamponi, M., Bertini, C., Falchini, M., \& Baroni, A. (1993). Recovery of functional status after stroke: A post-rehabilitation follow-up study. Stroke, 24, 200-205.

Foulkes, M.A., Wolf, P.A., Price, T.R., Mohr, J.P., \& Hier, D.B. (1988). The Stroke Data Bank: design, methods, and baseline characteristics. Stroke, 19, 547-554.

Gaito, J. (1980). Measurement scales and statistics: resurgence of an old misconception. Psychological_Bulletin, 87, 564-567.

Galski, T., Bruno, R.L., Zorowitz, R., \& Walker, J. (1993). Predicting length of stay, functional outcome, and aftercare in the rehabilitation of stroke patients: the dominant role of higher-order cognition. Stroke, 24, 1794-1800.

Goleman, D. (1995). Treat depression to help elderly, U.S. study says. Globe and Mail, September 6.1995, A6.

Gordon, M. (1993). Monograph series on aging-related diseases: III. Stroke (Cerebrovascular Disease). Chronic Diseases in Canada, 14, 64-89.

Gove, W.R. (1978). Sex differences in mental illness among adult men and women. Social Science and Medicine, 12B, 187-198.

Gove, W.R., Hughes, M., \& Style, C.B. (1983). Does marriage have positive effects on the psychological well-being of the individual?. Joumal of Health and Social Behaviour, 24, 122-131.

Gove, W.R. \& Tudor, J.F. (1973). Adult sex roles and mental illness. American Jourmal of Sociology, 28, 812-835.

Gowland, C. (1982). Recovery of motor function following stroke: profile and predictors. Physiotherapy Canada, 34, 77-84.

Granger, C.V., Cotter, A.C., Hamilton, B.B., \& Fiedler, R.C. (1993). Functional assessment scales: A study of persons after stroke. Archives of Physical Medicine and Rehabilitation, 74, 133-138.

Granger, C.V. \& Gresham, G.E. (1990). International Classification of Impairments, Disabilities and Handicaps (ICIDH) as a conceptual basis for stroke outcome research: A tribute to Philip H.N. Wood. Stroke, 2l(suppl II), II66-II67.

Granger, C.V. \& Hamilton, B.B. (1990). Measurement of stroke rehabilitation outcome in the 1980s. Stroke, 21(Suppl 2), II46-II47. 
Granger, C.V., Hamilton, B.B., Gresham, G.E., \& Kramer, A.A. (1989). The Stroke Rehabilitation Outcome Study Part II: Relative merits of the total Barthel Index score and a four-item subscore in predicting patient outcomes. Archives of Physical Medicine and Rehabilitation, 70, 100-103.

Granger, C.V., Hamilton, B.B., Keith, R.A., Zielezny, M. \& Sherwin, F.S. (1986). Advances in functional assessment for rehabilitation. In Anonymous (Ed.). Topics in Geriatric Rehabilitation (pp. 59-74). Rockville, MD: Aspen.

Granger, C.V., Hamilton, B.B., Linacre, J.M., Heinemann, A.W., \& Wright, B.D. (1993). Performance profiles of the functional independence measure. American Joumal of Physical Medicine and Rehabilitation, 22, 84-89.

Gresham, G.E., Fitzpatrick, T.E., Wolf, P.A., McNamara, P.M., Kannel, W.B., \& Dawber, T.R. (1975). Residual disability in survivors of stroke - The Framingham Study. New England Joumal of Medicine, 293, 954-956.

Gresham, G.E. (1986). The rehabilitation of the stroke survivor. In H.J.M. Bamett, B.M. Stein, J.P. Mohr \& F.M. Yatsu (Eds.). Stroke: Pathophysiology_Diagnosis and Management (2nd ed.) New York: Churchill Livingstone.

Gresham, G.E. (1990). Past achievements and new directions in stroke outcome research. Proceedings of a National Symposium on Methodological Issues in Stroke Outcome Research. Stroke, 21(suppl LI), II1-II2.

Gresham, G.E., Philips, F., \& Wolf, P.A. (1979). Epidemiologic profile of long-term stroke disability: The Framingham Study. Archives of Physical Medicine and Rehabilitation, 60, 487-491.

Grimby, G., Eriksson, B.G., Frandin, K., Grimby, A., Gustavsson, G., Mellstrom, D., \& Sonn, U. (1993). On the use of the WHO handicap classification in a population study of 73- and 76-year-old men and women. Disability and Rehabilitation, 15, 107-113.

Hamilton, B.B. \& Granger, C.V. (1994). Disability outcomes following inpatient rehabilitation for stroke. Physical Therapy, 74, 494-503.

Hamilton, B.B., Laughlin, J.A., Granger, C.V., \& Kayton, R.M. (1991). Interrater agreement of the seven level Functional Independence Measure (FIM). Archives of Physical Medicine and Rehabilitation, 72, 790.

Harwood, R.H., Gompertz, P., \& Ebrahim, S. (1994). Handicap one year after a stroke: validity of a new scale. Joumal of Neurology. Neurosurgery and Psychiatry, 57, 825-829. 
Hayashi, H., Ahiko, T., \& Yasumura, S. (1995). Factors related to survival rate, and life satisfaction in stroke patients in Yamagata Prefecture, Japan. Japanese Joumal of Public Health, 42, 19-30.

Heart and Stroke Foundation of Ontario. (1994). (un pub)

Hebert, R., Carrier, R, \& Bilodeau, A. (1988). The Functional Autonomy Measurement System (SMAF): Description and validation of an instrument for the measurement of handicaps. Age and Aging, 17, 293-302.

Heinemann, A.W., Linacre, J.M., Wright, B.D., Hamilton, B.B., \& Granger, C.V. (1993). Relationships between impairment and physical disability as measured by the Functional Independence Measure. Archives of Physical Medicine and Rehabilitation, 74, 566-573.

Henley, S., Pettit, S., Todd-Pokropek, A., \& Tupper, A. (1985). Who goes home? Predictive factors in stroke recovery. Joumal of Neurology. Neurosurgery and Psychiatry, 48, 1-6.

Hennekens, C.H. \& Buring, J.E. (1987). Epidemiology in Medicine. Boston: Little, Brown and Company.

Hier, D.B. (1983). Behavioural abnormalities after right hemisphere stroke. Neurology, 33 , 337-344.

Hirdes, J.P. \& Forbes, W.F. (1992). The importance of social relationships, socioeconomic status and health practices with respect to mortality among healthy Ontario males. Joumal of Clinical Epidemiology, 45, 175-182.

Jecker, N.S. (1991). Age-based rationing and women. Joumal of the American_Medical Association, 266, 3012-3015.

Johansson, B.B., Jadback, G., Norrving, B., Widner, H., Wiklund, L., \& Wiklund, I. (1992). Evaluation of long-term functional status in first-ever stroke patients in a defined population. Scandanavian Joumal of Rehabilitation Medicine, 26(suppl), 105-114.

Kandel, D.B., Davies, M., \& Raveis, V.H. (1985). The stressfulness of daily social roles for women: marital, occupational and household roles. Joumal of Health and Social Behaviour, 26, 64-78.

Kannel, W.B. (1971). Current status of the epidemiology of brain infarction associated with occlusive arterial disease. Stroke, 2, 295-318. 
Kaufman, S.R. (1988). Stroke rehabilitation and the negotiation of identity. In S. Reinharz \& G.D. Rowles (Eds.). Qualitative Gerontology (pp. 82-103). New York: Springer Publishing Company.

Keith, R.A., Granger, C.V., Hamilton, B.B. \& Sherwin, F.S. (1987). The Functional Independence Measure: a new tool for rehabilitation. In M.G. Eisenberg \& R.C. Grzesiak (Eds.). Advances in Clinical Rehabilitation (pp. 6-18). New York: Springer.

Kelly-Hayes, M. (1990). Time intervals, survival and destination: three crucial variables in stroke outcome research. Stroke, 21(suppl 2), II24-II26.

Kempen, G.I.J.M. \& Suurmeijer, T.P.B.M. (1991). Professional home care for the elderly. Society for Scientific Medicine, 33, 1081-1089.

Kertesz, A. \& Black, S.E. (1985). Cerebrovascular disease and aphasia. In J.K. Darby (Ed.). Speech and Lanquage Evaluation in Neurology Orlando, Florida: Grune \& Stratton.

Kessler, R. \& Essex, M. (1982). Marital status and depression: the importance of coping resources. Social Forces, 61, 484-507.

Kilner, J.F. (1989). Age criteria in medicine: are the medical justifications ethical?. Archives of Intemal Medicine, 149, 2343-2346.

Kimura, A., Chino, N., Saitoh, E., Sonoda, S., \& Domen, K. (1994). An evaluation of stroke patients in rehabilitation medicine. Keio Joumal of Medicine, 43, 143-148.

Korner-Bitensky, N. (1993). A telephone interview compared to a face-to-face interview in determining health status of patients discharged home from rehabilitation hospital. McGill University, Montreal: PhD Thesis, School of Physical and Occupational Therapy.

Kotila, M., Waltimo, O., \& Marjaiisa, N. (1984). The profile of recovery from stroke and factors influencing outcome. Stroke, 15, 1039.

Kurtzke, J.F. (1986). Stroke: geographical variation. In F. Clifford Rose (Ed.). Stroke: Epidemiological. Therapeutic and Socio-economic Aspects (pp. 11-24). London: Royal Society of Medicine Services Limited.

La Rosa, F., Celani, M.G., Duca, E., Righetti, E., Saltalamacchia, G., \& Ricci, S. (1993). Stroke care in the next decades: a projection derived from a community-based study in Umbria, Italy. European Joumal of Epidemiology, 2, $151-154$. 
Labi, M.L.C., Phillips, T.F., \& Gresham, G.E. (1980). Psychosocial disability in physically restored long-term stroke survivors. Archives of Physical Medicine and Rehabilitation, 61, 561-565.

Linacre, J.M., Heinemann, A.W., Wright, B.D., Granger, C.V. \& Hamilton, B.B. (1991). The Functional Independence Measure as a measure of disability (Research Report 91-01). Chicago: Rehabilitation Services Evaluation Unit, Rehabilitation Institute of Chicago.

Lipsey, J.R., Robinson, R.G., \& Pearlson, G.D. (1984). Nortriptyline treatment for post-stroke depression: a double-blind trial. Lancet, I, 297-300.

Longino, C.F., Warheit, G.J. \& Green, J.A. (1989). Class, aging and health. In K.S. Markides (Ed.). Agine and Health: Perspectives on Gender. Race. Ethnicity and Class (pp. 79-109). Newbury Park, CA: Sage.

Manolio, T.A. \& Furberg, C.D. (1992). Age as a predictor of outcome: what role does it play?. American Joumal of Medicine, 22, 1-6.

Mayo, N.E., Korner-Bitensky, N.A., \& Becker, R. (1991). Recovery time of independent function post-stroke. American Joumal of Physical Medicine and Rehabilitation, 70, 5-12.

McDonough, P.A., Badley, E.M., \& Tennant, A. (1995). Disability, resources, role demands and mobility handicap. Disability and Rehabilitation, 17, 159-168.

McMullen, P.A. \& Gross, A.E. (1983). Sex differences, sex roles, and health-related help-seeking. In B.M. DePaulo, A. Nadler \& J.D. Fisher (Eds.). New Directions in Helping: Vol 2. Help Seeking (pp. 233-263). New York: Academic Press.

Microsoft Corporation. (1994). Microsoft Excel Version 5.0. Seattle, WA: Microsoft Corporation.

Millikan, C.H., McDowell, F. \& Easton, J.D. (1987a). Stroke. Philadelphia: Lea and Febiger.

Millikan, C.H., McDowell, F. \& Easton, J.D. (1987b). The Epidemiology of Stroke. Philadelphia: Lea \& Febiger.

Muhlenkamp, A.F. \& Sayles, J.A. (1986). Self-esteem, social support and positive health practices. Nursing Research, 35, 33-37.

Norman, G.R. \& Streiner, D.L. (1994). Biostatistics; the Bare Essentials. St. Louis, MO: Mosby. 
Oczkowski. W.J. \& Barreca, S. (1993). The Functional Independence Measure: Its use to identify rehabilitation needs in stroke survivors. Archives of Physical Medicine and Rehabilitation, 74, 1291-1294.

Oxfordshire Community Stroke Project. (1983). Incidence of stroke in Oxfordshire: first years experience of a community stroke register. British Medical Joumal, 287, 713-717.

Parikh, R.M., Robinson, R.G., Lipsey, J.R., Starkstein, S.E., Fedoroff, J.P., \& Price, T.R. (1990). The impact of post-stroke depression on recovery in activities of daily living over a two year follow-up. Archives of Neurology, 47, 785-789.

Partridge, C.J., Johnston, M., \& Edwards, S. (1987). Recovery from physical disability after stroke: normal patterns as a basis for evaluation. Lancet, 373-375.

Perry, J., Garrett, M., Gronley, J.K., \& Mulroy, S.J. (1995). Classification of walking handicap in the stroke population. Stroke, 26, 982-989.

Petrasovits, A. \& Nair, C. (1994). Epidemiology of stroke in Canada. Health Reports, 6(1), 39-44.

Pierre, U., Korner-Bitensky, N., Hanley, J., \& Wood-Dauphinee, S. (1995). Proxy use of the SF-36 in rating health status of the elderly. Quality of Life Research, 4, 473.

Rasch, G. (1980). Probabilistic models for some intelligence and attainment tests. Chicago: University of Chicago.

Reding, M.J., Orto, L.A., \& Winter, S.W. (1986). Antidepressant therapy after stroke: a double-blind trial. Archives of Neurology, 43, 763-765.

Richards, C., Malouin, F., Wood-Dauphinee, S.L., Williams, J.I., Bouchard, J.P., \& Brunet, D. (1993). Task specific physical therapy for optimization of gait recovery in acute stroke patients. Archives of Physical Medicine and Rehabilitation, 74, 612-620.

Rigby, A.S., Rudolfer, S.M., Badley, E.M., \& Brayshaw, N.C. (1989). The relationship between impairment and disability in arthritis: an application of the theory of generalized linear models to the ICIDH. International Disability Studies, $\mathbf{L}, \mathbf{8 4 - 8 8}$.

Robinson, R.G., Bolla-Wilson, K., Kaplan, E., Lipsey, J.R., \& Price, T.R. (1986). Depression influences intellectual impairment in stroke patients. British Journal of Psychiatry, 48, 541-547.

Robinson, R.G., Kubos, K.L., Starr, L.B., Rao, K., \& Price, T.R. (1984). Mood disorders in stroke patients: Importance of location of lesion. Brain, 107, 81-93. 
Robinson, R.G. \& Starkstein, S.E. (1990). Current research in affective disorders following stroke. Joumal of Neuropsychiatry, 2, 1-14.

Robinson, R.G., Starr, L.B., Kubos, K.L., \& Price, T.R. (1983). A two year longitudinal study of post-stroke mood disorders: findings during the initial evaluation. Stroke, 14, 736-741.

Rosner, B. (1990). Bartlett's test for homogeneity of variance. In B. Rosner (Ed.). Eundamentals of Biostatistics (3rd ed.) (pp. 495-498). Belmont, Ca.: Duxbury Press.

Sacco, R.L., Wolf, P.A., Kannel, W.B., \& McNamara, P.M. (1982). Survival and recurrence following stroke: The Framingham Study. Stroke, 13, 290-295.

SAS Institute Inc. (1985a). SAS Language Guide for Personal Computers. Version 6 Edition. Cary, N.C.: SAS Institute Inc..

SAS Institute Inc. (1985b). SAS Procedure Guide for Personal Computers, Version 6 Edition. Cary, N.C.: SAS Institute Inc..

Schlesselman, J.J. (1982). Case-Control Studies: Design.Conduct Analysis. New York: Oxford University Press.

Seale, C. \& Davies, P. (1987). Outcome measurement in stroke rehabilitation research. International Disability Studies, $2,155-160$.

Shahar, E., McGovern, P.G., Sprafka, M., Pankow, J.S., Doliszny, K.M., Luepker, R.V., \& Blackburn, H. (1995). Improved survival of stroke patients during the 1980s: the Minnesota Stroke Survey. Stroke, 26, 1-6.

Silliman, R.A., Wagner, E.H., \& Fletcher, R.H. (1987). The social and functional consequences of stroke for elderly patients. Stroke, 18, 200-203.

Simonsick, E.M., Wallace, R.B., Blazer, D.G., \& Berkman, L.F. (1995). Depressive symptomatology and hypertension-associated morbidity and mortality in older adults. Psychosomatic Medicine, 57, 427-435.

Sinyor, D., Amato, P., Kaloupek, D.G., Becker, R., Goldenberg, M., \& Coopersmith, H. (1986). Post-stroke depression: relationship to functional impairment, coping strategies and rehabilitation outcome. Stroke, 17, 1102-1107.

Skilbeck, C.E., Wade, D.T., Hewer, R.L., \& Wood, V.A. (1983). Recovery after stroke. Joumal of Neurology. Neurosurgery and Psychiatry, 46, 5-8. 
Smith. D.S., Goldenberg, E., \& Ashburn, A. (1981). Remedial therapy after stroke: a randomised controlled trial. British Medical Joumal, 282, 517-520.

Smurawska, L.T., Alexandrov, A.V., Bladin, C.F., \& Norris, J.W. (1994). Cost of acute stroke care in Toronto, Canada. Stroke, 25, 1628-1631.

Sneeuw, K.C.A., Aaronson, N.K., Osoba, D., Muller, M.J., Hsu, M.A., Yung, W.K.A., Brada, M., \& Newlands, E.S. (1995). The use of significant others as proxy raters of the quality of life of patients with brain cancer. Quality of Life Research, 4, 486-487.

Soderback, I. \& Ekholm, J. (1992). Medical and social factors affecting behaviour patterns in patients with acquired brain damage: a study of patients living at home three years after the incident. Disability and Rehabilitation, 14, 30-35.

Solomon, N.A., Glick, H.A., Russo, C.J., Lee, J., \& Schulman, K.A. (1994). Patient preferences for stroke outcome. Stroke, 25, 1721-1725.

Spitzer, W.O., Dobson, A.J., Hall, J., \& Chesterman, E. (1981). Measuring quality of life in cancer patients: a concise QL-Index for use by physicians. Joumal of Chronic Diseases, 34, 585-598.

Starkstein, S.E., Parikh, R.M., \& Robinson, R.G. (1987). Post-stroke depression and recovery after stroke. Lancet, I, 743.

Starkstein, S.E. \& Robinson, R.G. (1989). Affective disorders and cerebral vascular disease. British Joumal of Psychiatry, 14, 170-182.

Starkstein, S.E. \& Robinson, R.G. (1993). Depression in cerebrovascular disease. In S.E. Starkstein \& R.G. Robinson (Eds.). Depression in Neurologic Disease (pp. 28-49). Baltimore: The Johns Hopkins University Press.

Statistics Canada. (1992). Health Reports: Hospital Morbidity 1989-1990. (4th ed.) Ottawa: Minister of Industry, Trade and Technology.

Stevens, R.S., Ambler, N.R., \& Warren, M.D. (1984). A randomized controlled trial of a stroke rehabilitation ward. Age and Ageing, 13, 65-75.

Stroebe, M.S. \& Stroebe, W. (1983). Who suffers more? Sex differences and psychological risks of the widowed. Bsychological Bulletin, 23, 279-303.

Sugisawa, H. (1991). Treatment compliance and subjective well-being among post-stroke patients. Japanese Journal of Public Health, 38, 70-78. 
Sunnybrook Health Science Centre. (1992). Sunnybrook Health Science Centre Catchment Area Demographic and Social Indicators. (un pub)

Task Force on Stroke Handicap. (1990). Symposium recommendations for methodology in stroke outcome research. Stroke, 21(suppl III, II68-II73.

Thoits, P.A. (1982). Life stress, social support and psychological stress: a reformulation and test of the social isolation hypothesis. American Joumal of Community Psychology, 10, 341-362.

Thoits, P.A. (1983). Multiple identities and psychological well-being. American Sociological Review, 48, 174-187.

Thoits, P.A. (1985). Social support and psychological well-being: theoretical possibilities. In I.G. Sarason \& B.R. Sarason (Eds.). Social Support: Theory, Research and Applications. Proceedings of the NATO Advances Research Workshop on Social Support (pp. 51-72). Dordrecht: Martinus Nijhoff Publishers.

Veloso, F. (1994). Cost and cost-containment of stroke. Health Reports, 60Ne.1 Addendum), 20.

Verbrugge, L.M. (1983). Multiple roles and physical health of women and men. Joumal of Health and Social Behaviour, 24, 16-30.

Verbrugge, L.M. (1984). Longer life but worsening health? Trends in health and mortality of middle aged and older persons. Milbank Memorial Fund Quarterly, 62, 475-519.

Verbrugge, L.M. \& Jette, A.M. (1993). The disablement process. Social Science and Medicine, $38,1-14$.

Verbrugge, L.M., Lepkowski, J.M., \& Imanaka, Y. (1989). Comorbidity and its impact on disability. Milbank Memorial Fund Quarterly, 67, 450-483.

Wade, D.T. \& Hewer, R.L. (1987). Functional abilities after stroke: measurement, natural history and prognosis. Joumal of Neurology. Neurosurgery and Psychiatry, 50, 177-182.

Wade, D.T., Hewer, R.L., \& Wood, V.A. (1983). The hemiplegic arm after stroke: measurement and recovery. Łoumal of Neurology Neurosurgery and Psychiatry, 46, 521-524.

Weingarten, H.R. (1985). Marital status and well-being. Joumal of Marriage and the Family, 47-55. 
Whiteneck, G.G., Charlifue, S.W., Gerhart, K.A., Overholser, J.D., \& Richardson. G.N. (1992). Quantifying handicap: a new measure of long term rehabilitation outcomes. Archives of Physical Medicine and Rehabilitation, 73, 519-526.

WHO MONICA Project, P. (1988). The World Health Organization MONICA Project (monitoring trends and determinants in cardiovascular disease): a major international collaboration. JoumaL of Clinical Epidemiology, 41, 105-114.

Wilson, M.A., Howe, D.M., \& Keith, R.A. (1991). Stroke rehabilitation: a model predicting returning to home. Westem Joumal of Medicine, 154, 587-590.

Wolf, P.A., D'Agostino, R.B., O'Neal, M.A., Sytkowski, P., Kase, C.S., Belanger, A.J., \& Kannel, W.B. (1992). Secular trends in stroke incidence and mortality: The Framingham Study. Stroke, 23, 1551-1555.

Woo, J., Kay, R., Yuen, Y.K., \& Nicholls, M.G. (1992). Factors influencing long-term survival and disability among three-month stroke survivors. Neuroepidemiology, $11,143-150$.

Wood-Dauphinee, S.L., Shapiro, S., Bass, E., Fletcher, C., Georges, P., Hensby, V., \& Mendelsohn, B. (1984). A randomized trial of team care following stroke. Stroke, 14, 864-872.

Wood-Dauphinee, S.L., Opzoomer, M.A., Williams, J.I., Marchand, B., \& Spitzer, W.O. (1988). Assessment of global function: The Reintegration to Normal Living Index. Archives of Physical Medicine and Rehabilitation, 69, 583-590.

Wood-Dauphinee, S.L. \& Williams, J.I. (1987). Reintegration to normal living as a proxy to quality of life. Joumal of Chronic Diseases, 40, 491-499.

World Health Organization. (1977). Manual of the International Statistical Classification of Diseases, Iniuries and Causes of Death. Geneva, Switzerland: World Health Organization.

World Health Organization. (1980). International Classification of Impaiments. Disabilities and Handicaps: A Manual of Classification Relating to the Consequences of Disease. First Printing. Geneva, Switzerland: World Health Organization.

World Health Organization. (1993). Foreword to the 1993 English Reprint of the ICIDH. ICIDH International Network, 6(2), 9-27.

Zung, W.W.K. (1965). A self-rating depression scale. Archives of General Psychiatry, 12 , 67-70.

Zung, W.W.K. (1990). The role of rating scales in the identification and management of the depressed patient in the primary care setting. Joumal of Clinical Psychiatry, 51(6. suppl), 72-76. 
Zung, W.W.K., Broadhead, W.E., \& Roth, M.E. (1993). Prevalence of depressive symptoms in primary care. Loumal of Family Practice, 37, 337-344.

Zung, W.W.K., Magruder-Habib, K., Valez, R., \& Alling, W. (1990). The comorbidity of anxiety and depression in general medical patients: a longitudinal study. Joumal of Clinical Psychiatry, 5166. suppl), 77-80. 


\section{APPENDIX 1}

\section{DESCRIPTION OF CLINICAL STUDY}




\title{
DESCRIPTION OF CLINICAL STUDY
}

\author{
Stroke Recovery: A Longitudinal Clinical Perfusion Correlation Study \\ S.E. Black, L. Ehrlich, C. Caldwell, J.P. Szalai \\ Sunnybrook Health Science Centre, Toronto
}

This longitudinal study was undertaken by Dr. Sandra Black and colleagues at Sunnybrook Health Science Centre between August 1990 and July 1994 with grant support from the Heart and Stroke Foundation of Ontario.

\section{Objective:}

The general objective of the study was to correlate measures of physical and neurocognitive deficits associated with hemispheric stroke with lesion parameters obtained from brain imaging techniques (CT and SPECT) in order to better understand the effects of these findings on clinical deficit and recovery. A secondary objective was to "to assess the overall quality of life and the impact of stroke on the patient's day to day social functioning".

\section{Subject Selection:}

Consecutive hemispheric stroke patients admitted to Sunnybrook Health Science Centre from August 1, 1990 to May 31, 1993 were eligible for inclusion in the study. Consenting subjects were interviewed in person at admission (in order to obtain acute and pre-morbid data), at three months and at one year post stroke. Study end points were one year completed follow-up, death or re-stroke, in which case subjects were re-enrolled in the study from the beginning.

\section{Variables:}

The variables that were collected relating to the thesis component are outlined in the chart attached. 


\section{VARIABLES COLLECTED IN STUDY}

\section{Pre-Stroke}

(Collected on Admission) Admission Three Months One Year

\section{Impairment}

Hemispheric Stroke Scale

Depression

Concomitant Illness

Side/Type of Lesion

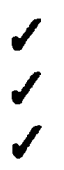

Disability

Functional Ability (FIM)

Handicap

RNL

\section{Additional Variables}

Pre-Stroke Quality of Life (Spitzer)

Use of Rehab

Living Arrangements

Demographic Factors

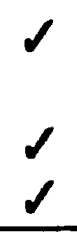

Admission Three Months One Year

$\checkmark$

$\begin{array}{ll}1 & 2 \\ 1 & 1 \\ 1 & 1 \\ 1 & 1\end{array}$

\section{$\checkmark$}

$\checkmark$

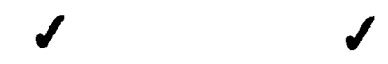




\section{APPENDIX 2}

\section{REINTEGRATION TO NORMAL LIVING INDEX}

Reprinted by permission of the publisher from

(Wood-Dauphinee S, Williams JI. Reintegration to normal living as proxy to quality of life)

Journal of Chronic Diseases, Vol 40(6), pp. 491-499.

Copyright 1987 by Elsevier Science Inc. 


\section{STROKE STUDY}

\section{REINTEGRATION TO NORMAL LIFE OUESTIONAIRE}

PATIENTI NOE.:

COCEIO

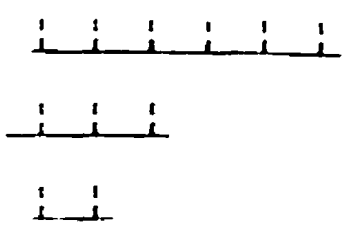

OIAGNOSIS:

UIAG

EVALP

3. 12 Months

1. 1 Month
2. 3 Moriths

SOURCE OF INFORMATION:

i. Fálenc

2. Caretaher

DATE OF EVALUATION:
DATE
3. Other

SOURCE

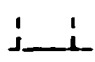

) On a scaie frun i lo ic, indicate how each of the foliowing statements applies to you. note that $i=$ does not apply while 10 = fully appiies.

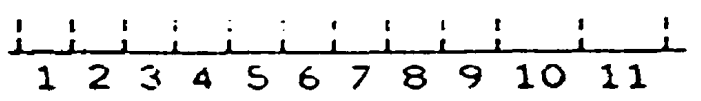

1. I move around iny iiving quarters as I feel necessary

2. I move arcund hy cummunity as I feil is necessary

3. I am abie to take trips out of town as I feel necessary

4. I am comfortable with how my self-care needs (dressing, feeding, toileting, bathing) are met

5. I spend most of my days occupied in a work activity that is necessary or importane tu me.

E. I am aole to participate in recreational activities as I want to

7 . I participate ir social activities with family. frienos and/or business acquantarices as necessary or desiratie to me.

is. I assume a role in my family which meets my needs and those of other family members.

$\Leftrightarrow$. In general. I all confortable with my personal relationsinips

io. In generai. I am comfortable with myself when I am in the compapy of others il. I feei that i.can deal with life events as they happen

TOTAL SCORE

RNLOTOT i_i 


\section{APPENDIX 3}

\section{ADAMS' HEMISPHERIC STROKE SCALE}

Reproduced with permission

(Adams RJ, Meador KF, Sethi KD, Grotta JC, Thomson DS. Graded neurologic scale for use in acute hemispheric stroke treatment protocols. Stroke 1987;18:665-669.)

Copyright 1987 American Heart Association 


\begin{tabular}{|c|c|c|}
\hline $2 / 92$ & \multicolumn{2}{|c|}{ 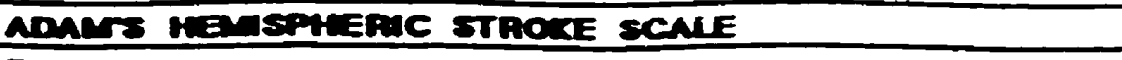 } \\
\hline \multirow{2}{*}{ SS: } & \multicolumn{2}{|c|}{ DATE } \\
\hline & \multicolumn{2}{|l|}{ men } \\
\hline 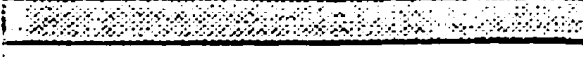 & \multicolumn{2}{|l|}{ sessoore sunenir } \\
\hline 1. Level of Consciousness & 3A. VIsual Flalds & 4A. Am \\
\hline 2. Language & 3B. Gaze & 4A. Leg \\
\hline 3. Other Cort. Functions & 3C. Face & 4E. Galt \\
\hline 4. Molor & 30. N Dysphagt & \multirow{2}{*}{$\begin{array}{l}\text { Homiparesis Tolat } \\
|4 A(A m+L e g)+3 C|\end{array}$} \\
\hline S. Sensory & 3E. Neglect & \\
\hline Tolal & 3F. Constuction & \multirow{2}{*}{$\begin{array}{l}\text { Molor Tolal } \\
\mid 4 A(A m+\operatorname{Lg})+4 E+3 C)\end{array}$} \\
\hline Severty $1(0-20) \quad 2 \quad(20-50)$ & $3(250)$ & \\
\hline \multicolumn{2}{|c|}{ NUMBER OF UNASSESSABLE ITEMS (UST) } & \multirow{2}{*}{$\begin{array}{l}\text { Motor Funcion Total } \\
\mid 4+3 C\end{array}$} \\
\hline \multicolumn{2}{|l|}{ UNASSESSABLE POINTS (TotaI) } & \\
\hline \multicolumn{2}{|c|}{ 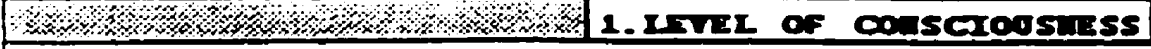 } & 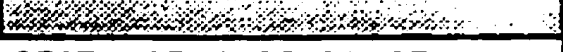 \\
\hline \multirow{7}{*}{$\begin{array}{l}\text { EYES OPEN } \\
\text { i. none } \\
\text { 2. } 10 \text { pain } \\
\text { 3. 10 speech } \\
\text { 4. spontaneously }\end{array}$} & BEST RESPONSE & EEST MOTOR RESPONSE \\
\hline & 1. none & 1. none \\
\hline & 2. Incomprohansble & 2. extonston to pain \\
\hline & 3. Imppropriate & 3. Inxion w pain \\
\hline & 4. contused & 4. fiexton whidawal \\
\hline & \multirow[t]{2}{*}{ 5. ortented } & 5. bealize pain \\
\hline & & 6. ovys commands \\
\hline 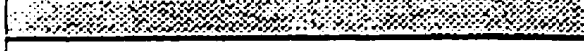 & 2. WNGINE & K \\
\hline \multirow{4}{*}{$\begin{array}{l}\text { A) COMPREHENSION } \\
\text { 1. stkK out tongue I close eyes } \\
\text { 2. polnt to door } \\
\text { 3. put il hand on fll ear then ill kree }\end{array}$} & BINANING & \multirow{4}{*}{$\begin{array}{l}\text { CI AEPETTION } \\
\text { 1. single word - dog or al } \\
\text { 2. The prosiden liwes in Washingion } \\
\text { 3. 'no its ands or bus' }\end{array}$} \\
\hline & 1. watch or bell & \\
\hline & 2. watchband or bell buekde & \\
\hline & 3. Index or ring finger & \\
\hline
\end{tabular}

$3=0,12=2,1=4$, none $=5$

$3=0,12=3,1=4$, none $=5$

$B=0,12=2,11=4$, none $=5$

OIFLUENCY Score the patbent's sponkaneous speech fiuncy or have him name as many words as he can whiln one minute which begth with the lener ' $A$ ' - (no proper names)

:sring: $>10=0,5-10=1,1-4=3$, no verbal output $=5$

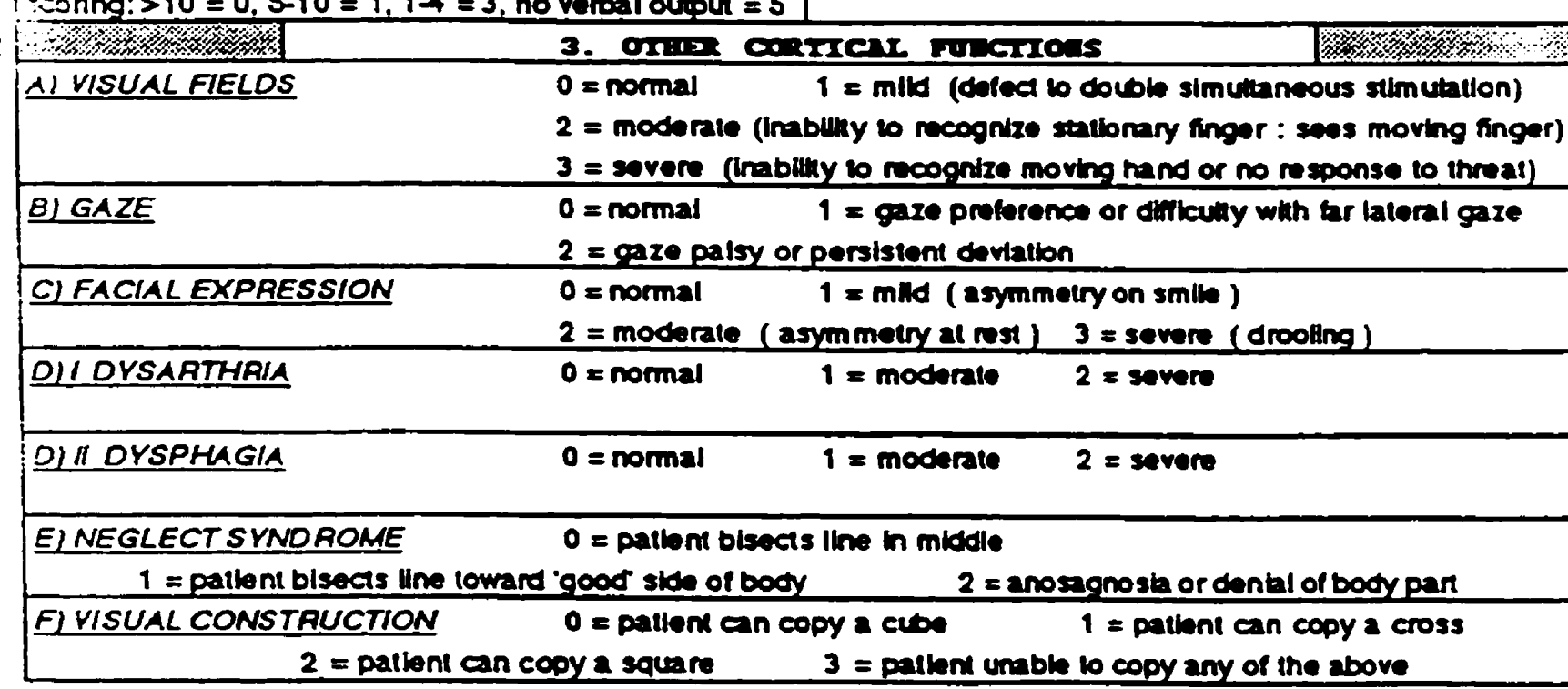

L $\mathbf{2}$ Domy

ACUTE

in

30

19

\%, 2 \%

Eyes Open Score

Best Response Score

Best Motor Response

score

GCS (3-15)

Inveried GCS

(15. Above)

112

Comprehenston Score

Naming Score

Repatition Score

Fluency Score

Loming

striot

120

Visual Fialds Score

Gaze Score

Factal Expresson

Score

Dysarthra score

Oysphagta score

Neglect Score

Visual Construction

score

Orier Conten Fone-

roos serocel

117 


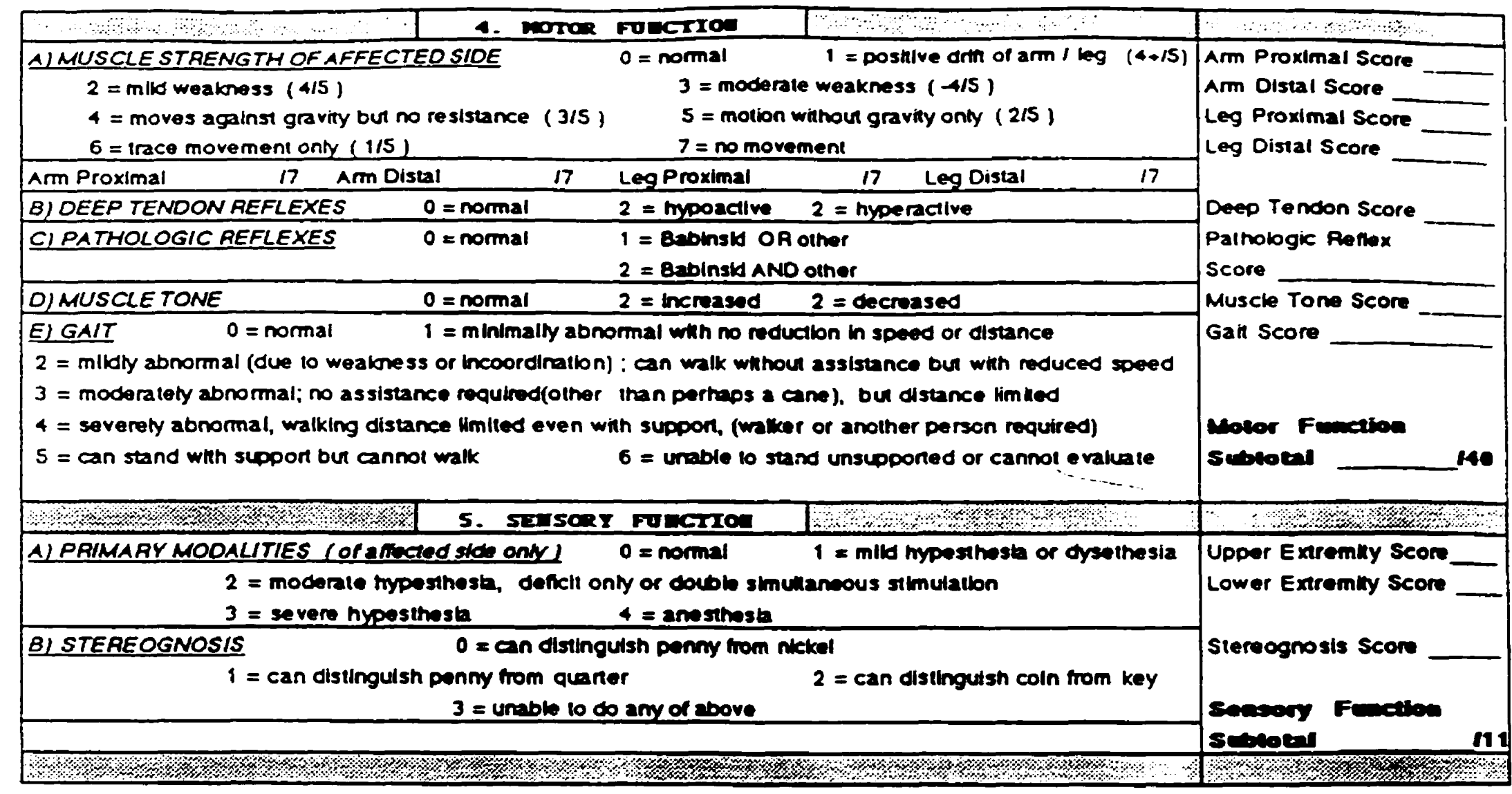

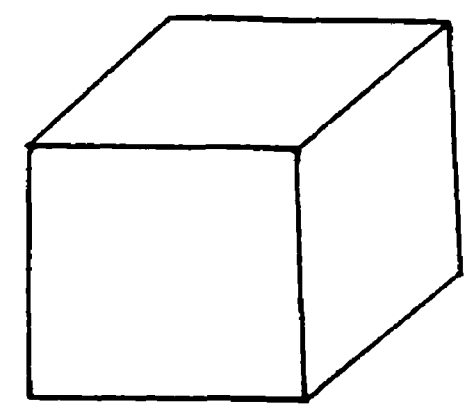




\section{APPENDIX 4}

\section{ZUNG SELF-RATING DEPRESSION SCALE}

Reproduced with permission from the Publisher

(Zung WWK, A Self-Rating Depression Scale. Archives of General Psychiatry 1965;12:63-70.)

Copyright 1965 American Medical Association. 


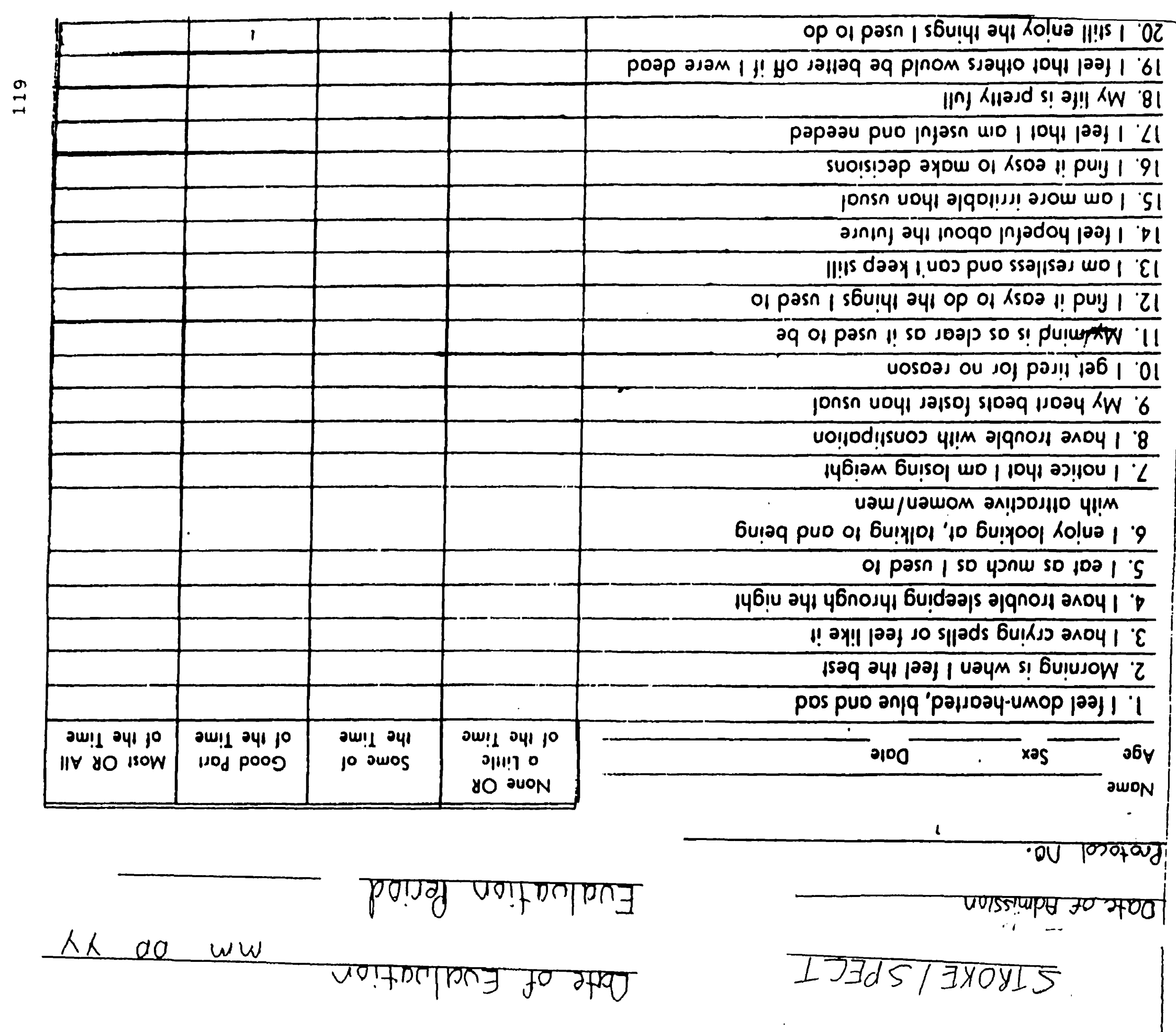




\section{APPENDIX 5}

\section{FUNCTIONAL INDEPENDENCE MEASURE}

Functional Independence Measure, Copyright 11993 Uniform Data System for Medical Rehabilitation (UDS ${ }_{M R}{ }^{\mathrm{SM}}$ ). All rights reserved. Reprinted with permission of the UDS ${ }_{\mathrm{MR}}$, University at Buffalo, 232 Parker Hall, 3435 Main Street, Buffalo, NY 14214. 
FUNCTIONAL INDEPENDENCE MEASURE

FIM

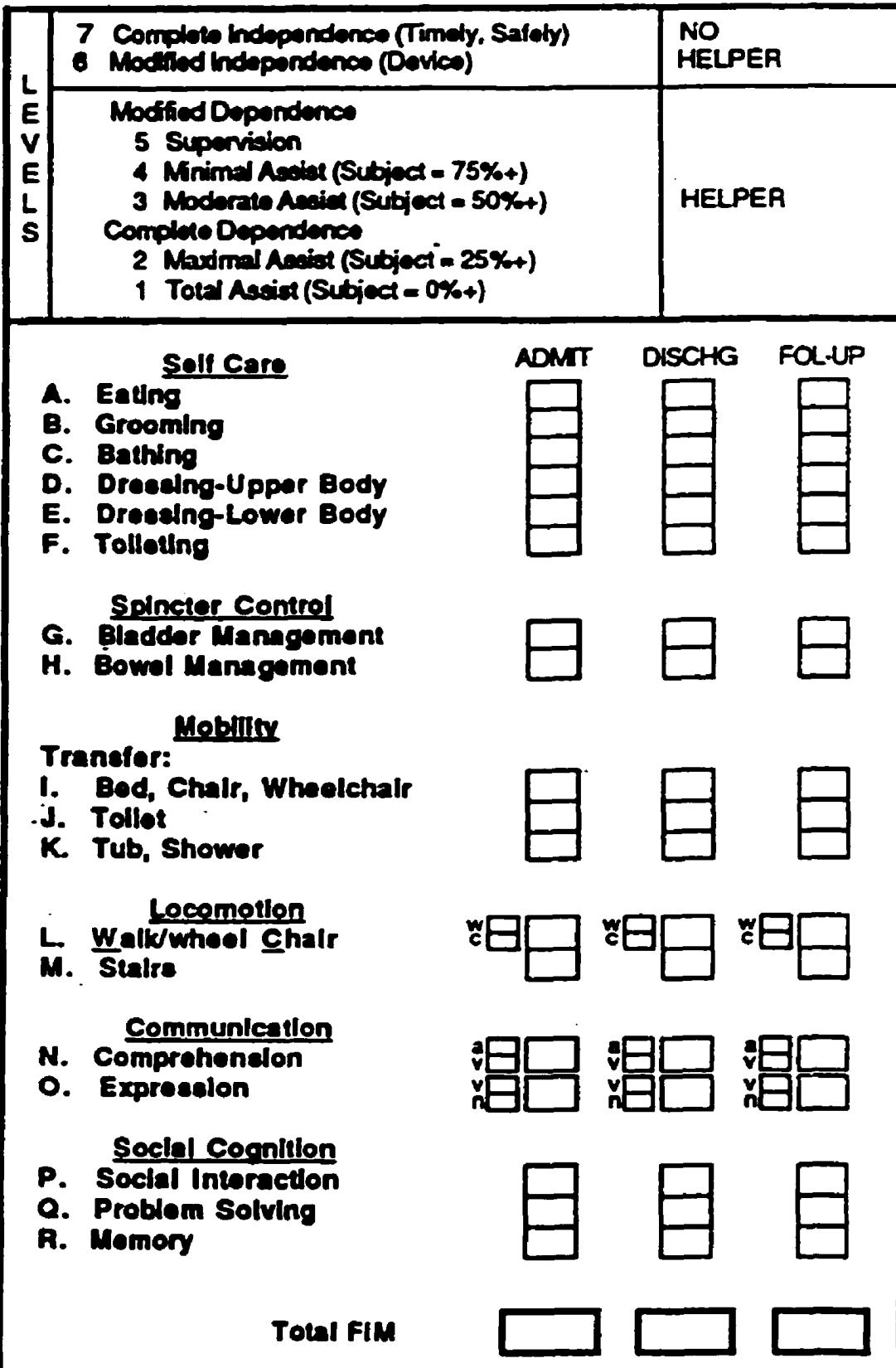

NOTE: Loave no blanks; enter 1 if patient not tostable due to riak.

Copyright 1990 Research Foundation - State University of New York

COPY FREELY - BUT DO NOT CHANGE 


\section{APPENDIX 6}

\section{SPITZER QUALITY OF LIFE INDEX}

Reprinted by permission of the publisher from

(Spitzer WO, Dobson AJ, Hall J, et al. Measuring the quality of life of cancer patients)

Journal of Chronic Diseases, Vol. 34, pp. 585-597.

Copyright 1981 by Elsevier Science Inc. 
FATIENT NO.:

EVALUATION FERIOD:

$$
\begin{aligned}
& \text { 1. } 1 \text { Month } \\
& \text { 2. I Months }
\end{aligned}
$$

SOURCE OF INFORMATION:

$$
\begin{aligned}
& \text { 1. Patient } \\
& \text { 2. Caretaker }
\end{aligned}
$$

DATE of eVALLiation
COOEID

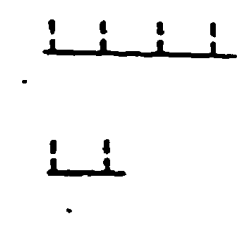

3. 12 Months

EVALP SOURCE

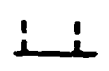

3. Other
$\frac{1}{M M D O Y Y}$

1) On a scale from 1 to 10. how would you rate your own quality of life dur the past weex. Note that 1 = lowest quality and $10=$ highest.

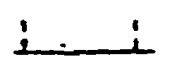

2) Activity: Main activity during the last week:

o. I do not work in any capacity nor do I study nor do I manage my own nousehold

1. I work or study in my usual occupation or manage my own. household or participating in unpaid or voluntary activities. but I need major assistance or a significant reduction in hours worked

2. I work or :study full time, or nearly so, in usual occupation, or manago own household, or participate in voluntary or unpaid activities

3) Daily Living: Ability to look after yourself the last week:

o. I am confined to my home or an institution and cannot manage personal care nor light tasks at all

1. I can travel and perform daily activities only with assistance (another person or special equipment) but can perform lioht tasks

2. I am able to eat, wash, so to the toilet and dress without assistance. I uso public transport or drive my own car

4) Health : Stage of health during the last week:

0 . I feel vory ill or "lousy" most of the time."

1. I lack onergy or only feel "up to par" some of the time.

2. I feel well most of the time 
5) Support.: What support do you receive from others:

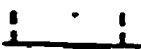

... 0 . The support I receive from family and friends occurs infrequently or only when absolutely necessary.

1. the support I receive from family and friends is 1 imitod.

2. I. have good relationships with others and receive strong support from at least one family member and/or friend

6) Outlook: How do you feel about your life:

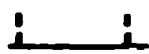

0. I feel frightened and completely confused about things in general

1. I am sometimes troubled because not fully in control of personal life. I am anxious and depressed. at times

2. I am basically a calm person. I generally look forward to things and am able to make own decisions about my 1 ife and

TOTAL SCORE

OLSATOT : i 
APPENDIX 7

\section{CONSENT FORMS}




\section{STROKE RECOVERY: A LONGITUDINAL. CL.INICAL CEREBRAL PERFUSION CORRELATION STUDY}

1. The purpose of this study is to understand the recovery patterns in acute stroke over a one year period.

2. If I agree to participate, the deficits caused by my stroke will be evaluated at certain incervals over one year. They will be correlated with measures of blood flow to different brain regions using a new scanning rechnique called Single Photon Emission Computerized Tomography (SPECT). The tests administered assess perceptual, motor, and language functions and are normal assessment procedures for stroke patients at sunnybrook Health Science Centre. Questionnaires concerning my medical history, mood stace, and abilicy to carry out activities of daily living are also included.

3. In addition, specialized computerized tasks, in which visual stimuli (words, pictures, or symbols) presented on a computer screen, may also be administered. The task is to respond to various tarects by pushing a butcon or sayine a word. During some of these tests, a video camera may be used to monitor my eye movements and my ability to carry out certair motor actions.

4. The time required for each assessinent is 3-4 hours. This can be divided, according to my convenience. into brief sessions. There are no anticipated risks or discomtorts from doing these rasks.

5. These assessments will be carried out in my first week in hospital. and again at one month, three months, and twelve months.

6. I can refuse to participate, and wichdraw from the project at any time without affecting my medical treatment in any way.

7. CT and SPECT scans are usually done as part of the early diagnostic investigation of a person with an acute stroke. These scanning procedures are sometimes repeated, dependine on the circumstances. It is proposed, as part of this study, to repcat my SPECT scan at $1-2$ weeks and one year after my stroke. A SPECT scan is a nuclear medicine technique in which a radioactive tracer is injected into the arm to measure blood flow to different parts of the brain. The scan takes about 30 minutes. This procedure provides additional information about the effects of a stroke on brain function, and involves a radiation exposure of 1.3 Rems. whish is equivalent to two routine brain CT scans. Expressed in every day terms, the lifetime risk of dying from cancer represented by such an exposure, is equivalent to the risk of dying from an accident when driving 1300 miles in a car.

continued... 
8. The information obtained from these assessment and che SPECT scans will be held in confidence but, if agreeable to me. can be made available to the medical team and other caretakers who may be involved in my management. Although I am not guaranteed to benefit from this study, this information could potentially be useful to those involved in my care. and may help me to better understand and deal with my deficits.

9. The neurologist in charge of this study. Dr. Sandra Black (480-4551) will answer any questions I may have at any time.

Witness

Patient's signature 
SURROGATE C.ONSENT FORM

\section{STROKE RECOVERY: A LONGITUDINAL CLINICAL CEIREBIRAL PERFUSION CORRE.L.ATION STUDY}

1. The purpose of this study is to understand the recovery patterns in acute stroke. by carefully evaluating the deficits caused by the stroke at certain intervals over one year. These measures will be correlated with blood flow to different brain regions using a new scanning technique called Single Photon Emission Computerized Tomography (SPECT). The tests administered assess perceptual, motor, and language functions and are llormal assessment procedures for stroke patients at Sunnybrook Health Science Centre. Questionnaires concerning the participant's medical history, mood state, and ability to carry out activities of daily living are also included.

2. In addition, specialized compurerized tasks, in which visual stimuli (words, pictures, or symbols) are presented on a computer screen, may also be administered. The task is to respond to various targets by pushing a button or saying a word. Durine some of these tests, a video camera may be used to monitor the participant's eye movements and ability to carty out. certain moror actions.

4. The time reguired for esch assessment is 3-4 hours. This can be divided, according to the parricipant's convenience, into brief sessions. There are no anticipaled risks or discomforts from doing these tasks.

j. These assessments will be carried out in the participant's first week in hospital, and again at one month, three months, and twelve months.

6. The participant can refuse to participate, and withdraw from the projert at any time without affecting his/her medical treatment in any way.

7. CT and SPECT scans arc usually done as part of the diagnostic investigation of a person with an acute stroke. These scanning procedures are sometimes repeated, depending on the circumstances. It is proposed, as part of this study, to repeat rhe participant's SPECT scan at $1-2$ weeks and one year after his/her stroke. A SPECT scan is a nuclear medicine technique in which a radioactive tracer is injected into the arm to measure blood flow to different parts of the brain. The scan takes about 30 minutes. This procedure provides additional information about the effects of a stroke on brain function, and involves a radiation exposure of 1.3 Rems, which is equivalent to two routine brain CT scans. Expressed in everyday terms. the lifetime risk of dying from cancer represented by such an exposure. is equivalent to the risk of dying from an accident when driving $1300 \mathrm{miles}$ in a car. 
8. The information obtained froin these assessment and the SPECT scans will be held in confidence but, if agreeable to the participant, can be made available to the medical ceam and orher caretakers who may be involved in his/her management. Although the participant is not guaranteed to benefit from this study, this information could potentially be useful to those involved in his/her care, and may help to better understand and deal with deficits caused by the stroke.

9. The neurologist in charge of this study. Dr. Sandra Black (480-4551) will answer any questions the participant or I may have at any ime.

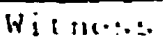

Dace

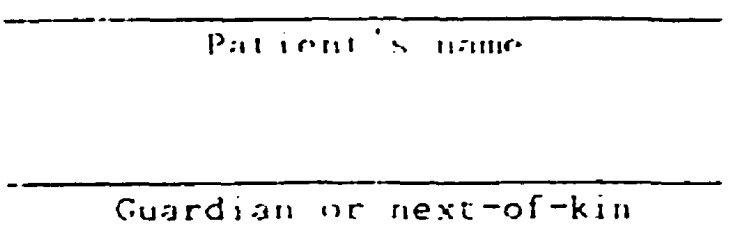

Relatenship to paticat 


\section{APPENDIX 8}

\section{DEMOGRAPHIC PROFILE OF ALL ELIGIBLE SUBJECTS ON ADMISSION}


Profile on Hospital Admission - Categorical Variables All Eligible Subjects ( $n=436$ individuals, 450 strokes)

\begin{tabular}{|c|c|}
\hline Variable & Frequency $(\%)$ \\
\hline $\begin{array}{l}\text { Sex } \\
\text { Male } \\
\text { Female }\end{array}$ & $\begin{array}{l}212(48.6 \%) \\
224(51.4 \%)\end{array}$ \\
\hline $\begin{array}{l}\text { Race } \\
\text { White } \\
\text { Black } \\
\text { Asian } \\
\text { Other } \\
\text { Unknown }\end{array}$ & $\begin{array}{c}404(92.7 \%) \\
11(2.5 \%) \\
15(3.4 \%) \\
5(1.2 \%) \\
1(0.2 \%) \\
\end{array}$ \\
\hline $\begin{array}{l}\text { Marital Status } \\
\text { Single } \\
\text { Married } \\
\text { Widowed } \\
\text { Separated } \\
\text { Divorced } \\
\text { Unknown } \\
\\
\text { Married } \\
\text { Not Married }\end{array}$ & $\begin{array}{c}51(11.7 \%) \\
220(50.5 \%) \\
137(31.4 \%) \\
11(2.5 \%) \\
11(2.5 \%) \\
6(1.4 \%) \\
\\
220(51.2 \%) \\
210(48.8 \%)\end{array}$ \\
\hline $\begin{array}{l}\quad \text { Place of Residence } \\
\text { Home } \\
\text { Structured Retirement } \\
\text { Nursing Home } \\
\text { Skilled Nursing Home } \\
\text { Acute Care of SHSC } \\
\text { Chronic Hospital } \\
\text { Rehabilitation Facility } \\
\text { Unknown } \\
\text { Home } \\
\text { Institution }\end{array}$ & $\begin{array}{c}392(89.9 \%) \\
19(4.4 \%) \\
6(1.4 \%) \\
6(1.4 \%) \\
5(1.2 \%) \\
1(0.2 \%) \\
1(0.2 \%) \\
6(1.4 \%) \\
411(95.6 \%) \\
19(4.4 \%)\end{array}$ \\
\hline
\end{tabular}




\begin{tabular}{|l|c|}
\hline \multicolumn{1}{|c|}{ Variable } & Frequency (\%) \\
\hline \multicolumn{1}{|c|}{ Living Arrangements for those } & \\
Alone & \\
With Family & $122(31.1 \%)$ \\
With Friends & $255(65.1 \%)$ \\
Unknown & $7(1.8 \%)$ \\
& $8(2.0 \% \%)$ \\
Alone & \\
With Others & $122(31.8 \%)$ \\
\hline Vocational Status on Admission & $262(68.2 \%)$ \\
Employed & \\
Homemaker & $50(11.5 \%)$ \\
Student & $63(14.5 \%)$ \\
Unemployed & $1(0.2 \%)$ \\
Retired (age >60 yrs) & $7(1.6 \%)$ \\
Retired for Disability (age $<60$ yrs) & $263(60.3 \%)$ \\
Unknown & $24(5.5 \%)$ \\
\hline \multicolumn{1}{|c|}{ Tyducation } & $28(6.4 \%)$ \\
Primary School & \\
High School & $50(11.5 \%)$ \\
College & $168(38.5 \%)$ \\
University & $38(8.7 \%)$ \\
None & $65(14.9 \%)$ \\
Unknown & $7(1.6 \%)$ \\
\hline & $108(24.8 \%)$ \\
\hline Hemispheric Infarct & \\
Hemispheric Hemorrhage & $10(2.2 \%)$ \\
\hline Bight Hemisphere & \\
Bilateral Lesions & $219(13.8 \%)$ \\
\hline
\end{tabular}




\begin{tabular}{|c|c|}
\hline Variable & Frequency $(\%)$ \\
\hline $\begin{array}{l}\text { Other Illnesses Present } \\
\text { on Admission } \\
\text { Yes } \\
\text { No } \\
\text { Unknown }\end{array}$ & $\begin{array}{c}139(31.9 \%) \\
294(67.4 \%) \\
3(0.7 \%) \\
\end{array}$ \\
\hline $\begin{array}{l}\text { History of Previous Stroke } \\
\text { Yes } \\
\text { No } \\
\text { Unknown }\end{array}$ & $\begin{array}{c}118(27.1 \%) \\
313(71.8 \%) \\
5(1.2 \%) \\
\end{array}$ \\
\hline $\begin{array}{l}\text { History of Diabetes } \\
\text { Yes } \\
\text { No } \\
\text { Unknown }\end{array}$ & $\begin{array}{c}75(17.2 \%) \\
357(81.9 \%) \\
4(0.9 \%) \\
\end{array}$ \\
\hline $\begin{array}{l}\text { History of Cardiovascular } \\
\text { Disease } \\
\text { Yes } \\
\text { No } \\
\text { Unknown }\end{array}$ & $\begin{array}{c}317(72.7 \%) \\
108(24.8 \%) \\
11(2.5 \%) \\
\end{array}$ \\
\hline $\begin{array}{l}\quad \begin{array}{l}\text { History of Musculoskeletal } \\
\text { Disorders }\end{array} \\
\text { Yes } \\
\text { No } \\
\text { Unknown }\end{array}$ & $\begin{array}{c}136(31.2 \%) \\
295(67.7 \%) \\
5(1.1 \%) \\
\end{array}$ \\
\hline
\end{tabular}

Profile on Admission - Continuous Variables All Eligible Subjects ( $\mathrm{n}=436$ individuals)

\begin{tabular}{|l|c|}
\hline \multicolumn{1}{|c|}{ Variable } & Mean ( \pm standard deviation) \\
\hline Age & $74.9( \pm 11.6)$ \\
& (Median $=77$ years) \\
Age (categorical) & Frequency $(\%)$ \\
$<50$ years & $20(4.6 \%)$ \\
$50-75$ years & $172(39.4 \%)$ \\
$>75$ years & $244(56.0 \%)$ \\
\hline
\end{tabular}




\section{APPENDLX 9}

\section{A COMPARISON OF PARTICIPANTS AND NON-PARTICIPANTS}


Profile on Hospital Admission - Categorical Variables A Comparison of Participants ( $n=164)$ and Non-Participants $(n=84)$

\begin{tabular}{|c|c|c|}
\hline Variable & $\begin{array}{c}\text { Participants } \\
\text { Frequency (\%) }\end{array}$ & $\begin{array}{l}\text { Non-Participants } \\
\text { Frequency (\%) }\end{array}$ \\
\hline $\begin{array}{l}\text { Male } \\
\text { Female }\end{array}$ & $\begin{array}{l}91(55.5 \%) \\
73(44.5 \%)\end{array}$ & $\begin{array}{l}42(50.0 \%) \\
42(50.0 \%)\end{array}$ \\
\hline $\begin{array}{l}\text { White } \\
\text { Black } \\
\text { Asian } \\
\text { Other } \\
\end{array}$ & $\begin{array}{c}148(90.2 \%) \\
8(4.9 \%) \\
7(4.3 \%) \\
1(0.6 \%) \\
\end{array}$ & $\begin{array}{c}78(92.9 \%) \\
1(1.2 \%) \\
4(4.8 \%) \\
1(1.2 \%) \\
\end{array}$ \\
\hline $\begin{array}{l}\text { Single Marital Status } \\
\text { Married } \\
\text { Widowed } \\
\text { Separated } \\
\text { Divorced } \\
\text { Unknown } \\
\text { Married } \\
\text { Not Married } \\
\end{array}$ & $\begin{array}{c}20(12.2 \%) \\
90(54.9 \%) \\
47(28.7 \%) \\
2(1.2 \%) \\
5(3.0 \%) \\
- \\
90(54.9 \%) \\
74(45.1 \%) \\
\end{array}$ & $\begin{array}{c}11(13.1 \%) \\
38(45.2 \%) \\
27(32.1 \%) \\
3(3.6 \%) \\
2(2.4 \%) \\
3(3.6 \%) \\
\\
38(46.9 \%) \\
43(53.1 \%) \\
\end{array}$ \\
\hline $\begin{array}{l}\quad \text { Place of Residence } \\
\text { Home } \\
\text { Structured Retirement } \\
\text { Nursing Home } \\
\text { Acute Care of SHSC } \\
\text { Unknown } \\
\text { Home } \\
\text { Institution }\end{array}$ & $\begin{array}{c}157(95.7 \%) \\
4(2.4 \%) \\
- \\
2(1.2 \%) \\
1(0.6 \%) \\
\\
161(98.8 \%) \\
2(1.2 \%)\end{array}$ & $\begin{array}{c}78(92.9 \%) \\
3(3.6 \%) \\
1(1.2 \%) \\
1(1.2 \%) \\
1(1.2 \%) \\
81(97.6 \%) \\
2(2.4 \%)\end{array}$ \\
\hline
\end{tabular}




\begin{tabular}{|c|c|c|}
\hline Variable & $\begin{array}{c}\text { Participants } \\
\text { Frequency (\%) }\end{array}$ & $\begin{array}{c}\text { Non-Participants } \\
\text { Frequency }(\%) \\
\end{array}$ \\
\hline $\begin{array}{l}\text { Living Arrangements for those } \\
\text { Living at Home } \\
\text { Alone } \\
\text { With Family } \\
\text { With Friends } \\
\text { Unknown } \\
\text { Alone } \\
\text { With Others }\end{array}$ & $\begin{array}{c}(n=161) \\
53(32.9 \%) \\
100(62.1 \%) \\
4(2.5 \%) \\
4(2.5 \%) \\
53(33.8 \%) \\
104(66.2 \%) \\
\end{array}$ & $\begin{array}{c}(n=81) \\
25(30.9 \%) \\
51(63.0 \%) \\
1(1.2 \%) \\
4(4.9 \%) \\
25(32.5 \%) \\
52(67.5 \%)\end{array}$ \\
\hline $\begin{array}{l}\text { Vocational Status } \\
\text { Employed } \\
\text { Homemaker } \\
\text { Student } \\
\text { Unemployed } \\
\text { Retired (age }>60 \text { yrs) } \\
\text { Retired for Disability (age }<60 \text { yrs) } \\
\text { Unknown }\end{array}$ & $\begin{array}{c}36(22.0 \%) \\
26(15.8 \%) \\
1(0.6 \%) \\
4(2.4 \%) \\
83(50.6 \%) \\
9(5.5 \%) \\
5(3.0 \%)\end{array}$ & $\begin{array}{c}10(11.9 \%) \\
14(16.7 \%) \\
- \\
- \\
46(54.8 \%) \\
7(8.3 \%) \\
4(4.8 \%)\end{array}$ \\
\hline $\begin{array}{l}\quad \text { Education } \\
\text { Primary School } \\
\text { High School } \\
\text { College } \\
\text { University } \\
\text { None } \\
\text { Unknown }\end{array}$ & $\begin{array}{c}17(10.4 \%) \\
83(50.6 \%) \\
16(9.8 \%) \\
36(22.0 \%) \\
2(1.2 \%) \\
10(6.1 \%)\end{array}$ & $\begin{array}{c}14(16.7 \%) \\
37(44.0 \%) \\
8(9.5 \%) \\
9(10.7 \%) \\
3(3.6 \%) \\
13(15.5 \%)\end{array}$ \\
\hline $\begin{array}{l}\quad \text { Type of Stroke } \\
\text { Hemispheric Infarct } \\
\text { Hemispheric Hemorrhage }\end{array}$ & $\begin{array}{c}144(87.8 \%) \\
20(12.2 \%) \\
\end{array}$ & $\begin{array}{c}81(96.4 \%)^{*} \\
3(3.6 \%)\end{array}$ \\
\hline $\begin{array}{l}\quad \text { Side of Stroke } \\
\text { Left Hemisphere } \\
\text { Right Hemisphere } \\
\text { Bilateral Lesions } \\
\end{array}$ & $\begin{array}{c}71(43.3 \%) \\
89(54.3 \%) \\
4(2.4 \%) \\
\end{array}$ & $\begin{array}{c}35(41.7 \%) \\
48(57.1 \%) \\
1(1.2 \%) \\
\end{array}$ \\
\hline $\begin{array}{l}\text { Other Illnesses Present } \\
\text { on Admission } \\
\text { Yes } \\
\text { No } \\
\text { Unknown }\end{array}$ & $\begin{array}{c}37(22.5 \%) \\
126(76.8 \%) \\
1(0.7 \%)\end{array}$ & $\begin{array}{c}27(32.1 \%) \\
56(66.7 \%) \\
1(1.2 \%)\end{array}$ \\
\hline
\end{tabular}




\begin{tabular}{|l|c|c|}
\hline \multicolumn{1}{|c|}{ Variable } & $\begin{array}{c}\text { Participants } \\
\text { Frequency (\%) }\end{array}$ & $\begin{array}{c}\text { Non-Participants } \\
\text { Frequency (\%) }\end{array}$ \\
\hline Hes & $34(20.7 \%)$ & $15(17.9 \%)$ \\
No & $130(79.3 \%)$ & $68(80.9 \%)$ \\
Unknown & - & $1(1.2 \%)$ \\
\hline History of Diabetes & & \\
Yes & $19(11.6 \%)$ & $21(25.0 \%) \#$ \\
No & $144(87.8 \%)$ & $62(73.8 \%)$ \\
Unknown & $1(0.6 \%)$ & $1(1.2 \%)$ \\
\hline History of Cardiovascular Disease & $118(72.0 \%)$ & $61(72.6 \%)$ \\
Yes & $44(26.8 \%)$ & $22(26.2 \%)$ \\
No & $2(1.2 \%)$ & $1(1.2 \%)$ \\
Unknown & $14(8.5 \%)$ & $6(7.1 \%)$ \\
\hline History of Cancer & $146(89.0 \%)$ & $76(90.5 \%)$ \\
Yes & $4(2.4 \%)$ & $2(2.4 \%)$ \\
No & & \\
Unknown & & $23(27.4 \%)$ \\
\hline \multicolumn{1}{|c|}{ History of Musculoskeletal } & $51(31.1 \%)$ & $60(71.4 \%)$ \\
Yes Disorders & $111(67.7 \%)$ & $1(1.2 \%)$ \\
No & $2(1.2 \%)$ & \\
Unknown & & \\
\hline
\end{tabular}

Note: $X^{2}$ comparisons were performed excluding the unknown category

${ }^{*} \mathrm{p}<0.05$

$\# \mathrm{p}<0.001$ 
Profile on Hospital Admission - Continuous Variables A Comparison of Participants $(n=164)$ and Non-Participants $(n=84)$

\begin{tabular}{|c|c|c|}
\hline Variable & $\begin{array}{l}\text { Participants } \\
\text { Mean e S.D. }\end{array}$ & $\begin{array}{c}\text { Non-Participants } \\
\text { Mean } \pm \text { S.D. }\end{array}$ \\
\hline Age & $\begin{array}{c}70.9 \pm 13.2 \\
(\text { Median }=74)\end{array}$ & $\begin{array}{c}75.4 \pm 11.6 \# \\
\text { (Median = 77) }\end{array}$ \\
\hline $\begin{array}{l}\text { Age (categorical) } \\
<50 \text { years } \\
50-75 \text { years } \\
>75 \text { years } \\
\end{array}$ & $\begin{array}{c}\text { Frequency }(\%) \\
16(9.8 \%) \\
73(44.5 \%) \\
75(45.7 \%) \\
\end{array}$ & $\begin{array}{c}\text { Frequency }(\%) \\
2(2.4 \%)^{*} \\
34(40.5 \%) \\
48(57.1 \%) \\
\end{array}$ \\
\hline Pre-Stroke Quality of Life & $8.8 \odot 1.6$ & $8.1 \bullet 1.7^{*}$ \\
\hline
\end{tabular}

Disability Level Prior to Admission (FIM)

A Comparison of Participants $(n=164)$ and Non-Participants $(n=84)$

\begin{tabular}{|l|c|c|}
\hline \multicolumn{1}{|c|}{ FIM Subcomponent } & $\begin{array}{c}\text { Participants } \\
\text { Frequency (\%) }\end{array}$ & $\begin{array}{c}\text { Non-Participants } \\
\text { Frequency (\%) }\end{array}$ \\
\hline \multicolumn{1}{|c|}{ Motor Ability } & & \\
Independent & $156(95.1 \%)$ & $74(88.1 \%)$ \\
Moderately Dependent & $7(4.2 \%)$ & $6(7.1 \%)$ \\
Dependent & $1(0.6 \%)$ & - \\
Unknown & - & $4(4.5 \%)$ \\
\hline \multicolumn{1}{|c|}{ Cognitive Ability } & $155(94.5 \%)$ & \\
Independent & $6(3.6 \%)$ & $75(89.3 \%)$ \\
Moderately Dependent & $2(1.2 \%)$ & $5(5.9 \%)$ \\
Dependent & $1(0.6 \%)$ & - \\
Unknown & $4(4.8 \%)$ \\
\hline
\end{tabular}

Note: $X^{2}$ comparisons were performed excluding the unknown category 
Stroke Severity Scores on Admission (Impairment) A Comparison of Participants $(n=164)$ and Non-Participants $(n=84)$

\begin{tabular}{|c|c|c|}
\hline Severity & $\begin{array}{c}\text { Participants } \\
\text { Frequency (\%) }\end{array}$ & $\begin{array}{c}\text { Non-Participants } \\
\text { Frequency (\%) }\end{array}$ \\
\hline Mild & $64(39.0 \%)$ & $18(21.4 \%)$ \\
\hline Moderate & $48(29.3 \%)$ & $24(28.6 \%)$ \\
\hline Severe & $22(13.4 \%)$ & $6(7.1 \%)$ \\
\hline Not Assessed & $30(18.3 \%)$ & $36(42.8 \%)$ \\
\hline
\end{tabular}

Note: $X^{2}$ comparisons were performed excluding the unknown category 


\section{ATTACHMENTS}

ETHICS APPROVAL PERMISSIONS TO REPRODUCE COPYRIGHT MATERIAL 


\section{IEGYORNNDUTA}

TO: Dr. S. Black

Neurology, A-4

FROM: Eric M. Heslin, Ph.D.

Chair, Research Ethics Committee

DATE: JulY 12,1990

SUBJECT: Stroke Recovery - A Iongitudinal Clinical cerebral Perfusion Correlation study

Your letter dated June 15, 1990, addressing the concerns of the Ethics Committee, has been received.

This study is now ethically approved.

EMM : js

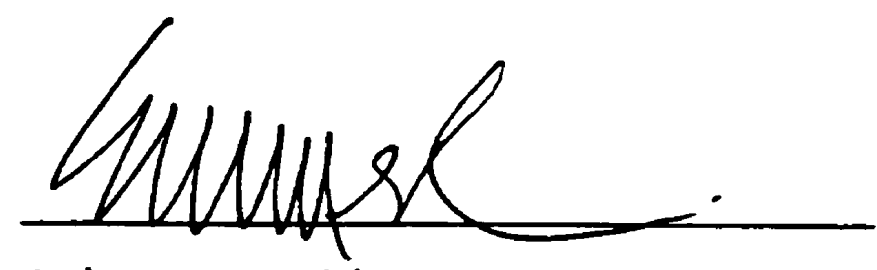

Eric M. Meslin, Ph.D.

Chair, Research Ethics Committee 
34 Ridge Drive Toronto. Ontario M4T 1 B7

Canadian Society for the ICIDH

July 27,1995

P.O. Box 225

Lac St-Charles, Quebec

GOA $2 \mathrm{HO}$

Dear Sir/Madam:

I am completing a masters thesis at the University of Toronto entitled "Post-Stroke Handicap". I am writing to request permission w include the attached material in the thesis and permission for the National Library to make use of the thesis (i.e. to reproduce, loan, distribute or sell copies of the thesis by any means and in any form or format). These rights will in no way restrict republication of the material in any other form by you or by others authorized by you.

The figure to be reprinted is from the August 1991 publication of the ICIDH International Network, Volume 4, Number 3, Page 13 - "Handicaps Creation Process". The ICIDH will be acknowledged as the source of the figure.

If these arrangements meet with your approval, please sign this letter where indicated below and return it to me in the enclosed return envelope.

Thank you for your assistance in this matter.

Yours sincerely,

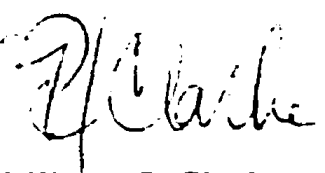

Philippa J. Clarke

M.Sc. Student in Epidemiology

University of Toronto

PERMISSION GRANTED FOR THE USE REQUESTED ABOVE:

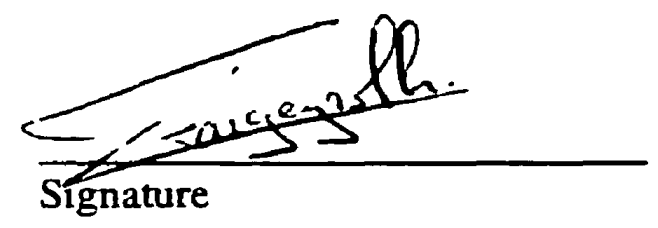

$$
\begin{aligned}
& \text { PATRICK FOUGEYROLLAS Augint i5, } 1995 \\
& \text { Print Name } \\
& \text { CS icis H Changersin/anthot. }
\end{aligned}
$$


34 Ridge Drive

Toronto, Ontario

M4T 187

October 5, 1995

Pergamon Press

Elsevier Science Limited

Bampfylde Street

Exeter EX1 2AH

England

\section{Attention: Permissions Department}

Dear Sir/Madam:

I am completing a masters thesis at the University of Toronto entitled "Handicap in Stroke Survivors". I am writing to request permission to include the following material in the thesis and permission for the National Library to make use of the thesis (i.e. to reproduce, loan, distribute or sell copies of the thesis by any means and in any form or format). These rights will in no way restrict republication of the material in any other form by you or by others authorized by you.

The material to be reprinted is Figure 2 from:

Wood-Dauphinee S, Williams J. Reintegration to Normal Living as a Proxy to Quality of Life. Journal of Chronic Diseases 1987;40(6):491-499. (copy of figure attached)

(now fournal of clinicas Epidemivlogy)

The Journal of Chronic Diseases and Pergamon Press will be acknowledged as the source of the figure.

If these arrangements meet with your approval, please sign this letter where indicated below and return it to me in the enclosed return envelope.

Thank you for your assistance in this matter.

Yours sincerely,

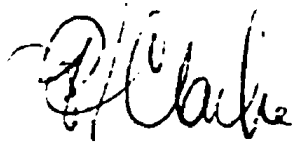

Philippa J. Clarke

M.Sc. Student in Epidemiology

University of Toronto
Permission to reprint as requested is granted by Elsevier Science Inc.,

655 Avenue of the Americas, New York, NY 10010.

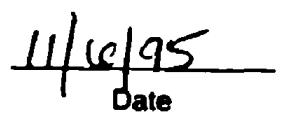

$\frac{\text { Veunica Lepen }}{\text { Simmar }}$

Rights \& Permissions
PERMISSION GRANTED FOR THE USE REQUESTED ABOV

Signature
Print Name
Proper Credit Line:

Reprinted by permission of the publisher from (title and author), name of journal, Vol.

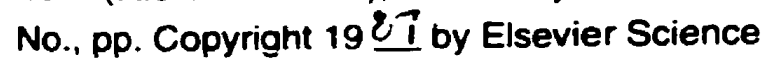
Inc. 
34 Ridge Drive

Toronto. Ontario

M4T 1B7

Permissions Desk

October 5, 1995

Scientific Publishing

American Heart Association

7272 Greenville Avenue

Dallas, Texas

$75231-4596$

Dear Sir/Madam:

I am completing a masters thesis at the University of Toronto entitled "Handicap in Stroke Survivors". I am writing to request permission to include the following material in the thesis and permission for the National Library to make use of the thesis (i.e. to reproduce, loan, distribute or sell copies of the thesis by any means and in any form or format). These rights will in no way restrict republication of the material in any other form by you or by others authorized by you.

The material to be reprinted is the Hemispheric Stroke Scale, Figures 1,2 and 3 from:

Adams RJ, Meador KF, Sethi KD, Grotta JC, Thomson DS. Graded neurologic scale for use in acute hemispheric stroke treatment protocols. Stroke 1987;18:665-669.

(copy of figures attached)

Stroke and the American Heart Association will be acknowledged as the source of the figure.

If these arrangements meet with your approval, please sign this letter where indicated below and return it to me in the enclosed return envelope.

Thank you for your assistance in this matter.

Yours sincerely,

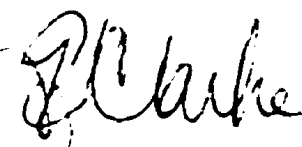

Philippa J. Clarke

M.Sc. Student in Epidemiology

University of Toronto

Permission is grantec provided the publication is credite. Thathe source

PERMISSION GRANTED FOR THE USE REQUESTED ABOVE:

$$
\text { OCT } 101995
$$

AMERICAN HEART ASSUCIATION

Scientific Publications

Signature

Print Name

Departmen:

Date 
Dr. Robert J. Adams

October 26, 1995

Department of Neurology

BIW 338

Medical College of Georgia

112515 th Street

Augusta, Georgia

30912-2366

Dear Dr. Adams:

I am completing a masters thesis at the University of Toronto entitled "Handicap in Stroke Survivors". I am writing to request permission to include the following material in the thesis and permission for the National Library to make use of the thesis (i.e. to reproduce, loan, distribute or sell copies of the thesis by any means and in any form or format). These rights will in no way restrict republication of the material in any other form by you or by others authorized by you. I have already received permission from the Journal in which it was published, but was requested to obtain the author's permission as well.

The material to be reprinted is the Hemispheric Stroke Scale, Figures 1, 2 and 3 from:

Adams RJ, Meador KF, Sethi KD, Grotta JC, Thomson DS. Graded neurologic scale for use in acute hemispheric stroke treatment protocols. Stroke 1987;18:665-669.

If these arrangements meet with your approval, please sign this letter where indicated below and return it to me in the enclosed return envelope.

Thank you for your assistance in this matter.

Yours sincerely,

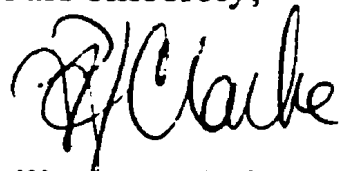

Philippa J. Clarke

M.Sc. Student in Epidemiology

University of Toronto

PERMISSION GRANTED FOR THE USE REQUESTED ABOVE:
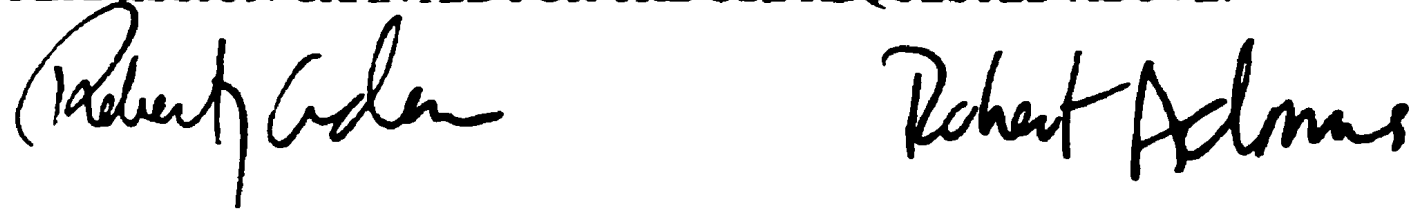

$16 / 6 / 95$

Signature 
Laslo Hunyady

October 5, 1995

Permissions Assistant

Archives of General Psychiatry

515 N. State Street

Chicago, Ilinois

60610

\section{Dear Mr. Hunyady}

I am completing a masters thesis at the University of Toronto entitled "Handicap in Stroke Survivors". I am writing to request permission to include the following material in the thesis and permission for the National Library to make use of the thesis (i.e. to reproduce, loan, distribute or sell copies of the thesis by any means and in any form or format). These rights will in no way restrict republication of the material in any other form by you or by others authorized by you.

The material to be reprinted is Table 3 from:

Zung WWK. A Self-Rating Depression Scale. Archives of General Psychiatry 1965;12:63-70. (copy of table attached)

The Archives of General Psychiatry will be acknowledged as the source of the figure.

If these arrangements meet with your approval, please sign this letter where indicated below and return it to me in the enclosed return envelope.

Thank you for your assistance in this matter.

Yours sincerely,

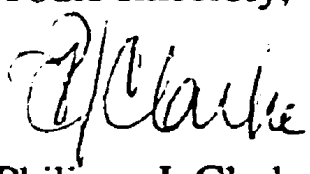

Philippa J. Clarke

M.Sc. Student in Epidemiology

University of Toronto

PERMISSION GRANTED FOR THE USE REQUESTED ABOVE:

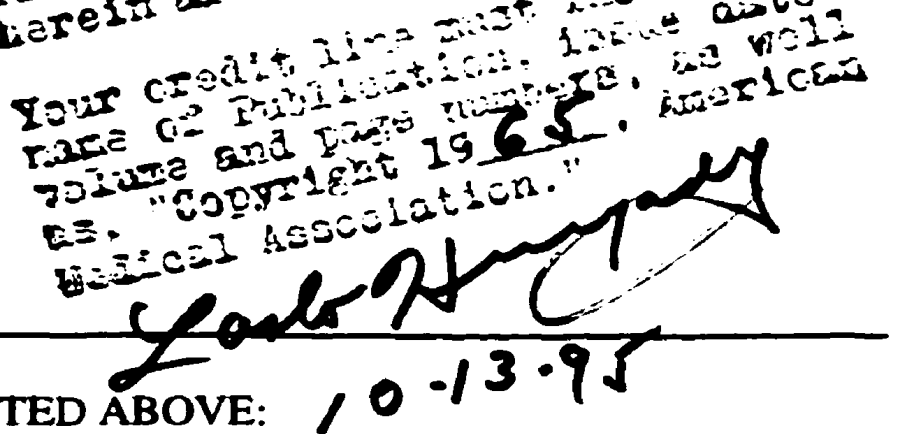

Signature

Print Name

Date 
October 25, 1995

Philippa J. Clarke

34 Ridge Drive

Toronto, Ontario M4T 1B7

Canada

Dear Ms. Clarke,

Thank you for your request for permission to use the Guide for the Uniform Dara Set for Medical Rehabilitation (Adult FIM). Version 4.0 for the purpose of completing your masters degree at the University of Toronto. I understand the project will be entitled "Handicap in Stroke Survivors." Permission is hereby granted to use the Functional Independence Measure (AdultFIM ${ }^{\text {SM) }}$ Version 4.0 as part of your project. Please use the updated form enclosed. When your project is completed, we would be interested in receiving a copy of your results.

Please use the following citation:

Guide for the Uniform Data Set for Medical Rehabilitation (Adult FIM), Version 4.0. Buffalo, NY 14214: State University of New York at Buffalo; 1993.

The following acknowledgment is to be included under each Figure or Table that includes the FIM ${ }^{\text {SM }}$.

Functional Independence Measure, Copyright (C) 1993 Uniform Data System for Medical

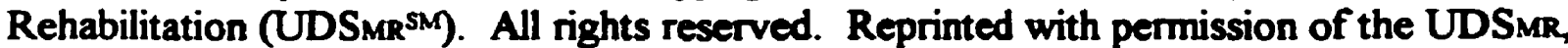
University at Buffalo, 232 Parker Hall, 3435 Main Street, Buffalo, NY 14214.

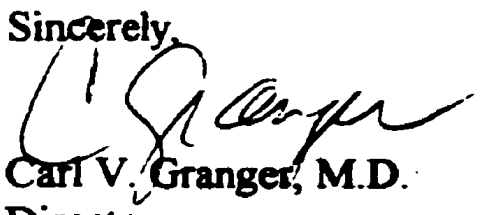

Director

Center for Functional Assessment Research Uniform Data System for Medical Rehabilitation 232 Parker Hall, University at Buffalo, 3435 Main Street. Buffalo, N.Y. 14214-3007 Telephone: (716) 829-2076 • FAX: (716) 829-2080 - E-mail: fimnet@ ubvms.cc.buffalo.edu

Center for Functional Assessment Research • Department of Rehabilitation Medicine School of Medicine and Biomedical Sciences $\bullet$ State University of New York at Buffalo

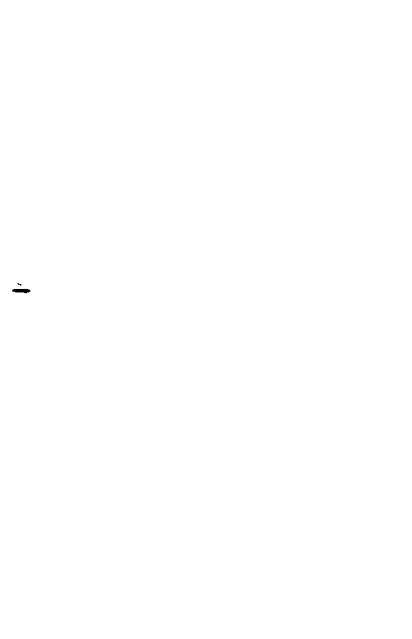


October 5, 1995

Pergamon Press

Elsevier Science Limited

Bampfylde Street

Exeter EX1 2AH

England

\section{Attention: Permissions Department}

Dear Sir/Madam:

I am completing a masters thesis at the University of Toronto entitled "Handicap in Stroke Survivors". I am writing to request permission to include the following material in the thesis and permission for the National Library to make use of the thesis (i.e. to reproduce, loan, distribute or sell copies of the thesis by any means and in any form or format). These rights will in no way restrict republication of the material in any other form by you or by others authorized by you.

The material to be reprinted is Figure 2 from:

Spitzer WO, Dobson AJ, Hall J, Chesterman E, et al. Measuring the quality of life of cancer patients. Journal of Chronic Diseases 1981;34:585-597. (copy of figure attached)

(now fournal of Clinical Epidemiology)

The Joumal of Chronic Diseases and Pergamon Press will be acknowledged as the source of the figure.

If these arrangements meet with your approval, please sign this letter where indicated below and return it to me in the enclosed return envelope.

Thank you for your assistance in this matter.

Yours sincerely,

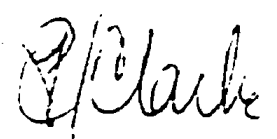

Philippa J. Clarke

M.Sc. Student in Epidemiology

University of Toronto
Permission to reprint as requested is granted by Elsevier Science Inc.,

655 Avenue of the Americas, New York. NY 10010.
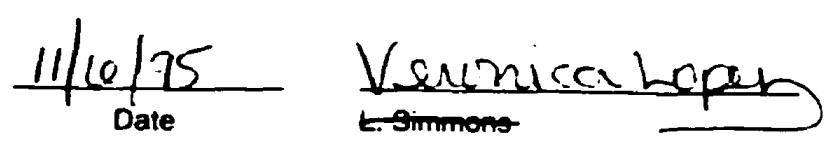

Rights \& Permissions

\section{PERMISSION GRANTED FOR THE USE REQUESTED ABO' Proper Credit Line:}

Signature

Print Name

Reprinted by permission of the publisher from (title and author), name of journal, Vol. No., pp. Copyright $19 \underline{\text { \&l by Elsevier Science }}$ Inc. 